\title{
Lorentz violating $p$-form gauge theories in superspace
}

\author{
Sudhaker Upadhyay ${ }^{1, \mathrm{a}} \mathbb{D}_{\mathrm{D}}$, Mushtaq B. Shah ${ }^{2, \mathrm{~b}}$, Prince A. Ganai ${ }^{2, \mathrm{c}}$ \\ ${ }^{1}$ Centre for Theoretical Studies, Indian Institute of Technology Kharagpur, Kharagpur 721302, India \\ ${ }^{2}$ Department of Physics, National Institute of Technology, Srinagar, Kashmir 190006, India
}

Received: 17 January 2017 / Accepted: 22 February 2017 / Published online: 14 March 2017

(c) The Author(s) 2017. This article is published with open access at Springerlink.com

\begin{abstract}
Very special relativity (VSR) keeps the main features of special relativity but breaks rotational invariance due to an intrinsic preferred direction. We study the VSR-modified extended BRST and anti-BRST symmetry of the Batalin-Vilkovisky (BV) actions corresponding to the $p=1,2,3$-form gauge theories. Within the VSR framework, we discuss the extended BRST invariant and extended BRST and anti-BRST invariant superspace formulations for these BV actions. Here we observe that the VSR-modified extended BRST invariant BV actions corresponding to the $p=1,2,3$-form gauge theories can be written in a manifestly covariant manner in a superspace with one Grassmann coordinate. Moreover, two Grassmann coordinates are required to describe the VSR-modified extended BRST and extended anti-BRST invariant BV actions in a superspace. These results are consistent with the Lorentz-invariant (special relativity) formulation.
\end{abstract}

\section{Overview and motivation}

The standard model, although phenomenologically successful, is unable to explain a variety of issues satisfactorily [1]. The standard model is assumed to be an effective description that works in the low-energy limit of a more fundamental theory (having a quantum description of gravitation also). However, the natural scale for a fundamental theory including gravity is governed by the Planck mass. This leads to an interesting question: whether any aspects of this underlying theory could be revealed through definite experiments with present techniques. To understand this properly, one possibility is to examine proposed fundamental theories for effects that are qualitatively different from standard-model physics. In this regard, one possibility is that the new physics involves

\footnotetext{
a e-mail: sudhakerupadhyay@gmail.com

be-mail: mbstheory72@gmail.com

c e-mail: princeganai@nitsri.net
}

a violation of Lorentz symmetry. In this connection, Cohen and Glashow proposed that the laws of physics need not be invariant under the full Lorentz group but rather under its proper subgroup [2]. An advantage of this hypothesis is that while Lorentz symmetry is violated, the theory still follows the basic postulates of special relativity, like the constancy of the velocity of light. Any scheme with proper Lorentz subgroups along with translations is referred to as very special relativity (VSR).

As an observable consequence of VSR, a novel mechanism for neutrino masses without introducing new particles has been studied in Ref. [3]. A few other observable consequences of VSR have also been given in [4,5]. In recent past, VSR has been studied in various contexts. For instance, the idea of VSR is implemented in de Sitter spacetime where breaking of de Sitter invariance arises in two different ways [6]. Also, it has been shown that the gauge field in the quantum gauge perspective naturally acquires mass without the conventional Higgs mechanism [7]. The modifications due to VSR have also been analyzed for the reducible gauge theories using the BV formulation [8]. Within the VSR framework, the event space underlying the dark matter and the dark gauge fields supports the algebraic structure [9]. The VSR effect in curved spacetimes shows that the SIM(2) symmetry, which leaves the preferred null direction invariant, does not provide the complete couplings to the gravitational background [10]. The proper Lorentz subgroups together with translations are realized in the non-commutative spacetime where the behavior of non-commutativity parameter $\theta^{\mu v}$ is found to be lightlike $[11,12]$. In the VSR scenario, $N=1 \mathrm{SUSY}$ gauge theories contain two conserved supercharges rather than the usual four $[13,14]$. The effects of a quantum correction to VSR is studied to produce a curved spacetime with a cosmological constant [15], where it is shown that the symmetry group ISIM(2) does admit a 2-parameter family of continuous deformations. Recently, a violation of Lorentzinvariance in quantum electrodynamics induced by a very high frequency background wave was studied, where aver- 
aging observables over the rapid field oscillations provides an effective theory [16]. The quantum electrodynamics and the massive spin-1 particle are discussed in VSR in Refs. $[17,18]$. The spontaneous symmetry-breaking mechanism to give a flavor-dependent VSR mass to the gauge bosons is studied in [19]. Interestingly, a quantum field theoretic structure suitable to describe the dark matter suggest that VSR plays the same role for the dark matter fields as special relativity does for the standard-model fields [9].

On the other hands, the supersymmetric version [20] of a non-Abelian gauge theory is a super-geometrical theory of a constrained super 1-form [21]. It is well known that in a superspace formulation there exists a superconnection which is the gauge superfield in a superspace. This, eventually, extends the relation of 1-forms and gauge theories in ordinary spacetime to the superspace. As a result, the entire formalism of differential geometry is valid in the superspace approach. The higher order $p$-forms theories have been studied [21], which contain the gauge superfields characterized by gauge parameters of $(p-1)$-forms. The importance of such a formulation lies in the fact that the well-known formulation of simple supergravity in 11 dimensions [22] explicitly contains a 3-form component gauge field and its extension to superspace $[23,24]$ naturally includes the introduction of a super 3-form gauge superfield. A simple reduction of 11 dimensions to four dimensions then leads to 3-form gauge superfield in the $N=8$ supergravity. Also, antisymmetric tensor fields describe the low-energy excitations in string theories $[25,26]$. The study of higher-form gauge theory is also important for the classical string theories [27], vortex motion in an irrotational, incompressible fluid [28,29], dual formulation of the Abelian Higgs model $[30,31]$ and for supergravity multiplets [32].

To quantize the $p$-form gauge theories, BV formulation is one of the most general and powerful approaches [3339]. One of the important illustrations of this formulation is that it provides a systematic way of accretion of the nontrivial ghost for ghost structure for the case of reducible gauge theories. It has been observed that the antifields of the BV formulation coincide with antighosts of certain collective fields, which ensure that the Schwinger-Dyson equations are satisfied as a consequence of the gauge symmetry algebra $[40,41]$. Also the quantum corrections for anomalous gauge theories can be evaluated from the functional measure as long as a suitable regularization procedure is introduced [42]. A superspace formalism for the Lorentzinvariant BV action of 1-form, 2-form and 3-form gauge theories have been studied [43-47]. The extended BRST and extended anti-BRST invariant formulations (including some shift symmetry) of the Lorentz-invariant BV action have also been studied [43-46,48], which lead to the proper identification of the antifields through the equations of motion of the auxiliary field variables. The importance of shift symme- tries introduced through collective fields lies in the fact that it ensures that the Schwinger-Dyson equations at the level of the BRST algebra can be performed within the Feynman path integral [40]. According to the field redefinition theorem, the particular choice of variables should have no influence on physical quantities like S-matrix elements, which appreciates to formulate the quantization prescription in a more coordinate-independent manner. In Refs. [40,41], the Schwinger-Dyson equations are accomplished in different field variables following the shift symmetry. Although VSR has been studied in various contexts, but the extended BRST and extended anti-BRST invariant formulations with their superspace description remain unstudied in the VSR framework. This provides us an opportunity to bridge this gap.

We consider a non-Abelian 1-form gauge theory in VSR context which remains invariant under a nonlocal (Lorentz breaking) gauge transformation. The equations of motion in VSR-type Lorentz gauge leads a Proca type equation which confirms that non-Abelian vector to have a mass. Although this describes a theory with mass but remains invariant under a (VSR-modified) gauge transformation. This leads to a redundancy in gauge degrees of freedom if we quantize it without fixing a gauge. Therefore, utilizing Faddeev-Popov procedure, we construct an effective action which admits a (VSR modified) BRST transformation. Furthermore, we study the extended BRST symmetry which includes a shift symmetry. This extra symmetry is then gauge fixed (adding new ghosts, antighosts, and auxiliary fields) in such a way that the original action is recovered after the extra fields are integrated out. In order to recover original theory (or to compensate these additional fields), we further introduce antighosts with exactly opposite ghost numbers. Within formulation, these antighosts coincide with the antifields of the $\mathrm{BV}$ formulation analogous to Lorentz-invariant case. We further provide a superspace description of VSR-modified nonAbelian 1-form gauge theory possessing extended BRST symmetry with the help of coordinates $\left(x_{\mu}, \theta\right)$. The superspace description of this theory having extended anti-BRST invariance only needs another fermionic variable $\bar{\theta}$ together with $x_{\mu}$. However, we found that even in VSR-modified theory possessing both the extended BRST and extended antiBRST invariance, one needs a superspace with two grassmann parameters $\theta, \bar{\theta}$ together with $x_{\mu}$ to provide a superfields description. We generalize the results of non-Abelian 1-form gauge theory to the higher-form (for instance 2, 3form) gauge theories also to show consistency of results.

The paper is organized as follows. In Sect. 2, we outline non-Abelian 1-form gauge theory in VSR. Here, we study the VSR modified extended BRST and extended anti-BRST transformations (which include a shift symmetry) for the BV action of the theory. Within this section, we demonstrate a superspace description for the 1-form gauge theory having extended BRST invariance and extended anti-BRST invari- 
ance as the separate cases. Section 3 is devoted to the generalization of results for the non-Abelian 1-form gauge theory to the Abelian 2-form gauge theory. We further generalize these results to the Abelian 3-form gauge theory case in Sect. 4. The paper is summarize with future remarks in the last section. The lengthy calculations are reported in the appendix.

\section{Non-Abelian 1-form gauge theory: VSR-modified BV action in superspace}

In this section, we describe the VSR-modified BV action for non-Abelian 1-form gauge (Yang-Mills) theory in superspace. Let us review first the VSR description of non-Abelian 1-form gauge theory following Ref. [19]. We start by defining the classical Lagrangian density in VSR as follows [19]:

$\mathcal{L}_{0}=-\frac{1}{4} \operatorname{Tr}\left[\tilde{F}^{a \mu \nu} \tilde{F}_{\mu \nu}^{a}\right]$,

where field-strength tensor $\tilde{F}_{\mu \nu}$ is given by

$$
\begin{aligned}
& \tilde{F}_{\mu \nu}=F_{\mu \nu}-\frac{1}{2} m^{2} \\
& \quad \times\left(n_{\nu} \frac{1}{(n \cdot D)^{2}} n^{\alpha} F_{\mu \alpha}-n_{\mu} \frac{1}{(n \cdot D)^{2}} n^{\alpha} F_{\nu \alpha}\right),
\end{aligned}
$$

with

$$
\begin{aligned}
F_{\mu \nu}= & \partial_{\mu} A_{\nu}-\partial_{\nu} A_{\mu}-i\left[A_{\mu}, A_{\nu}\right] \\
& +\frac{1}{2} m^{2} n_{\nu}\left(\frac{1}{(n \cdot \partial)^{2}} \partial_{\mu}(n \cdot A)\right) \\
& -\frac{1}{2} m^{2} n_{\mu}\left(\frac{1}{(n \cdot \partial)^{2}} \partial_{\nu}(n \cdot A)\right) \\
& -\frac{i}{2} m^{2}\left[\frac{1}{(n \cdot \partial)^{2}} n \cdot A,\left(n_{\mu} A_{v}-n_{\nu} A_{\mu}\right)\right] .
\end{aligned}
$$

The vector $n_{\mu}$ is a constant null vector (i.e. $n^{2}=0$ ), which transforms under a VSR transformation so that any term containing ratios involving $n_{\mu}$ are invariant. The covariant derivative is determined by imposing the proper transformation property for the covariant derivative as follows: $D_{\mu}=\partial_{\mu}-i\left[A_{\mu}, \cdot\right]-\frac{i}{2} m^{2} n_{\mu}\left[\left(\frac{1}{(n \cdot \partial)^{2}} n \cdot A\right), \cdot\right]$. We use the following definition to handle the nonlocal terms [19]

$$
\frac{1}{n \cdot D}=\int_{0}^{\infty} \mathrm{d} b \mathrm{e}^{-b n \cdot D}
$$

The equations of motion for the vector field satisfying VSR type Lorentz gauge condition leads to a Proca equation which suggest that vector field has mass [19].
This Lagrangian is also invariant under a VSR-modified (nonlocal) gauge transformation

$$
\begin{aligned}
\delta A_{\mu}= & \partial_{\mu} \lambda-i\left[A_{\mu}, \lambda\right]+\frac{i}{2} m^{2} n_{\mu}\left(\lambda, \frac{1}{(n \cdot \partial)^{2}} n \cdot A\right) \\
& -\frac{1}{2} m^{2} n_{\mu}\left(\frac{1}{(n \cdot \partial)} \lambda\right) \\
& +\frac{i}{2} m^{2} n_{\mu}\left(\frac{1}{(n \cdot \partial)^{2}} n \cdot[A, \lambda]\right),
\end{aligned}
$$

where $\lambda$ is an infinitesimal parameter. Being a (VSR modified) gauge invariant, the non-Abelian 1-form gauge theory contains redundant degrees of freedom. To quantize the theory correctly we need to choose a gauge appropriately. In this context, the gauge-fixed Lagrangian density together with the ghost term is given by

$$
\begin{aligned}
\mathcal{L}_{g f}= & \operatorname{Tr}\left[B \partial^{\mu} A_{\mu}-B \frac{m^{2}}{n \cdot \partial} n^{\mu} A_{\mu}+i \tilde{C}\left(\square-m^{2}\right) C\right. \\
& +\tilde{C} \partial_{\mu}\left[A^{\mu}, C\right]+\frac{m^{2}}{2} \tilde{C}\left(\frac{1}{n \cdot \partial}[n \cdot A, C]\right) \\
& \left.-\frac{m^{2}}{2} \tilde{C}\left(n \cdot \partial\left[C, \frac{n \cdot A}{(n \cdot \partial)^{2}}\right]\right)\right],
\end{aligned}
$$

where $B^{a}$ is an auxiliary field. Here we note that, in comparison to VSR-modified covariant gauges, the gauge-fixed Lagrangian density takes simplest form [8] in VSR-modified light-cone gauge, $\eta \cdot A=0$, where $\eta_{\mu}$ is an arbitrary constant vector that defines a preferred axis in space. Now, the effective quantum Lagrangian density for Yang-Mills theory in VSR is given by

$\mathcal{L}=\mathcal{L}_{0}+\mathcal{L}_{g f}$

This Lagrangian density leads to the following vector field and ghost propagators, respectively:

$$
\begin{aligned}
\Delta^{\mu \nu} & =\frac{1}{p^{2}+m^{2}}\left[\eta^{\mu \nu}-\left(\frac{\alpha-1}{2 \alpha-1}\right) \frac{1}{p^{2}+m^{2}}\right. \\
\times & \left.\left(p^{\mu} p^{\nu}+\frac{1}{2} m^{2}\left(n^{\mu} p^{\nu}+n^{\nu} p^{\mu}\right) \frac{1}{n \cdot p}+\frac{1}{4} m^{4} \frac{n^{\mu} n^{\nu}}{(n \cdot p)^{2}}\right)\right] \\
\Delta_{g h} & =-\frac{1}{p^{2}+m^{2}} .
\end{aligned}
$$

This implies clearly that both the gauge field and ghost field have same mass $m$, consequently, this mass generation is different from Higgs mechanism. This is matter of calculation only to show that this effective Lagrangian density (2.7) is invariant under the following BRST transformations: 


$$
\begin{aligned}
s_{b} A_{\mu}= & \partial_{\mu} C-\frac{m^{2}}{n \cdot \partial} n_{\mu} C-i\left[A_{\mu}, C\right] \\
& +i \frac{m^{2}}{2}\left[C, \frac{n \cdot A}{(n \cdot \partial)^{2}}\right] \\
& +i \frac{m^{2}}{2} n_{\mu}\left(\frac{1}{(n \cdot \partial)^{2}} n \cdot[A, C]\right),=: \mathcal{D}_{\mu} C, \\
s_{b} C= & i C^{2}, \quad s_{b} \tilde{C}=i B, \quad s_{b} B=0 .
\end{aligned}
$$

This BRST transformations are nilpotent in nature, i.e., $s_{b}^{2}=0$. Since the gauge fixing and ghost part of the effective Lagrangian density is BRST exact, so these can also be expressed in terms of BRST variation of gauge-fixing fermion $(\Psi)$. Thus, The effective Lagrangian density can also be expressed as

$\mathcal{L}=\mathcal{L}_{0}+\operatorname{Tr}\left(s_{b} \Psi\right)$,

where the explicit form of the gauge-fixing fermion $\Psi$ is

$\Psi=-i \tilde{C}\left(\partial^{\mu} A_{\mu}-\frac{1}{2} \frac{m^{2}}{n \cdot \partial} n^{\mu} A_{\mu}\right)$.

One could also check that the effective Lagrangian density (2.7) is also invariant under the another nilpotent transformation where the role of ghost and antighost fields are interchanged. These so-called anti-BRST transformations are

$$
\begin{aligned}
s_{a b} A_{\mu}= & \partial_{\mu} \tilde{C}-\frac{m^{2}}{n \cdot \partial} n_{\mu} \tilde{C}-i\left[A_{\mu}, \tilde{C}\right]+i \frac{m^{2}}{2} \\
& \times\left[\tilde{C}, \frac{n \cdot A}{(n \cdot \partial)^{2}}\right]+i \frac{m^{2}}{2} n_{\mu}\left(\frac{1}{(n \cdot \partial)^{2}} n \cdot[A, \tilde{C}]\right), \\
= & \mathcal{D}_{\mu} \tilde{C}, \\
s_{a b} \tilde{C}= & i \tilde{C}^{2}, \quad s_{a b} C=-i B+i C \tilde{C}, \quad s_{a b} B=-i[B, \tilde{C}] .
\end{aligned}
$$

The gauge-fixing and ghost parts of the Lagrangian density are anit-BRST exact also and can also be described in terms of anti-BRST variation of some another gauge-fixing fermion. Here, we would like to state that the VSR gauge fields are massive with a common mass, however, in nature gauge fields may have different masses due to the spontaneous symmetry breaking in VSR with non-Abelian gauge symmetry Ref. [19]. In such a way, the fields can have the usual mass due to spontaneous symmetry breaking in addition to a flavordependent VSR mass.

\subsection{VSR-modified extended BRST invariant BV action}

Now, there exists an interesting question that if the gauge field in VSR is being displaced as $A_{\mu} \rightarrow A_{\mu}-\bar{A}_{\mu}$ : does the gauge symmetry still remain and moreover, how does this shift symmetry affect the underlying BRST structure. In this context, we shift all the fields (within the VSR framework) from their original value as follows:

$$
\begin{aligned}
& A_{\mu} \longrightarrow A_{\mu}-\bar{A}_{\mu}, \quad C \longrightarrow C-\bar{C}, \\
& \tilde{C} \longrightarrow \tilde{C}-\tilde{\tilde{C}}, \quad B \longrightarrow B-\bar{B} .
\end{aligned}
$$

Under these shifts, the Lagrangian density (2.7) in VSR is modified by

$\overline{\mathcal{L}}=\mathcal{L}\left(A_{\mu}-\bar{A}_{\mu}, C-\bar{C}, \tilde{C}-\overline{\tilde{C}}, B-\bar{B}\right)$.

This Lagrangian density is, of course, invariant under the BRST transformation (2.9) with respect to the shifted fields $A_{\mu}-\bar{A}_{\mu}, C-\bar{C}, \tilde{C}-\overline{\tilde{C}}, B-\bar{B}$. In addition, it is also invariant under the local shift symmetry $s_{b} \phi=\mathcal{R}(x), s_{b} \bar{\phi}=$ $\mathcal{R}(x)$, where collective fields $\phi$ and $\bar{\phi}$ are $\left(A_{\mu}, C, \tilde{C}, B\right)$ and $\left(\bar{A}_{\mu}, \bar{C}, \overline{\tilde{C}}, \bar{B}\right)$ respectively, and $\mathcal{R}(x)$ is the generic notation for the Slavnov variations of the fields $\phi$ and $\bar{\phi}$. This deserves further being gauge fixed and, in turn, leads to an additional BRST symmetry. Together, the BRST symmetries will be referred to as the extended BRST symmetry. The extended BRST symmetry transformations corresponding to Lagrangian density (2.14) in VSR are,

$$
\begin{aligned}
s_{b} A_{\mu} & =\psi_{\mu}, \quad s_{b} \bar{A}_{\mu}=\psi_{\mu}-\mathcal{D}_{\mu}^{(A-\bar{A})}(C-\bar{C}), \\
s_{b} C & =\epsilon, \quad s_{b} \bar{C}=\epsilon-i(C-\bar{C})^{2}, \quad s_{b} \tilde{C}=\tilde{\epsilon}, \\
s_{b} \overline{\tilde{C}} & =\tilde{\epsilon}-i(B-\bar{B}), \quad s_{b} B=\rho, \quad s_{b} \bar{B}=\rho,
\end{aligned}
$$

where

$$
\begin{aligned}
& \mathcal{D}_{\mu}^{(A-\bar{A})}(C-\bar{C})=\partial_{\mu}(C-\bar{C})-\frac{m^{2}}{n \cdot \partial} n_{\mu}(C-\bar{C}) \\
& -i[A-\bar{A}, C-\bar{C}]+i \frac{m^{2}}{2}\left[C-\bar{C}, \frac{n \cdot(A-\bar{A})}{(n \cdot \partial)^{2}}\right] \\
& \quad+i \frac{m^{2}}{2} n_{\mu}\left(\frac{1}{(n \cdot \partial)^{2}} n \cdot[A-\bar{A}, C-\bar{C}]\right) .
\end{aligned}
$$

However, $\psi_{\mu}, \epsilon, \tilde{\epsilon}$ and $\rho$ refer the ghost fields associated with shift symmetry for $A_{\mu}, C, \tilde{C}$ and $B$, respectively. Due to nilpotency property of extended BRST symmetry (2.15), it is evident that the variation of these ghost fields $\psi_{\mu}, \epsilon, \tilde{\epsilon}$ and $\rho$ under extended BRST transformation vanish. Now, in order to make theory unchanged, we need to remove the contribution of these ghosts from the physical states. Thus, we introduce the antifields (antighost) $A_{\mu}^{\star}, C^{\star}, \tilde{C}^{\star}$ and $B^{\star}$ corresponding to each ghost field which compensates the net contribution of these ghosts. The BRST variation of these antifields are defined by

$s_{b} A_{\mu}^{\star}=-\zeta_{\mu}, \quad s_{b} C^{\star}=-\sigma, \quad s_{b} \tilde{C}^{\star}=-\tilde{\sigma}, \quad s_{b} B^{\star}=-\tilde{v}$,

where $\zeta_{\mu}, \sigma, \tilde{\sigma}$ and $\tilde{v}$ are auxiliary fields corresponding to shifted fields $\bar{A} \mu, \bar{C}, \overline{\tilde{C}}$ and $\bar{B}$ and do not change under BRST transformation. 
In order to fix the gauge for shift symmetry, we add the following gauge-fixing term to the VSR quantum action (2.7) (and we call the resulting Lagrangian density the BV action):

$$
\begin{aligned}
\overline{\mathcal{L}}_{g f}= & \operatorname{Tr}\left[-\zeta^{\mu} \bar{A}_{\mu}-A^{\mu \star}\left[\psi_{\mu}-\mathcal{D}_{\mu}^{(A-\bar{A})}(C-\bar{C})\right]\right. \\
& +\sigma \overline{\tilde{C}}-C^{\star}[\tilde{\epsilon}-i(B-\bar{B})] \\
& \left.-\tilde{\sigma} \bar{C}+\tilde{C}^{\star}\left[\epsilon-i(C-\bar{C})^{2}\right]+\tilde{v} \bar{B}+B^{\star} \rho\right] .
\end{aligned}
$$

In this way, all the tilde fields will vanish and we then recover our original theory. We note that this gauge-fixed Lagrangian density, $\overline{\mathcal{L}}_{g f}$, is also invariant under the extended BRST symmetry transformations (2.15).

Now, by integrating out the auxiliary fields $\zeta_{\mu}, \sigma, \tilde{\sigma}$ and $\tilde{v}$, this reads

$$
\begin{aligned}
\overline{\mathcal{L}}_{g f}= & \operatorname{Tr}\left[-A_{\mu}^{\star}\left(\psi^{\mu}-\mathcal{D}^{\mu} C\right)-C^{\star}\left(\tilde{\epsilon}^{a}-i B\right)\right. \\
& \left.+\tilde{C}^{\star}\left(\epsilon-i C^{2}\right)+B^{\star} \rho\right] .
\end{aligned}
$$

Since the gauge-fixed Lagrangian density (2.6) is BRST exact and therefore, once can express this in terms of a general gauge-fixing fermion $\Psi\left(A_{\mu}, \tilde{C}, C, B\right)$ as

$\mathcal{L}_{g f}=s_{b} \Psi=-\frac{\delta \Psi}{\delta A_{\mu}} \psi_{\mu}+\frac{\delta \Psi}{\delta C} \epsilon+\frac{\delta \Psi}{\delta \tilde{C}} \tilde{\epsilon}-\frac{\delta \Psi}{\delta B} \rho$,

In the last term, the BRST transformations (2.15) are utilized. Thus, after integrating out the auxiliary fields we set all the fields associated to the shift symmetry to zero, we have the $\mathrm{BV}$ action as

$$
\begin{aligned}
\mathcal{L}_{\text {eff }}=\mathcal{L}_{0} & +\mathcal{L}_{g f}+\overline{\mathcal{L}}_{g f}, \\
=\mathcal{L} & +\operatorname{Tr}\left[\left(-A_{\mu}^{\star}-\frac{\delta \Psi}{\delta A^{\mu}}\right) \psi^{\mu}\right. \\
& +\left(\tilde{C}^{\star}+\frac{\delta \Psi}{\delta C}\right) \epsilon-\left(C^{\star}-\frac{\delta \Psi}{\delta \tilde{C}}\right) \tilde{\epsilon} \\
& \left.+\left(B^{\star}-v \frac{\delta \Psi}{\delta B}\right) \rho+A_{\mu}^{\star} \mathcal{D}_{\mu} C+i C^{\star} B-i \tilde{C}^{\star} C^{2}\right] .
\end{aligned}
$$

To obtain the identifications on the antifields in VSR, it is sufficient to integrate out the ghosts associated with the shift symmetry

$$
A_{\mu}^{\star}=-\frac{\delta \Psi}{\delta A^{\mu}}, \quad \tilde{C}^{\star}=-\frac{\delta \Psi}{\delta C}, \quad C^{\star}=\frac{\delta \Psi}{\delta \tilde{C}}, \quad B^{\star}=\frac{\delta \Psi}{\delta B} .
$$

However, for the VSR-modified gauge-fixing fermion given in (2.11), we determine the antighost fields as follows:

$$
\begin{aligned}
\bar{A}_{\mu}^{a \star} & =-i \partial_{\mu} \tilde{C}+\frac{i}{2} \frac{m^{2}}{n \cdot \partial} n_{\mu} \tilde{C}, \quad \tilde{C}^{a \star}=0, \\
\bar{C}^{a \star} & =-i \partial_{\mu} A^{a \mu}+\frac{i}{2} \frac{m^{2}}{n \cdot \partial} n_{\mu} A^{a \mu}, \\
B^{a \star} & =0
\end{aligned}
$$

Here we observe that analogous to Lorentz-invariant case, antifields get identification naturally. This clarifies the geometric interpretation of the antifields on the line of MaurerCartan 1-forms. Plugging these antighost fields in (2.21), we can recover the original Lagrangian density of YM theory in VSR.

\subsection{VSR-modified extended BRST invariant superspace formulation}

We know that superspace formulations for gauge theories can be built up in such a manner that the BRST transformations are realized as translations along the Grassmannian coordinate [50,51]. In order to describe the VSR-modified BRST invariant BV action in superspace, we need an extra (Grassmannian) coordinate $\theta$ together with $x^{\mu}$. Superspace formulations for the VSR-modified BRST transformation are obtained by associating with each field a superfield of the form

$$
\begin{aligned}
\mathfrak{A}_{\mu}(x, \theta) & =A_{\mu}(x)+\theta \mathcal{D}_{\mu} C, \\
\mathfrak{C}(x, \theta) & =C(x)+i \theta C^{2}, \\
\tilde{\mathfrak{C}}(x, \theta) & =\tilde{C}(x)+i \theta B .
\end{aligned}
$$

The shifted superfields in VSR will be consistent only if these can be written by

$$
\begin{aligned}
\mathbb{A}_{\mu}(x, \theta) & =\mathfrak{A}_{\mu}(x, \theta)-\overline{\mathfrak{A}}_{\mu}(x, \theta) \\
& =\left(A_{\mu}-\bar{A}_{\mu}\right)+\theta \mathcal{D}_{\mu}^{(A-\bar{A})}(C-\bar{C}), \\
\mathbb{C}(x, \theta) & =\mathfrak{C}(x, \theta)-\overline{\mathfrak{C}}(x, \theta)=(C-\bar{C})+i \theta(C-\bar{C})^{2}, \\
\tilde{\mathbb{C}}(x, \theta) & =\tilde{\mathfrak{C}}(x, \theta)-\overline{\tilde{\mathfrak{C}}}(x, \theta) \\
& =\tilde{C}(x)-\overline{\tilde{C}}(x)+i \theta(B-\tilde{B}) .
\end{aligned}
$$

This, however, does not determine the individual superfields uniquely and there is the arbitrariness. So, to be consistent with above discussion, we can define the original superfields and tilde superfields with the help of extended BRST transformation as follows:

$$
\begin{aligned}
& \mathfrak{A}_{\mu}(x, \theta)=A_{\mu}+\theta \psi_{\mu}, \\
& \overline{\mathfrak{A}}_{\mu}(x, \theta)=\bar{A}_{\mu}+\theta\left(\psi_{\mu}-\mathcal{D}_{\mu}^{(A-\bar{A})}(C-\bar{C})\right),
\end{aligned}
$$




$$
\begin{aligned}
\mathfrak{C}(x, \theta)=C+\theta \epsilon, & \overline{\mathfrak{C}}(x, \theta)=\bar{C}+\theta\left(\epsilon-i(C-\bar{C})^{2}\right), \\
\tilde{\mathfrak{C}}(x, \theta)=\tilde{C}+\theta \bar{\epsilon}, & \overline{\tilde{C}}(x, \theta)=\overline{\tilde{C}}-\theta(\tilde{\epsilon}-i B+i \tilde{B}), \\
\mathfrak{B}(x, \theta)=B+\theta \rho . &
\end{aligned}
$$

Exploiting BRST transformations (2.17), we introduce the super antifields with one grassmannian coordinate in VSR as

$\overline{\mathfrak{A}}_{\mu}^{\star}(x, \theta)=A_{\mu}^{\star}-\theta \zeta_{\mu}$,

$\overline{\mathfrak{C}}^{\star}(x, \theta)=C^{\star}-\theta \sigma$,

$\overline{\tilde{\mathfrak{C}}}^{\star}(x, \theta)=\tilde{C}^{\star}-\theta \tilde{\sigma}$,

$\mathfrak{B}^{\star}(x, \theta)=B^{\star}-\theta \tilde{v}$.

We find that the appropriate combinations of the superfields of (2.26) and (2.27), leads to the gauge-fixed Lagrangian density corresponding to shift symmetry in VSR (2.18) as follows:

$\overline{\mathcal{L}}_{g f}=\operatorname{Tr}\left[\frac{\partial}{\partial \theta}\left(\overline{\mathfrak{A}}_{\mu}^{\star} \mathfrak{A}^{\mu}+\overline{\tilde{\mathfrak{C}}^{\star}} \overline{\mathfrak{C}}-\overline{\tilde{\mathfrak{C}}} \overline{\mathfrak{C}}^{\star}-\mathfrak{B}^{\star} \mathfrak{B}\right)\right]$.

The VSR-modified gauge-fixed fermion (2.11) in extended BRST superspace formulation can be written as

$$
\begin{aligned}
\Omega(x, \theta)= & -i \tilde{C}\left(\partial_{\mu}-\frac{1}{2} \frac{m^{2}}{n \cdot \partial} n_{\mu}\right) A^{\mu} \\
& +i \theta\left[\tilde{C}\left(\partial_{\mu}-\frac{1}{2} \frac{m^{2}}{n \cdot \partial} n_{\mu}\right) \psi^{\mu a}\right. \\
& \left.-\tilde{\epsilon}\left(\partial_{\mu}-\frac{1}{2} \frac{m^{2}}{n \cdot \partial} n_{\mu}\right) A^{\mu}\right] .
\end{aligned}
$$

Here, it is evident that $\theta$ component of the above expression gives the gauge-fixing Lagrangian density corresponding to the original BRST symmetry (2.6), i.e.,

$\mathcal{L}_{g f}=\operatorname{Tr}\left[\frac{\partial}{\partial \theta} \Omega(x, \theta)\right]$

Being the $\theta$ component of a super gauge-fixed fermion, the invariance under the extended BRST transformations is evident.

\subsection{VSR-modified extended anti-BRST symmetry}

In this subsection, we construct the VSR-modified extended anti-BRST transformation. The importance of anti-BRST transformation lies in the fact that while the anti-BRST invariance does not lead to any additional information beyond what BRST invariance provides, it helps put the theory in a proper geometrical setting. The extended anti-BRST transformations which leaves the BV action invariant are

$$
\begin{aligned}
s_{a b} A_{\mu} & =A_{\mu}^{\star}+\mathcal{D}_{\mu}^{(A-\bar{A})}(\tilde{C}-\overline{\tilde{C}}), \quad s_{a b} \bar{A}_{\mu}=A_{\mu}^{\star}, \\
s_{a b} \tilde{C} & =\tilde{C}^{\star}+i(\tilde{C}-\overline{\tilde{C}})^{2}, \quad s_{a b} \overline{\tilde{C}}=\tilde{C}^{\star}, \\
s_{a b} C & =C^{\star}-i B+i \bar{B}+i(C-\bar{C})(\tilde{C}-\overline{\tilde{C}}), \quad s_{a b} \bar{C}=C^{\star}, \\
s_{a b} B & =B^{\star}-i[(B-\bar{B}), \tilde{C}-\overline{\tilde{C}}], \quad s_{a b} \bar{B}=B^{\star}, \\
s_{a b} \psi_{\mu} & =\zeta_{\mu}+\mathcal{D}_{\mu}^{(A-\bar{A})}(B-\bar{B})-\left[\mathcal{D}_{\mu}^{(A-\bar{A})}(C-\bar{C})\right](\tilde{C}-\overline{\tilde{C}}), \\
s_{a b} \epsilon & =\sigma-[B-\bar{B}, C-\bar{C}]+(\tilde{C}-\overline{\tilde{C}})(C-\bar{C})^{2}, \\
s_{a b} \tilde{\epsilon} & =\tilde{\sigma}-[B-\bar{B}, \tilde{C}-\overline{\tilde{C}}], \quad s_{a b} \rho=\tilde{v} .
\end{aligned}
$$

Rest fields, whose anti-BRST transformations are not written here do not change under the extended anti-BRST transformation. To describe the superspace formulation of YangMills theory in VSR having both the extended BRST and extended anti-BRST invariance, we need two extra Grassmannian coordinates $\theta, \bar{\theta}$. It is now straightforward to write the superfields in this formulation where the BRST and antiBRST transformations merely correspond to translations in the $\theta$ and $\bar{\theta}$ coordinates respectively. Thus, we see that the results of superspace description of Lorentz-invariant 1-form theory [46] also hold in the case of Lorentz breaking theory.

\section{2-form gauge theory: VSR-modified BV action in superspace}

The study of Abelian 2-form gauge theory is important because it plays a crucial role in studying the theory for classical strings [27], vortex motion in an irrotational, incompressible fluid [28,29] and the dual formulation of the Abelian Higgs model [30]. In this section, we discuss the VSRmodified extended BRST and extended anti-BRST transformations (which include a shift symmetry) for the BV action of 2-form gauge theory. We further demonstrate a superspace description for the 2-form gauge theory having extended BRST invariance and extended anti-BRST invariance. To do so, we start with the classical Lagrangian density for Abelian rank-2 antisymmetric tensor field $\left(B_{\mu \nu}\right)$ theory in VSR as $[7,8]$

$\mathcal{L}_{0}=\frac{1}{12} \tilde{F}_{\mu \nu \rho} \tilde{F}^{\mu \nu \rho}$

where $\tilde{F}_{\mu \nu \rho}$ is the VSR-modified field-strength tensor defined as $\tilde{F}_{\mu \nu \rho} \equiv \partial_{\mu} B_{v \rho}+\partial_{\nu} B_{\rho \mu}+\partial_{\rho} B_{\mu \nu}-\frac{1}{2} \frac{m^{2}}{n \cdot \partial} n_{\mu} B_{v \rho}-$ $\frac{1}{2} \frac{m^{2}}{n \cdot \partial} n_{\nu} B_{\rho \mu}-\frac{1}{2} \frac{m^{2}}{n \cdot \partial} n_{\rho} B_{\mu \nu}$. Here $n_{\mu}$ is a fixed null vector and transforms multiplicatively, as before, under a VSR transformation to ensure the invariance of nonlocal terms.

This field-strength tensor and, consequently, Lagrangian density is not invariant under the Lorentz-invariant gauge transformation $\delta B_{\mu \nu}=\partial_{\mu} \zeta_{\nu}-\partial_{\nu} \zeta_{\mu}$, where $\zeta_{\mu}(x)$ is an arbitrary vector field. Rather, this is invariant under the following 
VSR-modified gauge transformation:

$\delta B_{\mu \nu}=\partial_{\mu} \zeta_{\nu}-\partial_{\nu} \zeta_{\mu}-\frac{1}{2} \frac{m^{2}}{n \cdot \partial} n_{\mu} \zeta_{\nu}+\frac{1}{2} \frac{m^{2}}{n \cdot \partial} n_{\nu} \zeta_{\mu}$

Since the Lagrangian density is invariant under the above nonlocal transformation, hence, to quantize this theory following the BRST technique, it is necessary to introduce two anticommuting vector fields $\rho_{\mu}$ and $\tilde{\rho}_{\mu}$, a commuting vector field $\beta_{\mu}$, two anticommuting scalar fields $\chi$ and $\tilde{\chi}$, and the commuting scalar fields $\sigma, \varphi$ and $\tilde{\sigma}$ [33]. Involving all these fields, the gauge breaking term together with the ghosts is given as [7]

$$
\begin{aligned}
& \mathcal{L}_{g f}=i \tilde{\rho}_{\nu}\left(\partial_{\mu} \partial^{\mu} \rho^{v}-\partial_{\mu} \partial^{v} \rho^{\mu}-m^{2} \rho^{v}\right. \\
& \left.+\frac{1}{2} \frac{m^{2}}{n \cdot \partial} n^{\nu} \partial \cdot \rho+\frac{1}{2} \frac{m^{2}}{n \cdot \partial} \partial^{\nu} n \cdot \rho-\frac{1}{4} \frac{m^{4}}{(n \cdot \partial)^{2}} n^{v} n \cdot \rho\right) \\
& -\tilde{\sigma}\left(\partial_{\mu} \partial^{\mu}-m^{2}\right) \sigma+\beta_{\nu} \partial_{\mu} B^{\mu \nu}-\frac{1}{2} m^{2} \beta_{v} \frac{1}{n \cdot \partial} n_{\mu} B^{\mu \nu} \\
& +\lambda_{1} \beta_{\nu} \beta^{\nu}-\beta_{\nu} \partial^{v} \varphi-i \tilde{\chi} \partial_{\mu} \rho^{\mu}+\frac{i}{2} m^{2} \tilde{\chi} \frac{1}{n \cdot \partial} n_{\mu} \rho^{\mu} \\
& -i \lambda_{2} \tilde{\chi} \chi-i \tilde{\rho}^{\mu} \partial_{\mu} \chi-\frac{i}{2} \frac{m^{2}}{n \cdot \partial} \tilde{\rho}^{\mu} n_{\mu} \chi
\end{aligned}
$$

$k_{1}$ and $k_{2}$ are arbitrary gauge parameters. The ghost and ghost of ghost propagators in momentum space are given, respectively, by [8]

$$
\begin{aligned}
D_{\mu \nu}^{g h}(k) & =-\frac{1}{k^{2}+m^{2}}\left[g_{\mu \nu}+\frac{k_{\mu} k_{\nu}}{m^{2}}\right], \\
D^{g g h}(p) & =-\frac{1}{p^{2}+m^{2}} .
\end{aligned}
$$

These expressions suggest that ghost and ghost of ghost have same mass $m$. Also, these propagators show a large momentum behavior similar to the Lorentz-invariant case. Therefore, the 2-form theory in VSR is a renormalizable theory.

By incorporating the gauge breaking term (3.3), the Lagrangian density in VSR reads effectively

$$
\mathcal{L}=\mathcal{L}_{0}+\mathcal{L}_{g f}
$$

which is invariant under the following nilpotent BRST transformation:

$$
\begin{aligned}
s_{b} B_{\mu \nu} & =\left(\partial_{\mu} \rho_{\nu}-\partial_{\nu} \rho_{\mu}-\frac{1}{2} \frac{m^{2}}{n \cdot \partial} n_{\mu} \rho_{v}+\frac{1}{2} \frac{m^{2}}{n \cdot \partial} n_{\nu} \rho_{\mu}\right), \\
s_{b} \rho_{\mu} & =-i \partial_{\mu} \sigma+\frac{i}{2} \frac{m^{2}}{n \cdot \partial} n_{\mu} \sigma, s_{b} \sigma=0, \quad s_{b} \tilde{\rho}_{\mu}=i \beta_{\mu}, \\
s_{b} \beta_{\mu} & =0, \quad s_{b} \tilde{\sigma}=-\tilde{\chi}, \quad s_{b} \tilde{\chi}=0, \quad s_{b} \varphi=\chi, \quad s_{b} \chi=0 .
\end{aligned}
$$

Since the gauge-fixing and ghost part of the effective Lagrangian density is BRST exact and therefore can be expressed in terms of BRST variation of some gauge-fixed fermion $\Psi$. Therefore,

$\mathcal{L}_{g f}=s_{b} \Psi$

where $\Psi$ has the following form:

$$
\begin{aligned}
\Psi= & -i\left[\tilde{\rho}_{\nu} \partial_{\mu} B^{\mu \nu}+\tilde{\sigma} \partial_{\mu} \rho^{\mu}+\varphi \partial_{\mu} \tilde{\rho}^{\mu}-\tilde{\rho}_{\nu} k_{1} \beta^{\nu}\right. \\
& -\varphi k_{2} \tilde{\chi}-\frac{\tilde{\rho}_{\nu}}{2} \frac{m^{2}}{n \cdot \partial} n_{\mu} B^{\mu \nu} \\
& \left.-\frac{\tilde{\sigma}}{2} \frac{m^{2}}{n \cdot \partial} n_{\mu} \rho^{\mu}-\frac{\varphi}{2} \frac{m^{2}}{n \cdot \partial} n_{\mu} \tilde{\rho}^{\mu}\right] .
\end{aligned}
$$

This gauge-fixed fermion is very important to identify the antifields of the BV action.

\subsection{VSR-modified extended BRST invariant BV action}

The extended BRST and extended anti-BRST invariant formulations of the Lorentz-invariant BV action lead to the proper identification of the antifields through the equations of motion of the auxiliary field variables [43-46,48]. The study of extended (including shift) symmetries introduced through collective fields is important because it ensures that the Schwinger-Dyson equations at the level of the BRST algebra can be performed within the Feynman path integral [40]. In this subsection, we study the VSR-modified extended BRST invariant BV action. To do so, we first deviate all the fields from their original values. This enlarges in a trivial way the symmetry content of the theory, adding extra shift symmetries. To study the extended BRST structure of the Abelian rank-2 tensor field theory in VSR, we shift all the fields of the theory from their original values as follows: $B_{\mu \nu}-\bar{B}_{\mu \nu}, \rho_{\mu}-$ $\bar{\rho}_{\mu}, \tilde{\rho}_{\mu}-\overline{\tilde{\rho}}_{\mu}, \sigma_{\mu}-\bar{\sigma}_{\mu}, \tilde{\sigma}_{\mu}-\overline{\tilde{\sigma}}_{\mu}, \beta_{\mu}-\bar{\beta}_{\mu}, \chi-\bar{\chi}, \tilde{\chi}-\overline{\tilde{\chi}}, \varphi-\bar{\varphi}$. This leads to the following shifted Lagrangian density:

$\overline{\mathcal{L}}=\mathcal{L}_{0}\left(B_{\mu \nu}-\bar{B}_{\mu \nu}\right)+\mathcal{L}_{g f}\left(B_{\mu \nu}-\bar{B}_{\mu \nu}, \Xi-\bar{\Xi}\right)$,

where $\Xi-\bar{\Xi}=\rho_{\mu}-\bar{\rho}_{\mu}, \tilde{\rho}_{\mu}-\overline{\tilde{\rho}}_{\mu}, \sigma_{\mu}-\bar{\sigma}_{\mu}, \tilde{\sigma}_{\mu}-\overline{\tilde{\sigma}}_{\mu}, \beta_{\mu}-$ $\bar{\beta}_{\mu}, \chi-\bar{\chi}, \tilde{\chi}-\overline{\tilde{\chi}}, \varphi-\bar{\varphi}$.

The explicit form of $\mathcal{L}_{g f}\left(B_{\mu \nu}-\bar{B}_{\mu \nu}, \Xi-\bar{\Xi}\right)$ is given by

$$
\begin{aligned}
\mathcal{L}_{g f}= & -i\left[\partial_{\mu} \tilde{\rho}_{\nu} \partial^{\mu} \rho^{v}+m^{2} \tilde{\rho}_{\nu} \rho^{v}-\partial_{\mu} \tilde{\rho}_{\nu} \partial^{\mu} \bar{\rho}^{v}-m^{2} \tilde{\rho}_{\nu} \bar{\rho}^{v}\right. \\
& -\partial_{\mu} \tilde{\rho}_{\nu} \partial^{v} \rho^{\mu}+\frac{1}{2} \partial_{\mu} \tilde{\rho}_{\nu} \frac{m^{2}}{n \cdot \partial} n^{v} \rho^{\mu} \\
& +\partial^{v} \rho^{\mu} \frac{1}{2} \frac{m^{2}}{n \cdot \partial} n_{\mu} \tilde{\rho}_{\nu}-\frac{1}{4}\left(\frac{m^{2}}{n \cdot \partial}\right)^{2} n_{\mu} \tilde{\rho}_{\nu} n^{v} \rho^{\mu} \\
& +\partial_{\mu} \tilde{\rho}_{\nu} \partial^{v} \bar{\rho}^{\mu}-\frac{1}{2} \partial_{\mu} \tilde{\rho}_{\nu} \frac{m^{2}}{n \cdot \partial} n^{v} \bar{\rho}^{\mu} \\
& -\frac{1}{2} \partial^{v} \bar{\rho}^{\mu} \frac{m^{2}}{n \cdot \partial} n_{\mu} \tilde{\rho}_{\nu}+\frac{1}{4}\left(\frac{m^{2}}{n \cdot \partial}\right)^{2} n_{\mu} \tilde{\rho}_{\nu} n^{v} \bar{\rho}^{\mu} \\
& -\partial_{\mu} \overline{\tilde{\rho}}_{\mu} \partial^{\mu} \rho^{\nu}-m^{2} \overline{\tilde{\rho}}_{\nu} \rho^{\nu}+\partial_{\mu} \overline{\tilde{\rho}}_{\nu} \partial^{\mu} \bar{\rho}^{v}
\end{aligned}
$$




$$
\begin{aligned}
& +m^{2} \overline{\tilde{\rho}}_{\nu} \bar{\rho}^{\nu}+\partial_{\mu} \overline{\tilde{\rho}}_{\nu} \partial^{v} \rho^{\mu}-\frac{1}{2} \partial^{\mu} \overline{\tilde{\rho}}^{\mu} \frac{m^{2}}{n \cdot \partial} n^{\nu} \tilde{\rho}^{\mu} \\
& -\frac{1}{2} \partial^{v} \rho^{\mu} \frac{m^{2}}{n \cdot \partial} n_{\mu} \overline{\tilde{\rho}}^{v}+\frac{1}{4}\left(\frac{m^{2}}{n \cdot \partial}\right)^{2} n_{\mu} \overline{\tilde{\rho}}_{\nu} n^{v} \rho^{\mu} \\
& -\partial_{\mu} \overline{\tilde{\rho}}_{\nu} \partial^{v} \bar{\rho}^{\mu}+\frac{1}{2} \partial_{\mu} \overline{\tilde{\rho}}_{\nu} \frac{m^{2}}{n \cdot \partial} n^{v} \bar{\rho}^{\mu}+\frac{1}{2} \partial^{v} \bar{\rho}^{\mu} \frac{m^{2}}{n \cdot \partial} n_{\mu} \overline{\tilde{\rho}}_{\mu} \\
& -\frac{1}{4}\left(\frac{m^{2}}{n \cdot \partial}\right)^{2} n_{\mu} \overline{\tilde{\rho}}_{\nu} n^{\nu} \bar{\rho}^{\mu}+\partial_{\mu} \tilde{\sigma} \partial^{\mu} \sigma \\
& -\partial_{\mu} \tilde{\sigma} \partial^{\mu} \bar{\sigma}-\partial_{\mu} \overline{\tilde{\sigma}} \partial^{\mu} \sigma+\partial_{\mu} \overline{\tilde{\sigma}} \partial^{\mu} \bar{\sigma}+m^{2} \tilde{\sigma} \sigma \\
& -m^{2} \tilde{\sigma} \bar{\sigma}-m^{2} \overline{\tilde{\sigma}} \sigma+m^{2} \overline{\tilde{\sigma}} \bar{\sigma} \\
& +\beta_{\nu} \partial_{\mu} B^{\mu \nu}-\beta_{\nu} \partial_{\mu} \bar{B}^{\mu \nu}+\beta_{\nu} \partial^{\nu} \bar{\varphi}-\bar{\beta}_{\nu} \partial_{\mu} B^{\mu \nu} \\
& -\bar{\beta}_{\nu} \partial_{\mu} \bar{B}^{\mu \nu}+\bar{\beta}_{\nu} \partial^{v} \varphi+\bar{\beta} \partial^{\nu} \bar{\varphi}-\beta_{\nu} \partial^{\nu} \varphi \\
& -\frac{1}{2} \beta_{\nu} \frac{m^{2}}{n \cdot \partial} n_{\mu} B^{\mu \nu}+\frac{1}{2} \beta_{\nu} \frac{m^{2}}{n \cdot \partial} n_{\mu} \bar{B}^{\mu \nu}-\frac{\beta_{v}}{2} \frac{m^{2}}{n \cdot \partial} n^{\nu} \bar{\varphi} \\
& +\frac{\overline{\beta_{v}}}{2} \frac{m^{2}}{n \cdot \partial} n_{\mu} B^{\mu v}+\frac{\overline{\beta_{v}}}{2} \frac{m^{2}}{n \cdot \partial} n_{\mu} B^{\mu \nu} \\
& -\frac{\beta_{v}}{2} \frac{m^{2}}{n \cdot \partial} n^{v} \varphi-\frac{\bar{\beta}_{v}}{2} \frac{m^{2}}{n \cdot \partial} n^{v} \varphi+\frac{\bar{\beta}_{v}}{2} \frac{m^{2}}{n \cdot \partial} n^{v} \bar{\varphi}+k_{1} \beta_{v} \beta^{v} \\
& -k_{1} \beta_{v} \bar{\beta}^{\nu}-k_{1} \bar{\beta}_{v} \beta^{\nu}+k_{1} \bar{\beta}_{v} \bar{\beta}^{v} \\
& -i\left(\tilde{\chi} \partial_{\mu} \rho^{\mu}-\tilde{\chi} \partial_{\mu} \bar{\rho}^{\mu}-\overline{\tilde{\chi}} \partial_{\mu} \rho^{\mu}+\overline{\tilde{\chi}} \partial_{\mu} \bar{\rho}^{\mu}\right. \\
& -\frac{\bar{\chi}}{2} \frac{m^{2}}{n \cdot \partial} n \cdot \rho+\frac{\bar{\chi}}{2} \frac{m^{2}}{n \cdot \partial} n \cdot \bar{\rho}+\frac{\bar{\chi}}{2} \frac{m^{2}}{n \cdot \partial} n \cdot \rho \\
& \left.-\frac{\bar{\chi}}{2} \frac{m^{2}}{n \cdot \partial} n \cdot \bar{\rho}\right)-i\left(\chi \partial_{\mu} \tilde{\rho}^{\mu}-\chi \partial_{\mu} \overline{\tilde{\rho}}^{\mu}-\bar{\chi} \partial_{\mu} \tilde{\rho}^{\mu}\right. \\
& +\bar{\chi} \partial_{\mu} \overline{\tilde{\rho}}^{\mu}-\frac{\chi}{2} \frac{m^{2}}{n \cdot \partial} n \cdot \tilde{\rho}+\frac{\chi}{2} \frac{m^{2}}{n \cdot \partial} n \cdot \overline{\tilde{\rho}} \\
& \left.+\frac{\bar{\chi}}{2} \frac{m^{2}}{n \cdot \partial} n \cdot \tilde{\rho}-\frac{\chi}{2} \frac{m^{2}}{n \cdot \partial} n \cdot \overline{\tilde{\rho}}\right) \\
& \left.-k_{2} \chi \tilde{x}+k_{2} \chi \overline{\tilde{\chi}}+k_{2} \bar{\chi} \tilde{\chi}-k_{2} \bar{\chi} \overline{\tilde{\chi}}\right] \text {. }
\end{aligned}
$$

This Lagrangian density coincides with $\mathcal{L}_{g f}$ of (3.3) when bar fields vanish. It is evident that this Lagrangian density is invariant under the BRST transformation (3.6) for the shifted fields. In addition, there exists the following shift symmetry also:

$s_{b} \Phi(x)=\alpha(x), \quad s_{b} \bar{\Phi}(x)=\alpha(x)$,

which leaves this Lagrangian density invariant. Here $\Phi$ and $\bar{\Phi}$ are generic notation for all fields and shifted fields, respectively. This forms the extended BRST symmetry. The extended BRST transformation, which is comprised by the BRST symmetry along with the above shift symmetry, is then given by

$s_{b} \Phi(x)=\alpha(x), \quad s_{b} \bar{\Phi}(x)=\alpha(x)-\beta(x)$,

where $\beta(x)$ refers to the original BRST transformation collectively, whereas $\alpha(x)$ refers to the shift transformation collectively. In order to quantize the theory collectively, we need to fix the gauge for all the local symmetry. Therefore, corre- sponding to this local shift symmetry, one needs the theory to be gauge fixed and this leads to an additional BRST symmetry [46]. The extended BRST symmetry transformations for all the fields are given by

$$
\begin{aligned}
s_{b} B_{\mu \nu}= & \psi_{\mu \nu}, \quad s_{b} \bar{B}_{\mu \nu}=\psi_{\mu \nu}-\left(\partial_{\mu} \rho_{\nu}-\partial_{\mu} \bar{\rho}_{\nu}-\partial_{\nu} \rho_{\mu}\right. \\
& +\partial_{\nu} \bar{\rho}_{\mu}-\frac{1}{2} \frac{m^{2}}{n \cdot \partial} n_{\mu} \rho_{\nu}+\frac{1}{2} \frac{m^{2}}{n \cdot \partial} n_{\mu} \bar{\rho}_{\nu} \\
& \left.+\frac{1}{2} \frac{m^{2}}{n \cdot \partial} n_{\mu} \bar{\rho}_{\mu}-\frac{1}{2} \frac{m^{2}}{n \cdot \partial} n_{\mu} \bar{\rho}_{\mu}\right), \\
s_{b} \bar{\rho}_{\mu}= & \epsilon_{\mu}+i \partial_{\mu} \sigma-i \partial_{\mu} \bar{\sigma}+\frac{i}{2} \frac{m^{2}}{n \cdot \partial} n_{\mu} \sigma+\frac{i}{2} \frac{m^{2}}{n \cdot \partial} n_{\mu} \bar{\sigma}, \\
s_{b} \tilde{\rho}_{\mu}= & \xi_{\mu}, s_{b} \overline{\tilde{\rho}}_{\mu}=\xi_{\mu}-i \beta_{\mu}+i \bar{\beta}_{\mu}, \quad s_{b} \bar{\sigma}=\varepsilon, \\
s_{b} \sigma= & \varepsilon, s_{b} \beta_{\mu}=\eta_{\mu}, \quad s_{b} \bar{\beta}_{\mu}=\eta_{\mu}, s_{b} \tilde{\sigma}=\psi, \quad s_{b} \tilde{\chi}=\eta, \\
s_{b} \overline{\tilde{\sigma}}= & \psi+\tilde{\chi}-\overline{\tilde{\chi}}, \quad s_{b} \overline{\tilde{\chi}}=\eta, \\
s_{b} \bar{\varphi}= & \phi-\chi+\bar{\chi}, \quad s_{b} \chi=\Sigma, \\
s_{b} \varphi= & \phi, \quad s_{b} \bar{\chi}=\Sigma, s_{b} \xi_{i}=0, \\
\xi_{i} \equiv & {\left[\psi_{\mu \nu}, \epsilon_{\mu}, \xi_{\mu}, \varepsilon, \eta_{\mu}, \psi, \eta, \phi, \Sigma\right] . }
\end{aligned}
$$

The fields $\tilde{\psi}_{\mu \nu}, \epsilon_{\mu}, \xi_{\mu}, \varepsilon, \eta_{\mu}, \psi, \eta, \phi$, and $\Sigma$ are introduced as ghost fields associated with the shift symmetry corresponding to the fields $B_{\mu \nu}, \rho_{\mu}, \tilde{\rho}_{\mu}, \sigma, \beta_{\mu}, \tilde{\sigma}, \tilde{\chi}, \varphi$ and $\chi$, respectively. Further, we add the following antighost fields $B_{\mu \nu}^{\star}, \rho_{\mu}^{\star}, \tilde{\rho}_{\mu}^{\star}, \sigma^{\star}, \tilde{\sigma}^{\star}, \beta_{\mu}^{\star}, \chi^{\star}, \tilde{\chi}^{\star}$ and $\varphi^{\star}$ corresponding to the fields $B_{\mu \nu}, \rho_{\mu}, \tilde{\rho}_{\mu}, \sigma, \beta_{\mu}, \tilde{\sigma}, \tilde{\chi}, \varphi$ and $\chi$, respectively, with opposite statistics. These antighost fields transform under BRST transformations as follows:

$$
\begin{aligned}
& s_{b} B_{\mu \nu}^{\star}=L_{\mu \nu}, \quad s_{b} \rho_{\mu}^{\star}=M_{\mu}, \quad s_{b} \tilde{\rho}_{\mu}^{\star}=\bar{M}_{\mu}, \quad s_{b} \sigma^{\star}=N, \\
& s_{b} \tilde{\sigma}^{\star}=\bar{N}, \quad s_{b} \beta_{\mu}^{\star}=S_{\mu}, \quad s_{b} \chi^{\star}=O, \\
& s_{b} \tilde{\chi}^{\star}=\bar{O}, \quad s_{b} \varphi^{\star}=T,
\end{aligned}
$$

where $L_{\mu \nu}, M_{\mu}, \bar{M}_{\mu}, N, \bar{N}, S_{\mu}, O, \bar{O}, T$ are the NakanishiLautrup type auxiliary fields and do not change under BRST transformation which ensure the nilpotency of BRST symmetry.

Now, we fix the gauge for shift symmetry in VSR by choosing the following gauge-fixed Lagrangian density:

$$
\begin{aligned}
\overline{\mathcal{L}}_{g f}= & L_{\mu \nu} \bar{B}^{\mu \nu}-B_{\mu \nu}^{\star}\left(\psi^{\mu \nu}-\partial^{\mu} \rho^{\nu}+\partial^{\mu} \bar{\rho}^{\nu}+\partial^{v} \rho^{\mu}\right. \\
& -\partial^{\nu} \bar{\rho}^{\mu}+\frac{1}{2} \frac{m^{2}}{n \cdot \partial} n^{\mu} \rho^{\nu}-\frac{1}{2} \frac{m^{2}}{n \cdot \partial} n^{\mu} \bar{\rho}^{\nu} \\
& \left.-\frac{1}{2} \frac{m^{2}}{n \cdot \partial} n^{\nu} \rho^{\mu}+\frac{1}{2} \frac{m^{2}}{n \cdot \partial} n^{\nu} \bar{\rho}^{\mu}\right)+\bar{M}_{\mu} \bar{\rho}^{\mu} \\
& +\tilde{\rho}_{\mu}^{\star}\left(\epsilon^{\mu}+i \partial^{\mu} \sigma-i \partial^{\mu} \bar{\sigma}-i \frac{1}{2} \frac{m^{2}}{n \cdot \partial} n^{\mu} \sigma\right. \\
& \left.+i \frac{1}{2} \frac{m^{2}}{n \cdot \partial} n^{\mu} \bar{\sigma}\right)+M_{\mu} \overline{\tilde{\rho}}^{\mu}+\rho_{\mu}^{\star}\left(\xi^{\mu}-i \beta^{\mu}+i \bar{\beta}^{\mu}\right) \\
& +N \bar{\sigma}-\sigma^{\star} \varepsilon+\bar{N} \overline{\tilde{\sigma}}-\tilde{\sigma}^{\star}(\psi-\tilde{\chi}+\overline{\tilde{\chi}})
\end{aligned}
$$




$$
\begin{aligned}
& +\bar{O} \bar{\chi}+\tilde{\chi}^{\star} \Sigma+O \overline{\tilde{\chi}}+\chi^{\star} \eta+T \bar{\varphi} \\
& -\varphi^{\star}(\phi-\chi+\bar{\chi})+S_{\mu} \bar{\beta}^{\mu}-\beta_{\mu}^{\star} \eta^{\mu},
\end{aligned}
$$

which sets all the bar fields to zero and thus we recover the original theory. This gauge-fixing term is also invariant under the extended BRST symmetry transformations given in Eqs. (3.13) and (3.15). The gauge-fixed extended Lagrangian density, $\overline{\mathcal{L}}_{g f}$, after exploiting the equations of motion of the auxiliary fields, has the following form:

$$
\begin{aligned}
\overline{\mathcal{L}}_{g f}= & -B_{\mu \nu}^{\star}\left(\psi^{\mu \nu}-\partial^{\mu} \rho^{\nu}+\partial^{\nu} \rho^{\mu}+\frac{1}{2} \frac{m^{2}}{n \cdot \partial} n^{\mu} \rho^{\nu}\right. \\
& \left.-\frac{1}{2} \frac{m^{2}}{n \cdot \partial} n^{\nu} \rho^{\mu}\right)+\tilde{\rho}_{\mu}^{\star}\left(\epsilon^{\mu}+i \partial^{\mu} \sigma-\frac{i}{2} \frac{m^{2}}{n \cdot \partial} n^{\mu} \sigma\right) \\
& +\rho_{\mu}^{\star}\left(\xi^{\mu}-i \beta^{\mu}\right)-\sigma^{\star} \epsilon-\tilde{\sigma}^{\star}(\psi-\tilde{\chi}) \\
& +\tilde{\chi}^{\star} \Sigma+\chi^{\star} \eta-\varphi^{\star}(\phi-\chi)-\beta_{\mu}^{\star} \eta^{\mu} .
\end{aligned}
$$

As the gauge-fixed fermion $\Psi$ for Abelian rank-2 antisymmetric tensor field in VSR depends only on the original fields, then the most general gauge-fixed Lagrangian density is given by

$$
\begin{aligned}
& \mathcal{L}_{g f}= s_{b} \Psi=\psi_{\mu \nu} \frac{\delta \Psi}{\delta B_{\mu \nu}}+\epsilon_{\mu} \frac{\delta \Psi}{\delta \rho_{\mu}}+\xi_{\mu} \frac{\delta \Psi}{\delta \tilde{\rho}_{\mu}}+\varepsilon \frac{\delta \Psi}{\delta \sigma} \\
&+\psi \frac{\delta \Psi}{\delta \tilde{\sigma}}+\eta_{\mu} \frac{\delta \Psi}{\delta \beta_{\mu}}+\Sigma \frac{\delta \Psi}{\delta \chi}+\eta \frac{\delta \Psi}{\delta \tilde{\chi}}+\phi \frac{\delta \Psi}{\delta \varphi}
\end{aligned}
$$

Utilizing the fermionic and bosonic behavior of the fields, this can further be written as

$$
\begin{aligned}
\mathcal{L}_{g f}= & -\frac{\delta \Psi}{\delta B_{\mu \nu}} \psi_{\mu \nu}+\frac{\delta \Psi}{\delta \rho_{\mu}} \epsilon_{\mu}+\frac{\delta \Psi}{\delta \tilde{\rho}_{\mu}} \xi_{\mu}-\frac{\delta \Psi}{\delta \sigma} \varepsilon \\
& -\frac{\delta \Psi}{\delta \tilde{\sigma}} \psi-\frac{\delta \Psi}{\delta \beta_{\mu}} \eta_{\mu}+\frac{\delta \Psi}{\delta \chi} \Sigma+\frac{\delta \Psi}{\delta \tilde{\chi}} \eta-\frac{\delta \Psi}{\delta \varphi} \phi .
\end{aligned}
$$

Now, we are able to write the effective Lagrangian density in VSR, $\mathcal{L}_{\text {eff }}=\mathcal{L}_{0}+\mathcal{L}_{g f}+\overline{\mathcal{L}}_{g f}$, as follows:

$$
\begin{aligned}
\mathcal{L}_{\text {eff }}= & \frac{1}{12} \tilde{F}_{\mu \nu \rho} \tilde{F}^{\mu \nu \rho}+B_{\mu \nu}^{\star}\left(\partial^{\mu} \rho^{\nu}-\partial^{\nu} \rho^{\mu}\right. \\
& \left.-\frac{1}{2} \frac{m^{2}}{n \cdot \partial} n^{\mu} \rho^{\nu}+\frac{1}{2} \frac{m^{2}}{n \cdot \partial} n^{\nu} \rho^{\mu}\right) \\
& +i \tilde{\rho}_{\mu}^{\star}\left(\partial^{\mu} \sigma-\frac{1}{2} \frac{m^{2}}{n \cdot \partial} n^{\mu} \sigma\right)-i \rho_{\mu}^{\star} \beta^{\mu} \\
& +\tilde{\sigma}^{\star} \tilde{\chi}+\varphi^{\star} \chi-\left(B_{\mu \nu}^{\star}+\frac{\delta \Psi}{\delta B^{\mu \nu}}\right) \psi^{\mu \nu} \\
& +\left(\rho_{\mu}^{\star}+\frac{\delta \Psi}{\delta \tilde{\rho}^{\mu}}\right) \xi^{\mu}+\left(\tilde{\rho}_{\mu}^{\star}+\frac{\delta \Psi}{\delta \rho^{\mu}}\right) \epsilon^{\mu} \\
& -\left(\sigma^{\star}+\frac{\delta \Psi}{\delta \sigma}\right) \varepsilon-\left(\tilde{\sigma}^{\star}+\frac{\delta \Psi}{\delta \tilde{\sigma}}\right) \psi
\end{aligned}
$$

$$
\begin{aligned}
& +\left(\tilde{\chi}^{\star}+\frac{\delta \Psi}{\delta \chi}\right) \Sigma+\left(\chi^{\star}+\frac{\delta \Psi}{\delta \tilde{\chi}}\right) \eta \\
& -\left(\varphi^{\star}+\frac{\delta \Psi}{\delta \varphi}\right) \phi-\left(\beta_{\mu}^{\star}+\frac{\delta \Psi}{\delta \beta^{\mu}}\right) \eta^{\mu},
\end{aligned}
$$

here Eqs. (3.1), (3.17), and (3.19) are utilized. Exploiting the equations of motion of the ghost fields associated with the shift symmetry, we get the following identifications of the antighost fields:

$$
\begin{aligned}
B_{\mu \nu}^{\star} & =-\frac{\delta \Psi}{\delta B^{\mu \nu}}, \quad \tilde{\rho}_{\mu}^{\star}=-\frac{\delta \Psi}{\delta \rho^{\mu}}, \quad \rho_{\mu}^{\star}=-\frac{\delta \Psi}{\delta \tilde{\rho}^{\mu}}, \\
\sigma^{\star} & =-\frac{\delta \Psi}{\delta \sigma}, \quad \tilde{\sigma}^{\star}=-\frac{\delta \Psi}{\delta \tilde{\sigma}}, \quad \tilde{\chi}^{\star}=-\frac{\delta \Psi}{\delta \chi}, \\
\chi^{\star} & =-\frac{\delta \Psi}{\delta \tilde{\chi}}, \quad \beta_{\mu}^{\star}=-\frac{\delta \Psi}{\delta \beta^{\mu}}, \quad \varphi^{\star}=-\frac{\delta \Psi}{\delta \varphi} .
\end{aligned}
$$

With a particular expression of the gauge-fixed fermion $\Psi$ as given in (3.8), this leads to

$$
\begin{aligned}
B_{\mu \nu}^{\star} & =-i\left(\partial_{\mu}-\frac{1}{2} \frac{m^{2}}{n \cdot \partial} n_{\mu}\right) \tilde{\rho}_{\nu}, \quad \tilde{\rho}_{\mu}^{\star} \\
& =-i\left(\partial_{\mu}-\frac{1}{2} \frac{m^{2}}{n \cdot \partial} n_{\mu}\right) \tilde{\sigma}, \\
\rho_{\mu}^{\star} & =i\left(\partial^{\nu} B_{\nu \mu}-\frac{1}{2} \frac{m^{2}}{n \cdot \partial} n^{\nu} B_{\nu \mu}-k_{1} \beta_{\mu}\right), \quad \sigma^{\star}=0, \\
\tilde{\sigma}^{\star} & =i\left(\partial_{\mu}-\frac{1}{2} \frac{m^{2}}{n \cdot \partial} n_{\mu}\right) \rho^{\mu}, \quad \tilde{\chi}^{\star}=0, \chi^{\star}=-i k_{2} \varphi, \\
\beta_{\mu}^{\star} & =i k_{1} \tilde{\rho}_{\mu}, \quad \tilde{\varphi}^{\star}=i\left(\partial_{\mu} \tilde{\rho}^{\mu}-\frac{1}{2} \frac{m^{2}}{n \cdot \partial} n_{\mu} \tilde{\rho}^{\mu}-k_{2} \tilde{\chi}\right) .
\end{aligned}
$$

Here we see that these antighost fields coincide with the antifields of the theory. We note that these antifields are nonlocal, which describes the features of VSR. Thus, analogous to Lorentz-invariant theory, the antifields are obtained naturally in VSR also. One can see that these identifications lead the effective Lagrangian density to the original form as given in Eq. (3.5). Now, we can describe the gauge-fixing part of the effective Lagrangian density in terms of the BRST variation of a generalized gauge-fixed fermion as follows:

$$
\begin{aligned}
\mathcal{L}_{\text {eff }}= & \mathcal{L}_{0}\left(B_{\mu \nu}-\bar{B}_{\mu \nu}\right) \\
& +s_{b}\left(B_{\mu \nu}^{\star} \bar{B}^{\mu \nu}+\rho_{\mu}^{\star} \overline{\tilde{\rho}}^{\mu}+\tilde{\rho}_{\mu}^{\star} \bar{\rho}^{\mu}+\sigma^{\star} \bar{\sigma}+\tilde{\sigma}^{\star} \overline{\tilde{\sigma}}\right. \\
& \left.+\beta_{\mu}^{\star} \bar{\beta}^{\mu}+\chi^{\star} \overline{\tilde{\chi}}+\tilde{\chi}^{\star} \bar{\chi}+\varphi^{\star} \bar{\varphi}\right), \\
\equiv & \mathcal{L}_{0}\left(B_{\mu \nu}-\bar{B}_{\mu \nu}\right)+s_{b} \Phi^{\star} \bar{\Phi} .
\end{aligned}
$$

Here $\Phi^{\star}$ and $\bar{\Phi}$ are the generic notation for antifields and (corresponding) shifted fields, respectively. The ghost number of $\Phi^{\star} \bar{\Phi}$ is -1 . We thus recover the BV action for Abelian 2 -form gauge theory in VSR with the identification of the antifields. 
3.2 VSR-modified extended BRST invariant superspace formulation

In this section we discuss a superspace formalism of the VSR modified 2-form theory having extended BRST invariance. In this regard, we extend the space to a superspace $\left(x^{\mu}, \theta\right)$ by introducing a fermionic coordinate $\theta$. In this superspace, the "superconnection" 2 -form is defined by

$$
\begin{aligned}
\omega^{(2)}= & \frac{1}{2 !} \mathcal{B}_{\mu \nu}(x, \theta)\left(\mathrm{d} x^{\mu} \wedge \mathrm{d} x^{\nu}\right)+\mathcal{M}_{\mu}(x, \theta)\left(\mathrm{d} x^{\mu} \wedge \mathrm{d} \theta\right) \\
& +\mathcal{N}(x, \theta)(\mathrm{d} \theta \wedge \mathrm{d} \theta),
\end{aligned}
$$

where $d=\mathrm{d} x^{\mu}\left(\partial_{\mu}-\frac{i}{2} \frac{m^{2}}{n \cdot 2} n_{\mu}\right)+\mathrm{d} \theta\left(\partial_{\theta}-\frac{i}{2} \frac{m^{2}}{n \cdot \partial} n_{\theta}\right)$ is the exterior derivative. By requiring the field strength, $F^{(3)}=$ $\mathrm{d} \omega^{(2)}$, to vanish along the $\theta$ direction, we get the following form of the component of the superfields in VSR:

$$
\begin{aligned}
\mathcal{B}_{\mu \nu}(x, \theta) & =B_{\mu \nu}(x)+\theta\left(s_{b} B_{\mu \nu}\right), \\
\mathcal{M}_{\mu}(x, \theta) & =\rho_{\mu}(x)+\theta\left(s_{b} \rho_{\mu}\right), \\
\mathcal{N}(x, \theta) & =\sigma(x)+\theta\left(s_{b} \sigma\right) .
\end{aligned}
$$

In a similar fashion, we are able to write all the fields involved in the extended BV action in superspace as

$$
\begin{aligned}
& \mathcal{B}_{\mu \nu}(x, \theta)=B_{\mu \nu}(x)+\theta \psi_{\mu \nu}, \quad \mathcal{M}_{\mu}(x, \theta)=\rho_{\mu}(x)+\theta \epsilon_{\mu}, \\
& \overline{\mathcal{B}}_{\mu \nu}(x, \theta)=\bar{B}_{\mu \nu}(x)+\theta\left(\psi_{\mu \nu}-\partial_{\mu} \rho_{\nu}\right. \\
& +\partial_{\mu} \bar{\rho}_{\nu}+\partial_{\nu} \rho_{\mu}-\partial_{\nu} \bar{\rho}_{\mu}+\frac{1}{2} \frac{m^{2}}{n \cdot \partial} n_{\mu} \rho_{\nu} \\
& \left.-\frac{1}{2} \frac{m^{2}}{n \cdot \partial} n_{\mu} \bar{\rho}_{\nu}-\frac{1}{2} \frac{m^{2}}{n \cdot \partial} n_{\nu} \rho_{\mu}+\frac{1}{2} \frac{m^{2}}{n \cdot \partial} n_{\nu} \bar{\rho}_{\mu}\right), \\
& \overline{\mathcal{M}}_{\mu}(x, \theta)=\bar{\rho}_{\mu}(x)+\theta\left(\epsilon_{\mu}-i \partial_{\mu} \sigma+i \partial_{\mu} \bar{\sigma}\right. \\
& \left.+\frac{i}{2} \frac{m^{2}}{n \cdot \partial} n_{\mu} \sigma-\frac{i}{2} \frac{m^{2}}{n \cdot \partial} n_{\mu} \bar{\sigma}\right), \\
& \mathcal{N}(x, \theta)=\sigma(x)+\theta \varepsilon, \quad \overline{\mathcal{N}}(x, \theta)=\bar{\sigma}(x)+\theta \varepsilon, \\
& \tilde{\mathcal{M}}_{\mu}(x, \theta)=\tilde{\rho}_{\mu}(x)+\theta \xi_{\mu}, \quad \overline{\tilde{\mathcal{M}}}_{\mu}(x, \theta) \\
& =\overline{\tilde{\rho}}_{\mu}(x)+\theta\left(\xi_{\mu}-i \beta_{\mu}+i \bar{\beta}_{\mu}\right), \\
& \mathcal{S}_{\mu}(x, \theta)=\beta_{\mu}(x)+\theta \eta_{\mu}, \quad \overline{\mathcal{S}}_{\mu}(x, \theta)=\bar{\beta}_{\mu}(x)+\theta \eta_{\mu}, \\
& \tilde{\mathcal{N}}(x, \theta)=\tilde{\sigma}(x)+\theta \psi, \quad \overline{\tilde{\mathcal{N}}}(x, \theta)=\overline{\tilde{\sigma}}(x)+\theta(\psi-\tilde{\chi}+\overline{\tilde{\psi}}), \\
& \mathcal{O}(x, \theta)=\chi(x)+\theta \Sigma, \quad \overline{\mathcal{O}}(x, \theta)=\bar{\chi}(x)+\theta \Sigma, \\
& \tilde{\mathcal{O}}(x, \theta)=\tilde{\chi}(x)+\theta \eta, \quad \overline{\tilde{\mathcal{O}}}(x, \theta)=\overline{\tilde{\chi}}(x)+\theta \eta, \\
& \mathcal{T}(x, \theta)=\varphi(x)+\theta \phi, \quad \overline{\mathcal{T}}(x, \theta)=\bar{\varphi}(x)+\theta(\phi-\chi+\bar{\chi}) .
\end{aligned}
$$

The components of the antifields of the theory in superspace is written

$$
\begin{aligned}
& \overline{\mathcal{B}}_{\mu \nu}^{\star}=B_{\mu \nu}^{\star}+\theta L_{\mu \nu}, \quad \overline{\mathcal{M}}_{\mu}{ }^{\star}=\rho_{\mu}^{\star}+\theta M_{\mu}, \quad \overline{\tilde{\mathcal{M}}}_{\mu}^{\star}=\tilde{\rho}_{\mu}^{\star}+\theta \bar{M}_{\mu}, \\
& \overline{\mathcal{S}}_{\mu}{ }^{\star}=\beta_{\mu}^{\star}+\theta S_{\mu}, \quad \overline{\mathcal{N}}^{\star}=\sigma^{\star}+\theta N, \quad \overline{\tilde{\mathcal{N}}}^{\star}=\tilde{\sigma}^{\star}+\theta \bar{N},
\end{aligned}
$$

$$
\overline{\mathcal{O}}^{\star}=\chi^{\star}+\theta O, \quad \overline{\mathcal{T}}^{\star}=\varphi^{\star}+\theta T, \quad \overline{\tilde{\mathcal{O}}}^{\star}=\tilde{\chi}^{\star}+\theta \bar{O} .
$$

Exploiting expressions (3.27) and (3.28), we derive

$$
\begin{aligned}
\frac{\delta}{\delta \theta} \overline{\mathcal{B}}_{\mu \nu}^{\star} \overline{\mathcal{B}}^{\mu \nu}= & L_{\mu \nu} \bar{B}^{\mu \nu}-B_{\mu \nu}^{\star}\left(\psi^{\mu \nu}-\partial^{\mu} \rho^{\nu}\right. \\
& +\partial^{\mu} \bar{\rho}^{\nu}+\partial^{\nu} \rho^{\mu}-\partial^{\nu} \bar{\rho}^{\mu}+\frac{1}{2} \frac{m^{2}}{n \cdot \partial} n^{\mu} \rho^{\nu} \\
& \left.-\frac{1}{2} \frac{m^{2}}{n \cdot \partial} n^{\mu} \bar{\rho}^{\nu}-\frac{1}{2} \frac{m^{2}}{n \cdot \partial} n^{\nu} \rho^{\mu}+\frac{1}{2} \frac{m^{2}}{n \cdot \partial} n^{\nu} \bar{\rho}^{\mu}\right), \\
\frac{\delta}{\delta \theta} \overline{\tilde{\mathcal{M}}}_{\mu}^{\star} \overline{\mathcal{M}}^{\mu}= & \bar{M}_{\mu} \bar{\rho}^{\mu}+\tilde{\rho}_{\mu}^{\star}\left(\epsilon^{\mu}+i \partial^{\mu} \sigma-i \partial^{\mu} \bar{\sigma}\right. \\
& \left.-\frac{i}{2} \frac{m^{2}}{n \cdot \partial} n^{\mu} \sigma+\frac{i}{2} \frac{m^{2}}{n \cdot \partial} n^{\mu} \bar{\sigma}\right), \\
\frac{\delta}{\delta \theta} \overline{\mathcal{M}}_{\mu} \overline{\mathcal{M}}^{\mu \star}= & M_{\mu} \overline{\tilde{\rho}}^{\mu}+\rho_{\mu}^{\star}\left(\xi^{\mu}-i \beta^{\mu}+i \bar{\beta}^{\mu}\right), \\
\frac{\delta}{\delta \theta} \overline{\mathcal{N}}^{\star} \overline{\mathcal{N}}= & N \bar{\sigma}-\sigma^{\star} \varepsilon, \frac{\delta}{\delta \theta} \overline{\tilde{\mathcal{N}}}^{\star} \overline{\tilde{\mathcal{N}}} \\
= & \bar{N} \overline{\tilde{\sigma}}-\tilde{\sigma}^{\star}(\psi-\tilde{\chi}+\overline{\tilde{\chi}}), \\
\frac{\delta}{\delta \theta} \overline{\tilde{\mathcal{O}}}^{\star} \overline{\mathcal{O}}= & \bar{O} \bar{\chi}+\tilde{\chi}^{\star} \Sigma, \frac{\delta}{\delta \theta} \overline{\tilde{\mathcal{O}}} \overline{\mathcal{O}}^{\star} \\
= & O \overline{\tilde{\chi}}+\chi^{\star} \eta, \\
\frac{\delta}{\delta \theta} \overline{\mathcal{T}}^{\star} \overline{\mathcal{T}}= & T \bar{\varphi}-\varphi^{\star}(\phi-\chi+\bar{\chi}), \frac{\delta}{\delta \theta} \overline{\mathcal{S}}_{\mu}^{\star} \overline{\mathcal{S}}^{\mu} \\
= & S_{\mu} \bar{\beta}^{\mu}-\beta_{\mu}^{\star} \eta^{\mu} .
\end{aligned}
$$

Here, we note that the RHS of the sum of the above expressions coincides with the gauge-fixed Lagrangian density corresponding to the shift symmetry (3.16). Thus, the gaugefixed Lagrangian density in superspace can be written as

$$
\begin{aligned}
\overline{\mathcal{L}}_{g f}= & \frac{\delta}{\delta \theta}\left[\overline{\mathcal{B}}_{\mu \nu}^{\star} \overline{\mathcal{B}}^{\mu \nu}+\overline{\tilde{\mathcal{M}}}_{\mu}^{\star} \overline{\mathcal{M}}^{\mu}+\overline{\tilde{\mathcal{M}}}_{\mu} \overline{\mathcal{M}}^{\mu \star}+\overline{\mathcal{N}}^{\star} \overline{\mathcal{N}}\right. \\
& \left.+\overline{\tilde{\mathcal{N}}}^{\star} \overline{\tilde{\mathcal{N}}}+\overline{\tilde{\mathcal{O}}}^{\star} \overline{\mathcal{O}}+\overline{\tilde{\mathcal{O}}} \overline{\mathcal{O}}^{\star}+\overline{\mathcal{T}}^{\star} \overline{\mathcal{T}}+\overline{\mathcal{S}}_{\mu}^{\star} \overline{\mathcal{S}}^{\mu}\right] .
\end{aligned}
$$

Similar to the Lorentz-invariant theory, the invariance of $\overline{\mathcal{L}}_{g f}$ under the extended BRST transformation is evident from the above expression as it belongs to the $\theta$ component of the superfields. As the gauge-fixing fermion depends on the original fields, the component form of fermionic superfield $\Gamma(x, \theta)$ in superspace is defined as

$$
\begin{aligned}
\Gamma(x, \theta)= & \Psi(x)+\theta\left[-\frac{\delta \Psi}{\delta B_{\mu \nu}} \psi_{\mu \nu}+\frac{\delta \Psi}{\delta \rho_{\mu}} \epsilon_{\mu}+\frac{\delta \Psi}{\delta \tilde{\rho}_{\mu}} \xi_{\mu}\right. \\
& -\frac{\delta \Psi}{\delta \sigma} \varepsilon-\frac{\delta \Psi}{\delta \tilde{\sigma}} \psi-\frac{\delta \Psi}{\delta \beta_{\mu}} \eta_{\mu}+\frac{\delta \Psi}{\delta \chi} \Sigma \\
& \left.+\frac{\delta \Psi}{\delta \tilde{\chi}} \eta-\frac{\delta \Psi}{\delta \varphi} \phi\right] .
\end{aligned}
$$


From the above expression, the most general VSR-modified gauge-fixed Lagrangian density $\mathcal{L}_{g f}$ can be described in the superspace by

$$
\mathcal{L}_{g f}=\frac{\delta \Gamma(x, \theta)}{\delta \theta}
$$

Being the $\theta$ component of fermionic superfield, it is evident that $\mathcal{L}_{g f}$ is invariant under the extended BRST transformation. Thus, the VSR-modified effective Lagrangian density in this superspace formalism is identified as

$$
\begin{aligned}
\mathcal{L}_{\text {eff }}= & \mathcal{L}_{0}\left(B_{\mu \nu}-\bar{B}_{\mu \nu}\right)+\frac{\delta}{\delta \theta}\left[\overline{\mathcal{B}}_{\mu \nu}^{\star} \overline{\mathcal{B}}^{\mu \nu}+\overline{\tilde{\mathcal{M}}}_{\mu}^{\star} \overline{\mathcal{M}}^{\mu}\right. \\
& +\overline{\tilde{\mathcal{M}}}_{\mu} \overline{\mathcal{M}}^{\mu \star}+\overline{\mathcal{N}}^{\star} \overline{\mathcal{N}}+\overline{\tilde{\mathcal{N}}}^{\star} \overline{\tilde{\mathcal{N}}}+\overline{\tilde{\mathcal{O}}}^{\star} \overline{\mathcal{O}} \\
& \left.+\overline{\tilde{\mathcal{O}}} \overline{\mathcal{O}}^{\star}+\overline{\mathcal{T}}^{\star} \overline{\mathcal{T}}+\overline{\mathcal{S}}_{\mu}^{\star} \overline{\mathcal{S}}^{\mu}+\Gamma(x, \theta)\right] .
\end{aligned}
$$

In the VSR framework also, we observe that using the equations of motion of the auxiliary fields and ghost fields of the shift symmetry, this effective Lagrangian density reduces to the original BRST invariant Lagrangian density.

\subsection{VSR-modified extended anti-BRST invariant BV action}

In this subsection, we discuss the VSR-modified extended anti-BRST invariant BV action for the Abelian rank-2 antisymmetric tensor field. First of all, let us write the VSRmodified anti-BRST symmetry transformation $\left(s_{a b}\right)$, which leaves the Lagrangian density (3.5) for the 2-form gauge theory invariant, as follows:

$$
\begin{aligned}
s_{a b} B_{\mu \nu}= & \partial_{\mu} \tilde{\rho}_{\nu}-\partial_{\nu} \tilde{\rho}_{\mu}-\frac{1}{2} \frac{m^{2}}{n \cdot \partial} n_{\mu} \tilde{\rho}_{\nu}+\frac{1}{2} \frac{m^{2}}{n \cdot \partial} n_{\nu} \tilde{\rho}_{\mu} \\
s_{a b} \tilde{\rho}_{\mu}= & -i\left(\partial_{\mu}-\frac{i}{2} \frac{m^{2}}{n \cdot \partial} n_{\mu}\right) \tilde{\sigma} \\
& s_{a b} \tilde{\sigma}=0, s_{a b} \rho_{\mu}=-i \beta_{\mu}, \\
s_{a b} \beta_{\mu}= & 0, s_{a b} \sigma=\chi, s_{a b} \chi=0, s_{a b} \varphi=-\tilde{\chi} \\
& s_{a b} \tilde{\chi}=0 .
\end{aligned}
$$

This VSR-modified anti-BRST transformation is nilpotent and plays an important role in defining physical unitarity. However, this transformation does not anticommute with the BRST transformation (3.6) in absolute fashion, i.e. $\left\{s_{b}, s_{a b}\right\} \neq 0$ for some fields. One should not bother for this in real sense as the absolutely anticommutativity can be achieved on ground of Curci-Ferrari (CF) type restriction. This is emphasized in the next section (in the case of Abelian 3 -form gauge theory) in more detail.
The VSR-modified gauge-fixed fermion corresponding to the anti-BRST transformation, $\bar{\Psi}$, is defined by

$$
\begin{aligned}
\bar{\Psi}= & i\left[\rho_{\nu}\left(\partial_{\mu} B^{\mu \nu}-\frac{1}{2} \frac{m^{2}}{n \cdot \partial} n_{\mu} B^{\mu v}+k_{1} \beta^{v}\right)\right. \\
& -\sigma \partial_{\mu} \tilde{\rho}^{\mu}+\sigma \frac{1}{2} \frac{m^{2}}{n \cdot \partial} n_{\mu} \tilde{\rho}^{\mu} \\
& \left.+\varphi\left(\partial_{\mu} \rho^{\mu}-\frac{1}{2} \frac{m^{2}}{n \cdot \partial} n_{\mu} \rho^{\mu}+k_{2} \chi\right)\right]
\end{aligned}
$$

As the gauge-fixing part of the Lagrangian density is antiBRST exact and, thus, can be written in terms of anti-BRST variation of $\bar{\Psi}$ as follows:

$\mathcal{L}_{g f}=s_{a b} \bar{\Psi}$.

To discuss the extended anti-BRST symmetry within the VSR framework, we do follow the same procedure as in the case of BRST transformation. Thus, here we demand that extended anti-BRST operation on $(\Phi-\bar{\Phi})$ should have same structure of the original anti-BRST transformations with shifted fields. This requirement leads to the following VSR-modified extended anti-BRST transformations:

$$
\begin{aligned}
s_{a b} \bar{B}_{\mu \nu}= & B_{\mu \nu}^{\star}, \quad s_{a b} B_{\mu \nu}=B_{\mu \nu}^{\star}+\left(\partial_{\mu} \tilde{\rho}_{\nu}-\partial_{\mu} \overline{\tilde{\rho}}_{\nu}\right. \\
& -\partial_{\nu} \tilde{\rho}_{\mu}+\partial_{\nu} \overline{\tilde{\rho}}_{\mu}-\frac{i}{2} \frac{m^{2}}{n \cdot \partial} n_{\mu} \tilde{\rho}_{\nu}+\frac{i}{2} \frac{m^{2}}{n \cdot \partial} n_{\mu} \overline{\tilde{\rho}}_{\nu} \\
& \left.+\frac{i}{2} \frac{m^{2}}{n \cdot \partial} n_{\nu} \tilde{\rho}_{\mu}-\frac{i}{2} \frac{m^{2}}{n \cdot \partial} n_{\nu} \overline{\tilde{\rho}}_{\mu}\right), \quad s_{a b} \bar{\rho}_{\mu}=\rho_{\mu}^{\star}, \\
s_{a b} \overline{\tilde{\rho}}_{\mu}= & \tilde{\rho}_{\mu}^{\star}, \quad s_{a b} \tilde{\rho}_{\mu}=\tilde{\rho}_{\mu}^{\star}-i \partial_{\mu} \tilde{\sigma}+i \partial_{\mu} \overline{\tilde{\sigma}} \\
& +\frac{i}{2} \frac{m^{2}}{n \cdot \partial} n_{\mu} \sigma-\frac{i}{2} \frac{m^{2}}{n \cdot \partial} n_{\mu} \overline{\tilde{\sigma}}, \\
s_{a b} \rho_{\mu}= & \rho_{\mu}^{\star}-i \beta_{\mu}+i \bar{\beta}_{\mu}, \quad s_{a b} \overline{\tilde{\sigma}}=\tilde{\sigma}^{\star}, \\
s_{a b} \tilde{\sigma}= & \tilde{\sigma}^{\star}, s_{a b} \bar{\beta}_{\mu}=\beta_{\mu}^{\star}, \\
s_{a b} \beta_{\mu}= & \beta_{\mu}^{\star}, s_{a b} \bar{\sigma}=\sigma^{\star}, \quad s_{a b} \sigma=\sigma^{\star}-\chi+\bar{\chi}, s_{a b} \bar{\chi}=\chi^{\star}, \\
s_{a b} \chi= & \chi^{\star}, s_{a b} \bar{\varphi}=\varphi^{\star}, \quad s_{a b} \varphi=\varphi^{\star}-\tilde{\chi}+\overline{\tilde{\chi}}, \\
s_{a b} \overline{\tilde{\chi}}= & \tilde{\chi}^{\star}, s_{a b} \tilde{\chi}=\tilde{\chi}^{\star} .
\end{aligned}
$$

The antifields $B_{\mu \nu}^{\star}, \tilde{\rho}_{\mu}^{\star}, \rho_{\mu}^{\star}, \tilde{\sigma}^{\star}, \beta_{\mu}^{\star}, \psi, \sigma^{\star}, \chi^{\star}, \varphi^{\star}$ and $\tilde{\chi}^{\star}$ do not vary under the extended anti-BRST transformations as the transformations are nilpotent in nature. Moreover, the ghost fields of the shift symmetry transform under VSRmodified extended anti-BRST transformations as follows:

$$
\begin{aligned}
& s_{a b} \psi_{\mu \nu}=L_{\mu \nu}, \quad s_{a b} \epsilon_{\mu}=M_{\mu}, \quad s_{a b} \xi_{\mu}=\bar{M}_{\mu}, \quad s_{a b} \varepsilon=N, \\
& s_{a b} \psi=\bar{N}, s_{a b} \eta_{\mu}=S_{\mu}, s_{a b} \Sigma=O, s_{a b} \eta=\bar{O}, s_{a b} \phi=T, \\
& s_{a b} \bar{M}_{\mu}=O, \quad s_{a b} L_{\mu \nu}=0, \quad s_{a b} M_{\mu}=0, \quad s_{a b} N=0, \\
& s_{a b} \bar{N}=0, s_{a b} S_{\mu}=0, \quad s_{a b} O=0, \quad s_{a b} \bar{O}=0, \quad s_{a b} \bar{T} .
\end{aligned}
$$

The transformations (3.37) and (3.38) together leads to complete extended anti-BRST transformations in the VSR framework, which leave the shifted effective Lagrangian density 
invariant. With the help of these set of extended anti-BRST transformation, it is straightforward to construct the superspace having extra fermion coordinate $\bar{\theta}$ along with $x_{\mu}$.

\subsection{VSR-modified extended BRST and anti-BRST invariant superspace formulation}

In this subsection, we develop a superspace formulation for VSR modified 2-form gauge theory which is manifestly invariant under both extended BRST and extended antiBRST transformations. To define a superspace for such a theory, we need two Grassmannian coordinates, $\theta$ and $\bar{\theta}$, together with $x_{\mu}$. Therefore, the superfields here depend on superspace $\left(x_{\mu}, \theta, \bar{\theta}\right)$. Within the VSR framework, the "super connection" 2-form $\left(\omega^{(2)}\right)$ and the field strength $\left(F^{(3)}\right)$, respectively, are

$$
\begin{aligned}
\omega^{(2)}= & \frac{1}{2 !} \mathcal{B}_{\mu \nu}(x, \theta, \bar{\theta})\left(d x^{\mu} \wedge d x^{\nu}\right) \\
& +\mathcal{M}_{\mu}(x, \theta, \bar{\theta})\left(d x^{\mu} \wedge d \theta\right)+\mathcal{N}(x, \theta, \bar{\theta})(d \theta \wedge d \theta) \\
& +\tilde{\mathcal{M}}_{\mu}(x, \bar{\theta}, \bar{\theta})\left(d x^{\mu} \wedge d \bar{\theta}\right) \\
& +\tilde{\mathcal{N}}(x, \bar{\theta}, \bar{\theta})(d \bar{\theta} \wedge d \bar{\theta})+\mathcal{T}(x, \bar{\theta}, \bar{\theta})(d \theta \wedge d \bar{\theta}),
\end{aligned}
$$

$F^{(3)}=d \omega^{(2)}$.

Here, the exterior derivative $d$ has the following form:

$$
\begin{aligned}
d= & \mathrm{d} x^{\mu}\left(\partial_{\mu}-\frac{1}{2} \frac{m^{2}}{n \cdot \partial} n_{\mu}\right)+\mathrm{d} \theta\left(\partial_{\theta}-\frac{1}{2} \frac{m^{2}}{n \cdot \partial} n_{\theta}\right) \\
& +\mathrm{d} \bar{\theta}\left(\partial_{\bar{\theta}}-\frac{1}{2} \frac{m^{2}}{n \cdot \partial} n_{\bar{\theta}}\right) .
\end{aligned}
$$

The components of the superfields can be computed by requiring the field strength to vanish along the directions of $\theta$ and $\bar{\theta}$. The explicit expressions for these superfields are calculated in (A1).

Exploiting the expressions of the superfields given in (A1), we compute the following expressions:

$$
\begin{aligned}
\frac{1}{2} \frac{\delta}{\delta \bar{\theta}} \frac{\delta}{\delta \theta} \overline{\mathcal{B}}_{\mu \nu} \overline{\mathcal{B}}^{\mu \nu}= & L_{\mu \nu} \bar{B}^{\mu \nu}-B_{\mu \nu}^{\star}\left(\psi^{\mu \nu} \partial^{\mu} \rho^{\nu}+\partial^{\mu} \rho^{\nu}\right. \\
& +\partial^{v} \rho^{\mu}-\partial^{\nu} \bar{\rho}^{\mu}-\psi^{\mu \nu} \frac{1}{2} \frac{m^{2}}{n \cdot \partial} n^{\mu} \rho^{\nu} \\
& \left.-\frac{1}{2} \frac{m^{2}}{n \cdot \partial} n^{\mu} \rho^{\nu}-\frac{1}{2} \frac{m^{2}}{n \cdot \partial} n^{\nu} \rho^{\mu}+\frac{1}{2} \frac{m^{2}}{n \cdot \partial} n^{\nu} \bar{\rho}^{\mu}\right), \\
\frac{\delta}{\delta \bar{\theta}} \frac{\delta}{\delta \theta} \overline{\tilde{\mathcal{M}}}_{\mu} \overline{\mathcal{M}}^{\mu}= & \bar{M}_{\mu} \bar{\rho}^{\mu}+\tilde{\rho}_{\mu}^{\star}\left(\epsilon^{\mu}+i \partial^{\mu} \sigma-i \partial^{\mu} \bar{\sigma}\right. \\
& \left.-\frac{i}{2} \frac{m^{2}}{n \cdot \partial} n^{\mu} \sigma+\frac{i}{2} \frac{m^{2}}{n \cdot \partial} n^{\mu} \bar{\sigma}\right)+M_{\mu} \overline{\tilde{\rho}}^{\mu} \\
& +\rho_{\mu}^{\star}\left(\xi^{\mu}-i \beta^{\mu}+i \bar{\beta}^{\mu}\right), \\
\frac{1}{2} \frac{\delta}{\delta \bar{\theta}} \frac{\delta}{\delta \theta} \overline{\mathcal{N}} \overline{\mathcal{N}}= & N \bar{\sigma}-\sigma^{\star} \varepsilon, \quad \frac{1}{2} \frac{\delta}{\delta \bar{\theta}} \frac{\delta}{\delta \theta} \overline{\tilde{\mathcal{N}}} \overline{\tilde{\mathcal{N}}} \\
= & \bar{N} \overline{\tilde{\sigma}}-\tilde{\sigma}^{\star}(\psi-\tilde{\chi}+\overline{\tilde{\chi}}),
\end{aligned}
$$

$$
\begin{aligned}
\frac{\delta}{\delta \bar{\theta}} \frac{\delta}{\delta \theta} \overline{\tilde{\mathcal{O}}} \overline{\mathcal{O}} & =\bar{O} \bar{\chi}+\tilde{\chi}^{\star} \Sigma+O \overline{\tilde{\chi}}+\chi^{\star} \eta, \\
\frac{1}{2} \frac{\delta}{\delta \bar{\theta}} \frac{\delta}{\delta \theta} \overline{\mathcal{T}} \overline{\mathcal{T}} & =T \bar{\varphi}-\varphi^{\star}(\phi-\chi+\bar{\chi}), \\
\frac{1}{2} \frac{\delta}{\delta \bar{\theta}} \frac{\delta}{\delta \theta} \overline{\mathcal{S}}_{\mu} \overline{\mathcal{S}}^{\mu} & =S_{\mu} \bar{\beta}^{\mu}-\beta_{\mu}^{\star} \eta^{\mu} .
\end{aligned}
$$

By adding all the equations of the above expression side by side, we see, remarkably, that this is nothing but the expression of Lagrangian density, $\overline{\mathcal{L}}_{g f}$, given in Eq. (3.16). Thus, we can write

$$
\begin{aligned}
\overline{\mathcal{L}}_{g f}= & \frac{1}{2} \frac{\delta}{\delta \bar{\theta}} \frac{\delta}{\delta \theta}\left[\overline{\mathcal{B}}_{\mu \nu} \overline{\mathcal{B}}^{\mu \nu}+2 \overline{\tilde{\mathcal{M}}}_{\mu} \overline{\mathcal{M}}^{\mu}\right. \\
& \left.+\overline{\mathcal{N}} \overline{\mathcal{N}}+\overline{\tilde{\mathcal{N}}} \overline{\tilde{\mathcal{N}}}+2 \overline{\tilde{\mathcal{O}}} \overline{\mathcal{O}}+\overline{\mathcal{T}} \overline{\mathcal{T}}+\overline{\mathcal{S}}_{\mu} \overline{\mathcal{S}}^{\mu}\right] .
\end{aligned}
$$

Eventually, we see that $\overline{\mathcal{L}}_{g f}$ is nothing but the $\theta \bar{\theta}$ component of the composite superfields. Therefore, this certifies the invariance of the $\overline{\mathcal{L}}_{g f}$ under both the extended BRST and extended anti-BRST transformations. The component form of the super gauge-fixing fermion in this superspace is given by

$\Gamma(x, \theta, \bar{\theta})=\Psi+\theta s_{b} \Psi+\bar{\theta} s_{a b} \Psi+\theta \bar{\theta} s_{b} s_{a b} \Psi$,

to express the $\mathcal{L}_{g f}$ as $\frac{\delta}{\delta \theta}[\delta(\bar{\theta}) \Gamma(x, \theta, \bar{\theta})]$. The $\theta \bar{\theta}$ component of $\Gamma(x, \theta, \bar{\theta})$ vanishes due to equations of motion in the theories having both BRST and anti-BRST invariance.

Therefore, the effective Lagrangian density (3.24), which is invariant under both the extended BRST and the extended anti-BRST transformations, can be expressed in superspace by

$$
\begin{aligned}
\mathcal{L}_{\text {eff }}= & \mathcal{L}_{0}\left(B_{\mu \nu}-\bar{B}_{\mu \nu}\right)+\frac{1}{2} \frac{\delta}{\delta \bar{\theta}} \frac{\delta}{\delta \theta}\left[\overline{\mathcal{B}}_{\mu \nu} \overline{\mathcal{B}}^{\mu \nu}+2 \overline{\tilde{\mathcal{M}}}_{\mu} \overline{\mathcal{M}}^{\mu}\right. \\
& \left.+\overline{\mathcal{N}} \overline{\mathcal{N}}+\overline{\tilde{\mathcal{N}}} \overline{\tilde{\mathcal{N}}}+2 \overline{\tilde{\mathcal{O}}} \overline{\mathcal{O}}+\overline{\mathcal{T}} \overline{\mathcal{T}}+\overline{\mathcal{S}}_{\mu} \overline{\mathcal{S}}^{\mu}\right] \\
& +\frac{\delta}{\delta \theta}[\delta(\bar{\theta}) \Gamma(x, \theta, \bar{\theta})]
\end{aligned}
$$

Here, we found that the gauge-fixing parts of the effective Lagrangian density is the $\theta$ components of the certain functional. Thus we observe that the VSR-modified 2-form gauge theory in superspace described by two fermionic parameters also show the same structure as the Lorentz-invariant case.

\section{3-form gauge theory: VSR-modified BV action in superspace}

VSR generalization to the tensor field (reducible gauge) theories has also been done using a BV formulation [7,8]. A rigorous construction of quantum field theory in VSR framwork is also studied [49]. In this connection, we would like study the BV action tensor field of rank-3 in superspace. In 
particular, we generalize our previous results for the case of Abelian 3-form gauge theory which is relevant as it plays a crucial role to study the excitations of the quantized versions of strings, superstrings and related extended objects. The classical Lagrangian density for the Abelian 3-form gauge theory in VSR is given by $[7,8]$,

$\mathcal{L}_{0}=\frac{1}{24} \tilde{F}_{\mu \nu \eta \xi} \tilde{F}^{\mu \nu \eta \xi}$

where the 4-form field-strength tensor $\left(\tilde{F}_{\mu \nu \eta \xi}\right)$ has the following form:

$$
\begin{aligned}
\tilde{F}_{\mu \nu \eta \xi}= & \partial_{\mu} B_{\nu \eta \xi}-\partial_{\nu} B_{\eta \xi \mu}+\partial_{\eta} B_{\xi \mu \nu}-\partial_{\xi} B_{\mu \nu \eta} \\
& -\frac{1}{2} \frac{m^{2}}{n \cdot \partial} n_{\mu} B_{v \eta \xi}+\frac{1}{2} \frac{m^{2}}{n \cdot \partial} n_{\nu} B_{\eta \xi \mu} \\
& -\frac{1}{2} \frac{m^{2}}{n \cdot \partial} n_{\eta} B_{\xi \mu \nu}+\frac{1}{2} \frac{m^{2}}{n \cdot \partial} n_{\xi} B_{\mu \nu \eta} .
\end{aligned}
$$

Here $B_{\mu \nu \eta}$ is totally antisymmetric rank-3 tensor gauge field and $n_{\mu}$ is a constant null vector. The Lagrangian density (4.1) is not invariant under standard gauge transformation as the Lorentz-invariance is broken by choosing an specific direction. However, this Lagrangian density is invariant under the following VSR-modified gauge transformation:

$$
\begin{aligned}
\delta B_{\mu \nu \eta}= & \partial_{\mu} \lambda_{\nu \eta}+\partial_{\nu} \lambda_{\eta \mu}+\partial_{\eta} \lambda_{\mu \nu}-\frac{1}{2} \frac{m^{2}}{n \cdot \partial} n_{\mu} \lambda_{\nu \eta} \\
& -\frac{1}{2} \frac{m^{2}}{n \cdot \partial} n_{\nu} \lambda_{\eta \mu}-\frac{1}{2} \frac{m^{2}}{n \cdot \partial} n_{\eta} \lambda_{\mu \nu},
\end{aligned}
$$

where $\lambda_{\mu \nu}$ is an arbitrary antisymmetric parameter. As this is a (VSR-modified) gauge invariant theory, it contains redundant degrees of freedom. From the expression of (4.3), it is evident that the theory is reducible. Therefore, to quantize this theory correctly we need to fix the gauge appropriately. The gauge-fixed Lagrangian density in VSR is calculated by

$$
\begin{aligned}
\mathcal{L}_{g f}^{B}= & \partial_{\mu} B^{\mu \nu \eta} B_{\nu \eta}-\frac{1}{2} \frac{m^{2}}{n \cdot \partial} n^{\mu} B^{\mu \nu \eta} B_{\nu \eta} \\
& +\frac{1}{2} B_{\mu \nu} \tilde{B}^{\mu \nu}+\partial_{\mu} \tilde{c}_{\nu \eta} \partial^{\mu} c^{\nu \eta}+m^{2} \tilde{c}_{\nu \eta} c^{\nu \eta} \\
& +\partial_{\nu} \tilde{c}_{\eta \mu} \partial^{\mu} c^{\nu \eta}-\partial_{\nu} \tilde{c}_{\eta \mu} \frac{1}{2} \frac{m^{2}}{n \cdot \partial} n^{\mu} c^{\nu \eta} \\
& -\partial^{\mu} c^{\nu \eta} \frac{1}{2} \frac{m^{2}}{n \cdot \partial} n_{\nu} \tilde{c}^{\eta \mu}+n_{\nu} \tilde{c}^{\eta \mu} \frac{1}{4} \frac{m^{2}}{n \cdot \partial} n^{\mu} c^{\nu \eta} \\
& +\partial_{\mu} \tilde{c}_{\mu \nu} \partial^{\mu} c^{\nu \eta}-\partial_{\mu} \tilde{c}_{\mu \nu} \frac{1}{2} \frac{m^{2}}{n \cdot \partial} n^{\mu} c^{\nu \eta} \\
& -\partial^{\mu} c^{\nu \eta} \frac{1}{2} \frac{m^{2}}{n \cdot \partial} n_{\eta} \tilde{c}_{\mu \nu}+n^{\eta} \tilde{c}^{\mu \nu} \frac{1}{4} \frac{m^{2}}{n \cdot \partial} n_{\eta} \tilde{c}_{\mu \nu} \\
& -\partial_{\mu} \tilde{\beta}_{\nu} \partial^{\mu} \beta^{\nu}-m^{2} \tilde{\beta}_{\nu} \beta^{\nu}+\partial_{\nu} \tilde{\beta}_{\mu} \partial^{\mu} \beta^{\nu} \\
& -\partial_{\nu} \tilde{\beta}_{\mu} \frac{1}{2} \frac{m^{2}}{n \cdot \partial} n^{\mu} \beta^{\nu}-\partial^{\mu} \beta^{\nu} \frac{1}{2} \frac{m^{2}}{n \cdot \partial} n_{\nu} \tilde{\beta}_{\mu} \\
& +\frac{1}{4} \frac{m^{2}}{n \cdot \partial} n_{\nu} \tilde{\beta}_{\mu} n^{\mu} \beta^{\nu}-B B_{2}-\frac{1}{2} B_{1}^{2}+\partial_{\mu} \tilde{c}^{\mu \nu} f_{\nu}
\end{aligned}
$$

$$
\begin{aligned}
& -\partial_{\mu} c^{\mu v} \tilde{F}_{v}-\frac{1}{2} \frac{m^{2}}{n \cdot \partial} n_{\mu} \tilde{c}^{\mu v} f_{v} \\
& +\frac{1}{2} \frac{m^{2}}{n \cdot \partial} n_{\mu} c^{\mu v} \tilde{F}_{v}+\partial_{\mu} \tilde{c}_{2} \partial^{\mu} c_{2} \\
& +m^{2} \tilde{c}_{2} c_{2}+\tilde{f}_{\mu} f_{\mu}-\tilde{F}_{\mu} F^{\mu}+\partial_{\mu} \beta^{\mu} B_{2} \\
& -\frac{1}{2} \frac{m^{2}}{n \cdot \partial} n_{\mu} \beta^{\mu} B_{2}+\partial_{\mu} \phi^{\mu} B_{1}-\partial_{\mu} \tilde{\beta}^{\mu} B \\
& -\frac{1}{2} \frac{m^{2}}{n \cdot \partial} n_{\mu} \phi^{\mu} B_{1}+\frac{1}{2} \frac{m^{2}}{n \cdot \partial} n_{\mu} \tilde{\beta}^{\mu} B .
\end{aligned}
$$

Here, keeping the reducible nature of the theory, we have introduced extra auxiliary and ghost fields. For instance, antisymmetric ghost and antighost fields $c_{\mu \nu}$ and $\bar{c}_{\mu \nu}$ are Grassmannian and the vector field $\phi_{\mu}$, antisymmetric auxiliary fields $B_{\mu \nu}, \bar{B}_{\mu \nu}$ and auxiliary fields $B, B_{1}, B_{2}$ are bosonic. The fields $\beta_{\mu}$ and $\bar{\beta}_{\mu}$ are ghost of ghosts and are bosonic in nature. However, $c_{2}$ and $\bar{c}_{2}$ are ghost of ghost of ghosts with fermionic nature. The rest of the Grassmannian fields, i.e., $c_{1}, \bar{c}_{1}, f_{\mu}$ and $\bar{F}_{\mu}$, are auxiliary fields. It has been shown in Ref. [8] that the ghosts $c_{\mu \nu}$ and $\bar{c}_{\mu \nu}$, ghost of ghosts $\beta_{\mu}$ and $\bar{\beta}_{\mu}$ and ghost of ghost of ghosts $c_{2}$ and $\bar{c}_{2}$, are massive.

To write the absolutely anticommuting BRST and antiBRST invariant BV action for 3-form gauge theory in VSR, we consider an equivalent candidate to the above gaugefixing Lagrangian density as follows:

$$
\begin{aligned}
\mathcal{L}_{g f}^{\tilde{B}}= & -\partial_{\mu} B^{\mu \nu \eta} \tilde{B}_{\nu \eta}+\frac{1}{2} \frac{m^{2}}{n \cdot \partial} n^{\mu} B^{\mu \nu \eta} \tilde{B}_{\nu \eta} \\
& +\frac{1}{2} B_{\mu \nu} \tilde{B}^{\mu \nu}+\partial_{\mu} \tilde{c}_{\nu \eta} \partial^{\mu} c^{\nu \eta}+m^{2} \tilde{c}_{\nu \eta} c^{\nu \eta} \\
& +\partial_{\nu} \tilde{c}_{\eta \mu} \partial^{\mu} c^{\nu \eta}-\partial_{\nu} \tilde{c}_{\eta \mu} \frac{1}{2} \frac{m^{2}}{n \cdot \partial} n^{\mu} c^{\nu \eta} \\
& -\partial^{\mu} c^{\nu \eta} \frac{1}{2} \frac{m^{2}}{n \cdot \partial} n_{\nu} \tilde{c}^{\eta \mu}+n_{\nu} \tilde{c}^{\eta \mu} \frac{1}{4} \frac{m^{2}}{n \cdot \partial} n^{\mu} c^{\nu \eta} \\
& +\partial_{\mu} \tilde{c}_{\mu \nu} \partial^{\mu} c^{\nu \eta}-\partial_{\mu} \tilde{c}_{\mu \nu} \frac{1}{2} \frac{m^{2}}{n \cdot \partial} n^{\mu} c^{\nu \eta} \\
& -\partial^{\mu} c^{\nu \eta} \frac{1}{2} \frac{m^{2}}{n \cdot \partial} n_{\eta} \tilde{c}_{\mu \nu}+n^{\eta} \tilde{c}^{\mu \nu} \frac{1}{4} \frac{m^{2}}{n \cdot \partial} n_{\eta} \tilde{c}_{\mu \nu} \\
& -\partial_{\mu} \tilde{\beta}_{\nu} \partial^{\mu} \beta^{\nu}-m^{2} \tilde{\beta}_{\nu} \beta^{\nu}+\partial_{\nu} \tilde{\beta}_{\mu} \partial^{\mu} \beta^{\nu} \\
& -\partial_{\nu} \tilde{\beta}_{\mu} \frac{1}{2} \frac{m^{2}}{n \cdot \partial} n^{\mu} \beta^{\nu}-\partial^{\mu} \beta^{\nu} \frac{1}{2} \frac{m^{2}}{n \cdot \partial} n_{\nu} \tilde{\beta}_{\mu} \\
& +\frac{1}{4} \frac{m^{2}}{n \cdot \partial} n_{\nu} \tilde{\beta}_{\mu} n^{\mu} \beta^{\nu}-B B_{2}-\frac{1}{2} B_{1}^{2} \\
& +\partial_{\mu} c^{\mu \nu} \tilde{f}_{\nu}-\partial_{\mu} \tilde{c}^{\mu \nu} F_{\nu}+\frac{1}{2} \frac{m^{2}}{n \cdot \partial} n_{\mu} \tilde{c}^{\mu \nu} F_{\nu} \\
& -\frac{1}{2} \frac{m^{2}}{n \cdot \partial} n_{\mu} c^{\mu \nu} \tilde{f}_{\nu}+\partial_{\mu} \tilde{c}_{2} \partial^{\mu} c_{2}+m^{2} \tilde{c}_{2} c_{2} \\
& +\tilde{f}_{\mu} f_{\mu}-\tilde{F}_{\mu} F^{\mu}+\partial_{\mu} \beta^{\mu} B_{2}
\end{aligned}
$$




$$
\begin{aligned}
& -\frac{1}{2} \frac{m^{2}}{n \cdot \partial} n_{\mu} \beta^{\mu} B_{2}+\partial_{\mu} \phi^{\mu} B_{1}-\partial_{\mu} \tilde{\beta}^{\mu} B \\
& -\frac{1}{2} \frac{m^{2}}{n \cdot \partial} n_{\mu} \phi^{\mu} B_{1}+\frac{1}{2} \frac{m^{2}}{n \cdot \partial} n_{\mu} \tilde{\beta}^{\mu} B .
\end{aligned}
$$

These two Lagrangian densities are equivalent as they describe same dynamics of the theory on the following CF type restricted surface:

$$
\begin{aligned}
f_{\mu}+F_{\mu} & =\partial_{\mu} c_{1}-\frac{1}{2} \frac{m^{2}}{n \cdot \partial} n_{\mu} c_{1}, \\
\bar{f}_{\mu}+\bar{F}_{\mu} & =\partial_{\mu} \bar{c}_{1}-\frac{1}{2} \frac{m^{2}}{n \cdot \partial} n_{\mu} \bar{c}_{1}, \\
B_{\mu \nu}+\bar{B}_{\mu \nu} & =\partial_{\mu} \phi_{\nu}-\partial_{\nu} \phi_{\mu}-\frac{1}{2} \frac{m^{2}}{n \cdot \partial} n_{\mu} \phi_{\nu}+\frac{1}{2} \frac{m^{2}}{n \cdot \partial} n_{\nu} \phi_{\mu} .
\end{aligned}
$$

The VSR-modified BRST transformations which leave the effective Lagrangian density, $\mathcal{L}=\mathcal{L}_{0}+\mathcal{L}_{\text {gf }}^{B}$, invariant are given by

$$
\begin{aligned}
s_{b} B_{\mu \nu \eta}= & \left(\partial_{\mu} c_{\nu \eta}+\partial_{\nu} c_{\eta \mu}+\partial_{\eta} c_{\mu \nu}-\frac{1}{2} \frac{m^{2}}{n \cdot \partial} n_{\mu} c_{\nu \eta}\right. \\
& \left.-\frac{1}{2} \frac{m^{2}}{n \cdot \partial} n_{\nu} c_{\eta \mu}-\frac{1}{2} \frac{m^{2}}{n \cdot \partial} n_{\eta} c_{\mu \nu}\right), \\
& s_{b} \tilde{c}_{\mu \nu}=B_{\mu \nu}, \quad s_{b} B_{\mu \nu}=\partial_{\mu} f_{\nu}-\partial_{\nu} f_{\mu} \\
& -\frac{1}{2} \frac{m^{2}}{n \cdot \partial} n_{\mu} f_{\nu}+\frac{1}{2} \frac{m^{2}}{n \cdot \partial} n_{\nu} f_{\mu}, \quad s_{b} \tilde{\beta}_{\mu}=\tilde{F}_{\mu}, \\
& s_{b} \beta_{\mu}=\left(\partial_{\mu}-\frac{1}{2} \frac{m^{2}}{n \cdot \partial} n_{\mu}\right) c_{2}, \\
& s_{b} F_{\mu}=-\left(\partial_{\mu}-\frac{1}{2} \frac{m^{2}}{n \cdot \partial} n_{\mu}\right) B, \\
s_{b} \tilde{f}_{\mu}= & \left(\partial_{\mu}-\frac{1}{2} \frac{m^{2}}{n \cdot \partial} n_{\mu}\right) B_{1}, \\
s_{b} \tilde{c}_{2}= & B_{2}, \quad s_{b} c_{1}=-B, \quad s_{b} \phi_{\mu}=f_{\mu}, \\
s_{b} \tilde{c}_{1}= & B_{1}, \quad s_{b}\left[c_{2}, f_{\mu}, \tilde{F}_{\mu}, B, B_{1}, B_{2}, B_{\mu \nu}\right]=0 . \quad
\end{aligned}
$$

Since the gauge-fixed Lagrangian density $\mathcal{L}_{g f}^{B}$ is BRST exact, so it can be written in terms of BRST variation of a gaugefixing fermion $\Psi$ as

$\mathcal{L}_{g f}^{B}=s_{b} \Psi$,

where the explicit form of $\Psi$ is as follows:

$$
\begin{aligned}
\Psi= & -\frac{1}{2} \tilde{c}_{2} B+\frac{1}{2} B_{2} c_{1}-\frac{1}{2} \tilde{c}_{1} B_{1}-\frac{1}{2}\left(\partial_{\mu} \tilde{\beta}_{\nu}-\partial_{\nu} \tilde{\beta}_{\mu}\right. \\
& \left.-\frac{1}{2} \frac{m^{2}}{n \cdot \partial} n_{\mu} \tilde{\beta}_{\nu}+\frac{1}{2} \frac{m^{2}}{n \cdot \partial} n_{\nu} \tilde{\beta}_{\mu}\right) c^{\mu \nu} \\
& +\frac{1}{2} \tilde{c}_{\mu \nu} \tilde{B}^{\mu \nu}-\partial_{\mu} \tilde{c}_{2} \beta^{\mu}+\frac{1}{2} \frac{m^{2}}{n \cdot \partial}(n \cdot \beta) \tilde{c}_{2}-\tilde{\beta}_{\mu} F^{\mu} \\
& -\phi_{\mu} \tilde{f}^{\mu}-\frac{1}{3} B_{\mu \nu \eta}\left(\partial^{\mu} \tilde{c}^{\nu \eta}+\partial^{\nu} \tilde{c}^{\eta \mu}\right.
\end{aligned}
$$

$$
\begin{aligned}
& +\partial^{\eta} \tilde{c}^{\mu \nu}-\frac{1}{2} \frac{m^{2}}{n \cdot \partial} n^{\mu} \tilde{c}^{\mu \eta}-\frac{1}{2} \frac{m^{2}}{n \cdot \partial} n^{\nu} c^{\eta \mu} \\
& \left.-\frac{1}{2} \frac{m^{2}}{n \cdot \partial} n^{\eta} \tilde{c}^{\mu \nu}\right) .
\end{aligned}
$$

Here, one can see the VSR-modification in the gauge-fixing fermion clearly. The anti-BRST symmetry transformations $\left(s_{a b}\right)$ for the above Lagrangian densities are given by

$$
\begin{aligned}
s_{a b} B_{\mu \nu \eta}= & \left(\partial_{\mu} \tilde{c}_{\nu \eta}+\partial_{\nu} \tilde{c}_{\eta \mu}+\partial_{\eta} \tilde{c}_{\mu \nu}-\frac{1}{2} \frac{m^{2}}{n \cdot \partial} n_{\mu} \tilde{c}_{\nu \eta}\right. \\
& \left.-\frac{1}{2} \frac{m^{2}}{n \cdot \partial} n_{\nu} \tilde{c}_{\eta \mu}-\frac{1}{2} \frac{m^{2}}{n \cdot \partial} n_{\eta} \tilde{c}_{\mu \nu}\right) \\
s_{a b} \tilde{c}_{\mu \nu}= & \partial_{\mu} \tilde{\beta}_{\nu}-\partial_{\nu} \tilde{\beta}_{\mu}-\frac{1}{2} \frac{m^{2}}{n \cdot \partial} n_{\mu} \tilde{\beta}_{\nu} \\
& +\frac{1}{2} \frac{m^{2}}{n \cdot \partial} n_{\nu} \tilde{\beta}_{\mu}, \quad s_{a b} c_{\mu \nu}=\tilde{B}_{\mu \nu}, \\
s_{a b} B_{\mu \nu}= & \partial_{\mu} \tilde{f}_{\nu}-\partial_{\nu} \tilde{f}_{\mu}-\frac{1}{2} \frac{m^{2}}{n \cdot \partial} n_{\mu} \tilde{f}_{\nu} \\
& +\frac{1}{2} \frac{m^{2}}{n \cdot \partial} n_{\nu} \tilde{f}_{\mu}, \quad s_{a b} \beta_{\mu}=F_{\mu}, \\
s_{a b} \tilde{\beta}_{\mu}= & =\partial_{\mu} \tilde{c}_{2}-\frac{1}{2} \frac{m^{2}}{n \cdot \partial} n_{\mu} \tilde{c}_{2}, \\
s_{a b} \tilde{F}_{\mu}= & -\partial_{\mu} B_{2}+\frac{1}{2} \frac{m^{2}}{n \cdot \partial} n_{\mu} B_{2}, \\
s_{a b} f_{\mu}= & -\partial_{\mu} B_{1}+\frac{1}{2} \frac{m^{2}}{n \cdot \partial} n_{\mu} B_{1}, \\
s_{a b} c_{2}= & B, s_{a b} c_{1}=-B_{1}, \quad s_{a b} \phi_{\mu}=\tilde{f}_{\mu}, \\
s_{a b} \tilde{c}_{1}= & -B_{2}, \quad s_{a b}\left[\tilde{c}_{2}, \tilde{f}_{\mu}, F_{\mu}, B, B_{1}, B_{2}, \tilde{B}_{\mu \nu}\right]=0 .
\end{aligned}
$$

Since both the BRST and anti-BRST transformations are absolutely anticommuting in nature, the BRST and antiBRST exact Lagrangian densities can be expressed as follows:

$$
\begin{aligned}
\mathcal{L}_{g f}^{B}= & s_{b} s_{a b}\left[\frac{1}{2} \tilde{c}_{2} c_{2}-\frac{1}{2} \tilde{c}_{1} c_{1}-\frac{1}{2} \tilde{c}_{\mu \nu} c^{\mu \nu}\right. \\
& \left.-\tilde{\beta}_{\mu} \beta^{\mu}-\frac{1}{2} \phi_{\mu} \phi^{\mu}-\frac{1}{6} B_{\mu \nu \eta} B^{\mu \nu \eta}\right] .
\end{aligned}
$$

Now, we will study the construction of VSR-modified extended BRST transformation for Abelian 3-form gauge theory in next subsection.

\subsection{VSR-modified extended BRST invariant BV action}

Following the previous sections, we generalize the VSRmodified extended BRST construction for the case of Abelian 3 -form gauge theory in VSR. In this regard, we shift all the fields from their original values as follows: 


$$
\begin{aligned}
& B_{\mu \nu \eta} \rightarrow B_{\mu \nu \eta}-\bar{B}_{\mu \nu \eta}, c_{\mu \nu} \rightarrow c_{\mu \nu}-\bar{c}_{\mu \nu}, \\
& \tilde{c}_{\mu \nu} \rightarrow \tilde{c}_{\mu \nu}-\overline{\tilde{c}}_{\mu \nu}, \quad B_{\mu \nu} \rightarrow B_{\mu \nu}-\bar{B}_{\mu \nu}, \\
& \tilde{B}_{\mu \nu} \rightarrow \tilde{B}_{\mu \nu}-\overline{\tilde{B}}_{\mu \nu}, \quad \beta_{\mu} \rightarrow \beta_{\mu}-\bar{\beta}_{\mu}, \\
& \tilde{\beta}_{\mu} \rightarrow \tilde{\beta}_{\mu}-\overline{\tilde{\beta}}_{\mu}, \quad F_{\mu} \rightarrow F_{\mu}-\bar{F}_{\mu}, \\
& \tilde{F}_{\mu} \rightarrow \tilde{F}_{\mu}-\overline{\tilde{F}}_{\mu}, \quad f_{\mu} \rightarrow f_{\mu}-\bar{f}_{\mu}, \\
& \tilde{f}_{\mu} \rightarrow \tilde{f}_{\mu}-\overline{\tilde{f}}_{\mu}, \quad c_{2} \rightarrow c_{2}-\bar{c}_{2}, \\
& \tilde{c}_{2} \rightarrow \tilde{c}_{2}-\overline{\tilde{c}}_{2}, \quad c_{1} \rightarrow c_{1}-\bar{c}_{1}, \tilde{c}_{1} \rightarrow \tilde{c}_{1}-\overline{\tilde{c}}_{1}, \\
& \phi_{\mu} \rightarrow \phi_{\mu}-\bar{\phi}_{\mu}, \\
& B \rightarrow B-\bar{B}, \quad B_{1} \rightarrow B_{1}-\bar{B}_{1}, \quad B_{2} \rightarrow B_{2}-\bar{B}_{2} .
\end{aligned}
$$

Following the above shifts in fields, the effective Lagrangian density of the theory is modified by

$$
\begin{aligned}
\overline{\mathcal{L}}= & \mathcal{L}\left(B_{\mu \nu \eta}-\bar{B}_{\mu \nu \eta}, c_{\mu \nu}-\bar{c}_{\mu \nu}, \tilde{c}_{\mu \nu}-\overline{\tilde{c}}_{\mu \nu}, B_{\mu \nu}-\bar{B}_{\mu \nu},\right. \\
& \tilde{B}_{\mu \nu}-\overline{\tilde{B}}_{\mu \nu}, \beta_{\mu}-\bar{\beta}_{\mu}, \tilde{\beta}_{\mu}-\overline{\tilde{\beta}}_{\mu}, \\
& F_{\mu}-\bar{F}_{\mu}, \tilde{F}_{\mu}-\overline{\tilde{F}}_{\mu}, f_{\mu}-\bar{f}_{\mu}, \tilde{f}_{\mu}-\overline{\tilde{f}}_{\mu}, c_{2}-\bar{c}_{2}, \tilde{c}_{2} \\
& -\overline{\tilde{c}}_{2}, c_{1}-\bar{c}_{1}, \tilde{c}_{1}-\overline{\tilde{c}}_{1}, \phi_{\mu}-\bar{\phi}_{\mu}, \\
& \left.B-\bar{B}, B_{1}-\bar{B}_{1}, B_{2}-\bar{B}_{2}\right) .
\end{aligned}
$$

This Lagrangian density is invariant under the BRST transformation (4.7) with respect to the shifted fields. In addition, it is also invariant under the extended BRST transformations of the fields given as

$s_{b} \Phi(x)=\alpha(x), \quad s_{b} \Phi(x)=\alpha(x)-\beta(x)$,

where $\Phi(x)$ and $\bar{\Phi}(x)$ represent the collective original and shifted fields, respectively. Here $\beta(x)$ refers the original BRST transformation of with respect to the shifted fields, whereas $\alpha(x)$ corresponds the ghost fields associated with shift symmetry collectively. The explicit form of the extended BRST transformation (4.14) is given explicitly in Eq. (B2) of Appendix B. Now, in order to make the theory unchanged, we need to remove the contribution of these ghosts from the physical states. To do so, we further introduce the following antifields (antighosts): $B_{\mu \nu \eta}^{\star}, c_{\mu \nu}^{\star}, \tilde{c}_{\mu \nu}^{\star}, B_{\mu \nu}^{\star}, \tilde{B}_{\mu \nu}^{\star}, \eta_{\mu}^{\star}$, $\tilde{\beta}_{\mu}^{\star}, F_{\mu}^{\star}, \tilde{F}_{\mu}^{\star}, f_{\mu}^{\star}, \tilde{f}_{\mu}^{\star}, c_{2}^{\star}, \tilde{c}_{2}^{\star}, c_{1}^{\star}, \tilde{c}_{1}^{\star}, \phi_{\mu}^{\star}, B^{\star}, B_{1}^{\star}, B_{2}^{\star}$ to the theory. The BRST variation of these antifields are given in (B3). Now, to remove the redundancies, we fix the gauge for shift symmetry, which is achieved by adding the following gauge-fixing term to the VSR quantum action:

$$
\begin{aligned}
\overline{\mathcal{L}}_{g f}^{B}= & l_{\mu \nu \eta} \bar{B}^{\mu \nu \eta}-B_{\mu \nu \eta}^{\star}\left(L^{\mu \nu \eta}-\partial^{\mu} c^{\nu \eta}+\partial^{\mu} \bar{c}^{\nu \eta}\right. \\
& -\partial^{\nu} c^{\eta \mu}+\partial^{\nu} \bar{c}^{\eta \mu}-\partial^{\eta} c^{\mu \nu}+\partial^{\eta} \bar{c}^{\mu \nu} \\
& +\frac{1}{2} \frac{m^{2}}{n \cdot \partial} n^{\mu} c^{\nu \eta}-\frac{1}{2} \frac{m^{2}}{n \cdot \partial} n^{\mu} \bar{c}^{\nu \eta}+\frac{1}{2} \frac{m^{2}}{n \cdot \partial} n^{\nu} c^{\eta \mu} \\
& -\frac{1}{2} \frac{m^{2}}{n \cdot \partial} n^{\nu} \bar{c}^{\eta \mu}+\frac{1}{2} \frac{m^{2}}{n \cdot \partial} n^{\eta} c^{\mu \nu} \\
- & \left.\frac{1}{2} \frac{m^{2}}{n \cdot \partial} n^{\eta} \bar{c}^{\mu \nu}\right)+\tilde{c}_{\mu \nu}^{\star}\left(M^{\mu \nu}-\partial^{\mu} \beta^{\nu}+\partial^{\mu} \bar{\beta}^{\nu}\right.
\end{aligned}
$$

$$
\begin{aligned}
& +\partial^{v} \beta^{\mu}-\partial^{v} \bar{\beta}^{\mu}+\frac{1}{2} \frac{m^{2}}{n \cdot \partial} n^{\mu} \beta^{\nu} \\
& \left.-\frac{1}{2} \frac{m^{2}}{n \cdot \partial} n^{\mu} \bar{\beta}^{\nu}-\frac{1}{2} \frac{m^{2}}{n \cdot \partial} n^{\nu} \beta^{\mu}+\frac{1}{2} \frac{m^{2}}{n \cdot \partial} n^{\nu} \bar{\beta}^{\mu}\right) \\
& +m_{\mu \nu} \overline{\tilde{c}}^{\mu \nu}+c_{\mu \nu}^{\star}\left(\tilde{M}^{\mu \nu}-B^{\mu \nu}+\bar{B}^{\mu \nu}\right) \\
& +n_{\mu \nu} \bar{B}^{\mu \nu}-B_{\mu \nu}^{\star} N^{\mu \nu}+\bar{n}_{\mu \nu} \overline{\tilde{B}}^{\mu \nu} \\
& -\tilde{B}_{\mu \nu}^{\star}\left(\tilde{N}^{\mu \nu}-\partial^{\mu} F^{\nu}+\partial^{\mu} \bar{F}^{\nu}+\partial^{\nu} F^{\mu}\right. \\
& -\partial^{v} \bar{F}^{\mu}+\frac{1}{2} \frac{m^{2}}{n \cdot \partial} n^{\mu} F^{v}-\frac{1}{2} \frac{m^{2}}{n \cdot \partial} n^{\mu} \bar{F}^{v} \\
& \left.-\frac{1}{2} \frac{m^{2}}{n \cdot \partial} n^{\nu} F^{\mu}+\frac{1}{2} \frac{m^{2}}{n \cdot \partial} n^{\nu} \bar{F}^{\mu}\right)+o_{\mu} \bar{\beta}^{\mu} \\
& -\beta_{\mu}^{\star}\left(O^{\mu}-\partial^{\mu} c_{2}+\partial^{\mu} \bar{c}_{2}+\frac{1}{2} \frac{m^{2}}{n \cdot \partial} n^{\mu} c_{2}\right. \\
& \left.-\frac{1}{2} \frac{m^{2}}{n \cdot \partial} n^{\mu} \bar{c}_{2}\right)+\bar{o}_{\mu} \overline{\tilde{\beta}}^{\mu}-\tilde{\beta}_{\mu}^{\star}\left(\tilde{O}^{\mu}-\tilde{F}^{\mu}\right. \\
& \left.+\overline{\tilde{F}}^{\mu}\right)+\bar{p}_{\mu} \bar{F}^{\mu}+\tilde{F}_{\mu}^{\star}\left(P^{\mu}+\partial^{\mu} B-\partial^{\mu} \bar{B}\right. \\
& \left.-\frac{1}{2} \frac{m^{2}}{n \cdot \partial} n^{\mu} B+\frac{1}{2} \frac{m^{2}}{n \cdot \partial} n^{\mu} \bar{B}\right)+p_{\mu} \overline{\tilde{F}}^{\mu} \\
& +F_{\mu}^{\star} \tilde{P}^{\mu}+\bar{q}_{\mu} \bar{f}^{\mu}+\tilde{f}_{\mu}^{\star} Q^{\mu}+q_{\mu}{\tilde{f^{\mu}}}^{-\mu} f_{\mu}^{\star}\left(\tilde{Q}^{\mu}\right. \\
& -\partial^{\mu} B_{1}+\partial^{\mu} \bar{B}_{1}+\frac{1}{2} \frac{m^{2}}{n \cdot \partial} n^{\mu} B_{1} \\
& \left.-\frac{1}{2} \frac{m^{2}}{n \cdot \partial} n^{\mu} \bar{B}_{1}\right)+\bar{r} \bar{c}_{2}+\tilde{c}_{2}^{\star} \mathfrak{R}+r \overline{\tilde{c}}_{2} \\
& +c_{2}^{\star}\left(\tilde{\Re}-B_{2}+\bar{B}_{2}\right)+\bar{s} \bar{c}_{1}+s \overline{\tilde{c}}_{1}+v \bar{B}_{1} \\
& +\tilde{c}_{1}^{\star}(\mathfrak{S}+B-\bar{B})+c_{1}^{\star}\left(\tilde{\mathfrak{S}}-B_{1}+\bar{B}_{1}\right) \\
& +t_{\mu} \bar{\phi}^{\mu}-\phi_{\mu}^{\star}\left(T^{\mu}-f^{\mu}+\bar{f}^{\mu}\right)+u \bar{B} \\
& -B^{\star} \mathbb{U}+w \bar{B}_{2}-B_{2}^{\star} \mathfrak{W}-B_{1}^{\star} \mathfrak{V} .
\end{aligned}
$$

where the fields $L_{\mu \nu \eta}, M_{\mu \nu}, \tilde{M}_{\mu \nu}, N_{\mu \nu}, \tilde{N}_{\mu \nu}, O_{\mu}, \tilde{O}_{\mu}, P_{\mu}$, $\tilde{P}_{\mu}, Q_{\mu}, \tilde{Q}_{\mu}, \mathfrak{R}, \tilde{\mathfrak{R}}, \mathfrak{S}, \tilde{\mathfrak{S}}, T_{\mu}, \mathbb{U}, \mathfrak{V}$ and $\mathfrak{W}$ are the ghost fields associated with the shift symmetries for fields $B_{\mu \nu \eta}$, $c_{\mu \nu}, \tilde{c}_{\mu \nu}, B_{\mu \nu}, \tilde{B}_{\mu \nu}, \beta_{\mu}, \tilde{\beta}_{\mu}, F_{\mu}, \tilde{F}_{\mu}, f_{\mu}, \tilde{f}_{\mu}, c_{2}, \tilde{c}_{2}, c_{1}, \tilde{c}_{1}$, $\phi_{\mu}, B, B_{1}, B_{2}$ respectively and the fields $l_{\mu \nu \eta}, m_{\mu \nu}, \bar{m}_{\mu \nu}$, $n_{\mu \nu}, \bar{n}_{\mu \nu}, o_{\mu}, \bar{o}_{\mu}, p_{\mu}, \bar{p}_{\mu}, q_{\mu}, \bar{q}_{\mu}, r, \bar{r}, s, \bar{s}, t_{\mu}, u, v, w$ are the Nakanishi-Lautrup type auxiliary fields corresponding to the antighost fields $B_{\mu \nu \eta}^{\star}, c_{\mu \nu}^{\star}, \tilde{c}_{\mu \nu}^{\star}, B_{\mu \nu}^{\star}, \tilde{B}_{\mu \nu}^{\star}, \eta_{\mu}^{\star}, \tilde{\beta}_{\mu}^{\star}, F_{\mu}^{\star}$, $\tilde{F}_{\mu}^{\star}, f_{\mu}^{\star}, \tilde{f}_{\mu}^{\star}, c_{2}^{\star}, \tilde{c}_{2}^{\star}, c_{1}^{\star}, \tilde{c}_{1}^{\star}, \phi_{\mu}^{\star}, B^{\star}, B_{1}^{\star}, B_{2}^{\star}$, respectively.

The gauge-fixed Lagrangian density corresponding to shift symmetry, $\overline{\mathcal{L}}_{g f}^{B}$, is invariant under the extended BRST symmetry transformations given in Eqs. (B2) and (B3) of Appendix B. If we perform the equations of motion for the auxiliary fields, then all bar fields disappear and we left with the following gauge-fixed Lagrangian density:

$\overline{\mathcal{L}}_{g f}^{B}=-B_{\mu \nu \eta}^{\star}\left(L^{\mu \nu \eta}-\partial^{\mu} c^{\nu \eta}-\partial^{\nu} c^{\eta \mu}-\partial^{\eta} c^{\mu \nu}\right.$ 


$$
\begin{aligned}
& +\frac{1}{2} \frac{m^{2}}{n \cdot \partial} n^{\mu} c^{\nu \eta}+\frac{1}{2} \frac{m^{2}}{n \cdot \partial} n^{\nu} c^{\eta \mu} \\
& \left.+\frac{1}{2} \frac{m^{2}}{n \cdot \partial} n^{\eta} c^{\mu \nu}\right)+\tilde{c}_{\mu \nu}^{\star}\left(M^{\mu \nu}-\tilde{\partial}^{\mu} \beta^{v}+\tilde{\partial}^{v} \beta^{\mu}\right. \\
& \left.+\frac{1}{2} \frac{m^{2}}{n \cdot \partial} n^{\mu} \beta^{v}-\frac{1}{2} \frac{m^{2}}{n \cdot \partial} n^{v} \beta^{\mu}\right) \\
& +c_{\mu \nu}^{\star}\left(\tilde{M}^{\mu \nu}-B^{\mu \nu}\right)-B_{\mu \nu}^{\star} N^{\mu \nu}+\tilde{B}_{\mu \nu}^{\star}\left(\tilde{N}^{\mu \nu}\right. \\
& -\partial^{\mu} f^{v}+\partial^{v} f^{\mu}+\frac{1}{2} \frac{m^{2}}{n \cdot \partial} n^{\mu} f^{v} \\
& \left.-\frac{1}{2} \frac{m^{2}}{n \cdot \partial} n^{v} f^{\mu}\right)-\beta_{\mu}^{\star}\left(O^{\mu}-\partial^{\mu} c_{2}\right. \\
& \left.+\frac{1}{2} \frac{m^{2}}{n \cdot \partial} n^{\mu} c_{2}\right)+\tilde{\beta}_{\mu}^{\star}\left(\tilde{O}^{\mu}-\tilde{F}^{\mu}\right) \\
& +\tilde{F}_{\mu}^{\star}\left(P^{\mu}+\partial^{\mu} B-\frac{1}{2} \frac{m^{2}}{n \cdot \partial} n^{\mu} B\right) \\
& +F_{\mu}^{\star} \tilde{P}^{\mu}+\tilde{f}_{\mu}^{\star} Q^{\mu}+f_{\mu}^{\star}\left(\tilde{Q}^{\mu}-\partial^{\mu} B_{1}\right. \\
& \left.+\frac{1}{2} \frac{m^{2}}{n \cdot \partial} n^{\mu} B_{1}\right)+\tilde{c}_{2}^{\star} \mathfrak{R}+c_{2}^{\star}\left(\tilde{\Re}-B_{2}\right) \\
& +\tilde{c}_{1}^{\star}(\mathfrak{S}+B)+c_{1}^{\star}\left(\tilde{\mathfrak{S}}-B_{1}\right) \\
& -\phi_{\mu}^{\star}\left(T^{\mu}-f^{\mu}\right)-B^{\star} \mathbb{U}-B_{1}^{\star} \mathfrak{V}-B_{2}^{\star} \mathfrak{W} \text {. }
\end{aligned}
$$

Furthermore, if the general gauge-fixing fermion of the Abelian 3-form gauge theory in VSR, $\Psi$, depends on the original fields only, then we can write a general gauge-fixed Lagrangian density for this theory with the original BRST symmetry in terms of $\Psi$ as

$\mathcal{L}_{g f}^{B}=s_{b} \Psi[\Phi]=\sum_{\Phi}\left(s_{b} \Phi\right) \frac{\delta \Psi}{\delta \Phi}$,

where $\Phi$ is the generic notation for all fields in the theory. Keeping the nature of the fields (i.e. fermionic and bosonic) in mind the above gauge-fixed Lagrangian density in can be expressed as

$$
\begin{aligned}
\mathcal{L}_{g f}^{B}= & -\frac{\delta \Psi}{\delta B_{\mu \nu \eta}} L_{\mu \nu \eta}+\frac{\delta \Psi}{\delta c_{\mu \nu}} M_{\mu \nu}+\frac{\delta \Psi}{\delta \tilde{c}_{\mu \nu}} \tilde{M}_{\mu \nu} \\
& -\frac{\delta \Psi}{\delta B_{\mu \nu}} N_{\mu \nu}-\frac{\delta \Psi}{\delta \tilde{B}_{\mu \nu}} \tilde{N}_{\mu \nu} \\
& -\frac{\delta \Psi}{\delta \beta_{\mu}} O_{\mu}-\frac{\delta \Psi}{\delta \tilde{\beta}_{\mu}} \tilde{O}_{\mu}+\frac{\delta \Psi}{\delta F_{\mu}} P_{\mu}+\frac{\delta \Psi}{\delta \tilde{F}_{\mu}} \tilde{P}_{\mu} \\
& +\frac{\delta \Psi}{\delta f_{\mu}} Q_{\mu}+\frac{\delta \Psi}{\delta \tilde{f}_{\mu}} \tilde{Q}_{\mu} \\
& +\frac{\delta \Psi}{\delta c_{2}} \mathfrak{R}+\frac{\delta \Psi}{\delta \tilde{c}_{2}} \tilde{\mathfrak{R}}+\frac{\delta \Psi}{\delta c_{1}} \mathfrak{S}+\frac{\delta \Psi}{\delta \tilde{c}_{1}} \tilde{\mathfrak{S}} \\
& -\frac{\delta \Psi}{\delta \phi_{\mu}} T_{\mu}-\frac{\delta \Psi}{\delta B} \mathbb{U}-\frac{\delta \Psi}{\delta B_{1}} \mathfrak{V}-\frac{\delta \Psi}{\delta B_{2}} \mathfrak{W} .
\end{aligned}
$$

Now, we are able to define the BV action for Abelian 3-form gauge theory in VSR by combining Eqs. (4.4), (4.16) and (4.18) as follows:

$\mathcal{L}_{\text {eff }}=\mathcal{L}_{0}\left(B_{\mu \nu \rho}-\bar{B}_{\mu \nu \rho}\right)+\mathcal{L}_{g f}^{B}+\overline{\mathcal{L}}_{g f}^{B}$.

The explicit expression of $\mathcal{L}_{\text {eff }}$ is given in (B1) (see Appendix B).

To obtain the identifications on the antifields of 3-form theory in VSR, we integrate the ghosts associated with the shift symmetry and utilize the gauge-fixing fermion (4.9). We thus obtain the explicit values of the antifields as follows:

$$
\begin{aligned}
& B^{\mu \nu \eta \star}=\frac{1}{3}\left(\partial^{\mu} \tilde{c}^{\nu \eta}+\partial^{\nu} \tilde{c}^{\eta \mu}+\partial^{\eta} \tilde{c}^{\mu \nu}-\frac{1}{2} \frac{m^{2}}{n \cdot \partial} n^{\mu} \tilde{c}^{\nu \eta}\right. \\
& \left.-\frac{1}{2} \frac{m^{2}}{n \cdot \partial} n^{\nu} \tilde{c}^{\eta \mu}-\frac{1}{2} \frac{m^{2}}{n \cdot \partial} n^{\eta} \tilde{c}^{\mu \nu}\right) \text {, } \\
& c^{\mu \nu \star}=-\frac{1}{2} B^{\mu \nu}, \quad B^{\mu \nu \star}=-\frac{1}{2} \tilde{c}^{\mu \nu}, \\
& \tilde{\beta}^{\mu \star}=F^{\mu}+\partial_{\nu} c^{\mu \nu}-\frac{1}{2} \frac{m^{2}}{n \cdot \partial} n_{\nu} c^{\mu \nu}, \\
& \tilde{c}^{\mu \nu \star}=\frac{1}{2}\left(\partial^{\mu} \tilde{\beta}^{\nu}-\partial^{\nu} \tilde{\beta}^{\mu}-\frac{1}{2} \frac{m^{2}}{n \cdot \partial} n^{\mu} \tilde{\beta}^{\nu}+\frac{1}{2} \frac{m^{2}}{n \cdot \partial} n^{\nu} \tilde{\beta}^{\mu}\right) \\
& -\partial_{\eta} B^{\mu \nu \eta}+\frac{1}{2} \frac{m^{2}}{n \cdot \partial} n_{\eta} B^{\mu \nu \eta} \\
& \beta^{\mu \star}=-\frac{1}{2} \partial^{\mu} \tilde{c}_{2}+\frac{1}{4} \frac{m^{2}}{n \cdot \partial} n^{\mu} \tilde{c}_{2}, c_{2}^{\star}=\frac{1}{2} B \\
& -\left(\partial_{\mu}-\frac{1}{2} \frac{m^{2}}{n \cdot \partial} n_{\mu}\right) \beta^{\mu}, \tilde{c}_{1}^{\star}=-\frac{1}{2} B_{2}, \\
& \tilde{F}^{\mu \star}=\tilde{\beta}^{\mu}, f^{\mu \star}=\phi^{\mu}, \quad c_{1}^{\star}=\frac{1}{2} B_{1}, \phi^{\mu \star}=\tilde{f}^{\mu}, \\
& B^{\star}=\frac{1}{2} \tilde{c}_{2}, B_{1}^{\star}=\frac{1}{2} \tilde{c}_{1}, B_{2}^{\star}=-\frac{1}{2} c_{1}, \\
& {\left[\tilde{B}^{\mu \nu \star}, F^{\mu \star}, f^{\mu \star}, \tilde{c}_{2}^{\star}\right]=0 .}
\end{aligned}
$$

Now, we are able to express gauge-fixed Lagrangian density corresponding to extended BRST transformations in terms of generalized gauge-fixing fermion as follows:

$$
\begin{aligned}
\mathcal{L}_{g f}^{B}+\overline{\mathcal{L}}_{g f}^{B}= & s_{b}\left(B_{\mu \nu \eta}^{\star} \bar{B}^{\mu \nu \eta}+c_{\mu \nu}^{\star} \overline{\tilde{c}}^{\mu \nu}+\tilde{c}_{\mu \nu}^{\star} \bar{c}^{\mu \nu}+B_{\mu \nu}^{\star} \bar{B}^{\mu \nu}\right. \\
& +\beta_{\mu}^{\star} \bar{\beta}^{\mu}+\tilde{\beta}_{\mu}^{\star} \overline{\tilde{\beta}}^{\mu}+F_{\mu}^{\star} \overline{\tilde{F}}^{\mu}+\tilde{F}_{\mu}^{\star} \bar{F}^{\mu} \\
& +f_{\mu}^{\star} \overline{\tilde{f}}^{\mu}+\tilde{f}_{\mu}^{\star} \bar{f}^{\mu}+c_{2}^{\star} \overline{\tilde{c}}_{2}+\tilde{c}_{2}^{\star} \bar{c}_{2}+c_{1}^{\star} \overline{\tilde{c}}_{1}+\tilde{c}_{1}^{\star} \bar{c}_{1} \\
& \left.+\phi_{\mu}^{\star} \bar{\phi}^{\mu}+B^{\star} \bar{B}+B_{1}^{\star} \bar{B}_{1}+B_{2}^{\star} \bar{B}_{2}\right), \\
=: & s_{b}\left(\Phi^{\star} \bar{\Phi}\right),
\end{aligned}
$$

where the collective fields $\Phi$ and $\bar{\Phi}$ denote the original fields and corresponding shifted fields, respectively. Here the ghost number of the expression $\Phi^{\star} \bar{\Phi}=-1$, as we expect. Here we see a difference in the expression of generalized gauge-fixing fermion to that of the ordinary gauge-fixing fermion 


$$
\begin{aligned}
\Psi= & -\left[B_{\mu \nu \eta} B^{\mu \nu \eta \star}+\tilde{c}_{\mu \nu} c^{\mu \nu \star}+\tilde{\beta}_{\mu} \tilde{\beta}^{\mu \star}\right. \\
& \left.+\phi_{\mu} \phi^{\mu \star}+\tilde{c}_{2} c_{2}^{\star}+\tilde{c}_{1} c_{1}^{\star}+B_{2} B_{2}^{\star}\right] .
\end{aligned}
$$

Plugging the values of the antifields (4.20), we can recover the original Lagrangian density of 3-form gauge theory in VSR.

\subsection{VSR-modified extended BRST invariant BV action in superspace}

Furthermore, while the BRST and the anti-BRST symmetries of 3-form theories can be given a geometrical meaning and have led to a superspace formulation of such theories [47], a superspace description of the VSR-modified BV action does not exist so far. Here, we develop a superspace formalism of the VSR-modified 3-form theory having extended BRST invariance only. To study the theory with extended BRST invariance only, we need one extra fermionic parameter $\theta$ together with $x_{\mu}$. The components of superfields, $\mathfrak{T}$, in terms of generic fields $\Phi$ is given by

$\mathfrak{T}(x, \theta)=\Phi(x)+\theta\left(s_{b} \Phi\right)$.

With the help of extended BRST transformation (B2), the explicit expressions for the superfield are listed in Eq. (B4) of Appendix.

The gauge-fixed Lagrangian density corresponding to shift symmetry mentioned in (4.15) in the superspace is simplified as

$$
\begin{aligned}
\overline{\mathcal{L}}_{g f}^{B}= & \frac{\delta}{\delta \theta}\left[\overline{\mathcal{B}}_{\mu \nu \eta}^{\star} \overline{\mathcal{B}}^{\mu \nu \eta}+\overline{\tilde{\mathcal{C}}}_{\mu \nu}^{\star} \overline{\mathcal{C}}^{\mu \nu}+\overline{\tilde{\mathcal{C}}}_{\mu \nu} \overline{\mathcal{C}}^{\mu \nu \star}\right. \\
& +\overline{\tilde{\mathcal{B}}}_{\mu \nu}^{\star} \overline{\tilde{\mathcal{B}}}^{\mu \nu}+\overline{\mathcal{B}}_{\mu}^{\star} \overline{\mathcal{B}}^{\mu}+\overline{\tilde{\mathcal{F}}}_{\mu}^{\star} \overline{\mathcal{F}}^{\mu}+\overline{\tilde{\mathfrak{F}}}_{\mu}^{\star} \overline{\mathfrak{F}}^{\mu} \\
& +\overline{\tilde{\mathcal{F}}}_{\mu} \overline{\mathcal{F}}^{\mu \star}+\overline{\tilde{\mathfrak{F}}}_{\mu} \overline{\mathfrak{F}}^{\mu \star}+\overline{\tilde{\mathcal{C}}}_{1}^{\star} \overline{\mathcal{C}}_{1}+\overline{\tilde{\mathcal{C}}}_{1} \overline{\mathcal{C}}_{1}^{\star}+\overline{\tilde{\mathcal{C}}}_{2}^{\star} \overline{\mathcal{C}}_{2} \\
& \left.+\overline{\tilde{\mathcal{C}}}_{2} \overline{\mathcal{C}}_{2}^{\star}+\overline{\mathcal{B}}^{\star} \overline{\mathcal{B}}+\overline{\mathcal{B}}_{1}^{\star} \overline{\mathcal{B}}_{1}+\overline{\mathcal{B}}_{2}^{\star} \overline{\mathcal{B}}_{2}\right], \\
= & \frac{\delta}{\delta \theta}\left[\overline{\mathfrak{T}}^{\star} \overline{\mathfrak{T}}\right] .
\end{aligned}
$$

Being the $\theta$ component of a superfields, $\overline{\mathcal{L}}_{g f}^{B}$ is invariant under the extended BRST transformation. In the extended BRST invariant superspace, the gauge-fixed fermion of the original VSR-modified 3-form gauge theory in component form translates as

$\Gamma(x, \theta)=\Psi(x)+\theta s_{b} \Psi$.

If we assume a general gauge-fixed fermion $\Psi$ depending on all the original fields, then $\Gamma(x, \theta)$ reads

$$
\begin{aligned}
\Gamma(x, \theta)= & \Psi(x)+\theta\left[-\frac{\delta \Psi}{\delta B_{\mu \nu \eta}} L_{\mu \nu \eta}+\frac{\delta \Psi}{\delta c_{\mu \nu}} M_{\mu \nu}\right. \\
& +\frac{\delta \Psi}{\delta \tilde{c}_{\mu \nu}} \tilde{M}_{\mu \nu}-\frac{\delta \Psi}{\delta B_{\mu \nu}} N_{\mu \nu}-\frac{\delta \Psi}{\delta \tilde{B}_{\mu \nu}} \tilde{N}_{\mu \nu} \\
& -\frac{\delta \Psi}{\delta \beta_{\mu}} O_{\mu}-\frac{\delta \Psi}{\delta \tilde{\beta}_{\mu}} \tilde{O}_{\mu}+\frac{\delta \Psi}{\delta F_{\mu}} P_{\mu}+\frac{\delta \Psi}{\delta \tilde{F}_{\mu}} \tilde{P}_{\mu} \\
& +\frac{\delta \Psi}{\delta f_{\mu}} Q_{\mu}+\frac{\delta \Psi}{\delta \tilde{f}_{\mu}} \tilde{Q}_{\mu}+\frac{\delta \Psi}{\delta c_{2}} \mathfrak{R} \\
& +\frac{\delta \Psi}{\delta \tilde{c}_{2}} \tilde{\mathfrak{R}}+\frac{\delta \Psi}{\delta c_{1}} \mathfrak{S}+\frac{\delta \Psi}{\delta \tilde{c}_{1}} \tilde{\mathfrak{S}}-\frac{\delta \Psi}{\delta \phi_{\mu}} T_{\mu} \\
& \left.-\frac{\delta \Psi}{\delta B} \mathbb{U}-\frac{\delta \Psi}{\delta B_{1}} \mathfrak{V}-\frac{\delta \Psi}{\delta B_{2}} \mathfrak{W}\right] .
\end{aligned}
$$

Therefore, the VSR-modified gauge-fixing Lagrangian density (4.4) in the superspace formalism takes a very compact form:

$\mathcal{L}_{g f}^{B}=\frac{\delta \Gamma(x, \theta)}{\delta \theta}$.

Here we see again that $\theta$ being the component of a superfield, this Lagrangian density is manifestly invariant under the extended BRST transformation. Now, we are able to write the extended BRST invariant BV action in the superspace as follows:

$\mathcal{L}_{\text {eff }}=\mathcal{L}_{0}\left(B_{\mu \nu \rho}-\bar{B}_{\mu \nu \rho}\right)+\frac{\delta \Gamma(x, \theta)}{\delta \theta}+\frac{\delta}{\delta \theta}\left[\overline{\mathfrak{T}}^{\star} \overline{\mathfrak{T}}\right]$.

Here we observe that eliminating auxiliary fields and ghost fields of the shift symmetry through using the equations of motion, this effective Lagrangian density reduces to the original BRST invariant Lagrangian density of 3-form gauge theory in VSR also.

\subsection{VSR-modified extended anti-BRST invariant BV action}

In this subsection, we discuss the extended anti-BRST symmetry for Abelian 3-form gauge theory in VSR and their superspace description.

As the gauge-fixing Lagrangian density is anti-BRST exact, so one can write this in terms of corresponding gaugefixing fermion $\bar{\Psi}$ as

$\mathcal{L}_{g f}^{B}=s_{a b} \bar{\Psi}$,

where $\bar{\Psi}$ fhas the following expression:

$$
\begin{aligned}
\bar{\Psi}= & -\frac{1}{2} B_{2} c_{2}+\frac{1}{2} B_{1} c_{1}+\frac{1}{2} \tilde{c}_{1} B+\frac{1}{2} B_{\mu \nu} c^{\mu \nu}-\frac{1}{2} \tilde{c}_{\mu \nu} \\
& \times\left(\partial^{\mu} \beta^{\nu}-\partial^{\nu} \beta^{\mu}-\frac{1}{2} \frac{m^{2}}{n \cdot \partial} n^{\mu} \beta^{\nu}+\frac{1}{2} \frac{m^{2}}{n \cdot \partial} n^{\nu} \beta^{\mu}\right)
\end{aligned}
$$




$$
\begin{aligned}
& +\tilde{F}_{\mu} \beta^{\mu}+\beta_{\mu} \partial^{\mu} c_{2}-\beta_{\mu} \frac{1}{2} \frac{m^{2}}{n \cdot \partial} n^{\mu} c_{2} \\
& +\frac{1}{2} \phi_{\mu} f^{\mu}+\frac{1}{3} B_{\mu \nu \eta}\left(\partial^{\mu} c^{\nu \eta}+\partial^{\nu} c^{\eta \mu}+\partial^{\eta} c^{\mu \nu}\right. \\
& \left.-\frac{1}{2} \frac{m^{2}}{n \cdot \partial} n^{\mu} c^{\nu \eta}-\frac{1}{2} \frac{m^{2}}{n \cdot \partial} n^{\nu} c^{\eta \mu}-\frac{1}{2} \frac{m^{2}}{n \cdot \partial} n^{\eta} c^{\mu \nu}\right) .
\end{aligned}
$$

Keeping the structure of (B2) (given in Appendix B) in mind, we demand that anti-BRST transformation of shifted fields $(\Phi-\bar{\Phi})$ coincides with the anti-BRST transformations of the original fields $(\Phi)$ if all the bar fields vanish. This requirement leads to the following form of the (VSR-modified) extended anti-BRST transformations:

$$
\begin{aligned}
& s_{a b} \bar{B}_{\mu \nu \eta}=B_{\mu \nu \eta}^{\star}, \\
& s_{a b} B_{\mu \nu \eta}=B_{\mu \nu \eta}^{\star}+\partial_{\mu} \tilde{c}_{\nu \eta}-\partial_{\mu} \overline{\tilde{c}}_{\nu \eta}+\partial_{\nu} \tilde{c}_{\eta \mu}-\partial_{\nu} \overline{\tilde{c}}_{\eta \mu} \\
& +\partial_{\eta} \tilde{c}_{\mu \nu}-\partial_{\eta} \overline{\tilde{c}}_{\mu \nu}-\frac{1}{2} \frac{m^{2}}{n \cdot \partial} n_{\mu} \tilde{c}_{\nu \eta} \\
& +\frac{1}{2} \frac{m^{2}}{n \cdot \partial} n_{\mu} \overline{\tilde{c}}_{\nu \eta}-\frac{1}{2} \frac{m^{2}}{n \cdot \partial} n_{\nu} \tilde{c}_{\eta \mu}+\frac{1}{2} \frac{m^{2}}{n \cdot \partial} n_{\nu} \overline{\tilde{c}}_{\eta \mu} \\
& -\frac{1}{2} \frac{m^{2}}{n \cdot \partial} n_{\eta} \tilde{c}_{\mu \nu}+\frac{1}{2} \frac{m^{2}}{n \cdot \partial} n_{\eta} \overline{\tilde{c}}_{\mu \nu}, \\
& s_{a b} \overline{\tilde{c}}_{\mu \nu}=\tilde{c}_{\mu \nu}^{\star}, \quad s_{a b} \tilde{c}_{\mu \nu}=\tilde{c}_{\mu \nu}^{\star}+\partial_{\mu} \tilde{\beta}_{\nu} \\
& -\partial_{\mu} \overline{\tilde{\beta}}_{\nu}-\partial_{\nu} \tilde{\beta}_{\mu}+\partial_{\nu} \overline{\tilde{\beta}}_{\mu}-\frac{1}{2} \frac{m^{2}}{n \cdot \partial} n_{\mu} \tilde{\beta}_{\nu} \\
& +\frac{1}{2} \frac{m^{2}}{n \cdot \partial} n_{\mu} \overline{\tilde{\beta}}_{v}+\frac{1}{2} \frac{m^{2}}{n \cdot \partial} n_{\nu} \tilde{\beta}_{\mu} \\
& -\frac{1}{2} \frac{m^{2}}{n \cdot \partial} n_{\nu} \overline{\tilde{\beta}}_{\mu}, \quad s_{a b} \bar{c}_{\mu \nu}=c_{\mu \nu}^{\star}, \\
& s_{a b} c_{\mu \nu}=c_{\mu \nu}^{\star}+\tilde{B}_{\mu \nu}-\overline{\tilde{B}}_{\mu \nu}, \quad s_{a b} B_{\mu \nu}=B_{\mu \nu}^{\star} \\
& +\partial_{\mu} \tilde{f}_{v}-\partial_{\mu} \overline{\tilde{f}}_{v}-\partial_{\nu} \tilde{f}_{\mu}+\partial_{\nu} \overline{\tilde{f}}_{\mu} \\
& -\frac{1}{2} \frac{m^{2}}{n \cdot \partial} n_{\mu} \tilde{f}_{\nu}+\frac{1}{2} \frac{m^{2}}{n \cdot \partial} n_{\mu} \overline{\tilde{f}}_{\nu}+\frac{1}{2} \frac{m^{2}}{n \cdot \partial} n_{\nu} \tilde{f}_{\mu} \\
& -\frac{1}{2} \frac{m^{2}}{n \cdot \partial} n_{\nu} \overline{\tilde{f}}_{\mu}, s_{a b} \phi_{\mu}=\phi_{\mu}^{\star}+\tilde{f}_{\mu}-\overline{\tilde{f}}_{\mu}, \\
& s_{a b} \bar{B}_{\mu \nu}=B_{\mu \nu}^{\star}, \quad s_{a b} \bar{\beta}_{\mu}=\beta_{\mu}^{\star} \text {, } \\
& s_{a b} \beta_{\mu}=\beta_{\mu}^{\star}+F_{\mu}-\bar{F}_{\mu}, \quad s_{a b} \overline{\tilde{\beta}}_{\mu}=\tilde{\beta}_{\mu}^{\star}, \\
& s_{a b} \tilde{\beta}_{\mu}=\tilde{\beta}_{\mu}^{\star}+\partial_{\mu} \tilde{c}_{2}-\partial_{\mu} \overline{\tilde{c}}_{2}-\frac{1}{2} \frac{m^{2}}{n \cdot \partial} n_{\mu} \tilde{c}_{2} \\
& +\frac{1}{2} \frac{m^{2}}{n \cdot \partial} n_{\mu} \overline{\tilde{c}}_{2}, \quad s_{a b} \overline{\tilde{F}}_{\mu}=\tilde{F}_{\mu}^{\star}, \\
& s_{a b} \tilde{F}_{\mu}=\tilde{F}_{\mu}^{\star}-\tilde{\partial}_{\mu} B_{2}+\tilde{\partial}_{\mu} \bar{B}_{2}+\frac{1}{2} \frac{m^{2}}{n \cdot \partial} n_{\mu} B_{2} \\
& -\frac{1}{2} \frac{m^{2}}{n \cdot \partial} n_{\mu} \bar{B}_{2}, s_{a b} \bar{f}_{\mu}=f_{\mu}^{\star},
\end{aligned}
$$

$$
\begin{aligned}
s_{a b} f_{\mu}= & f_{\mu}^{\star}-\partial_{\mu} B_{1}+\partial_{\mu} \bar{B}_{1}+\frac{1}{2} \frac{m^{2}}{n \cdot \partial} n_{\mu} B_{1} \\
& -\frac{1}{2} \frac{m^{2}}{n \cdot \partial} n_{\mu} \bar{B}_{1}, \quad s_{a b} \bar{c}_{2}=c_{2}^{\star}, \\
s_{a b} c_{2}= & c_{2}^{\star}+B-\bar{B}, \quad s_{a b} \bar{c}_{1}=c_{1}^{\star}, \\
s_{a b} c_{1}= & c_{1}^{\star}-B_{1}+\bar{B}_{1}, \quad s_{a b} \bar{\phi}_{\mu}=\phi_{\mu}^{\star}, \\
s_{a b} \overline{\tilde{c}}_{1}= & \tilde{c}_{1}^{\star}, \quad s_{a b} \tilde{c}_{1}=\tilde{c}_{1}^{\star}-B_{2}+\bar{B}_{2}, \quad s_{a b} \overline{\tilde{c}}_{2}=\tilde{c}_{2}^{\star}, \\
s_{a b} \tilde{c}_{2}= & \tilde{c}_{2}^{\star}, \quad s_{a b} \overline{\tilde{f}}_{\mu}=\tilde{f}_{\mu}^{\star}, \\
s_{a b} \tilde{f}_{\mu}= & \tilde{f}_{\mu}^{\star}, \quad s_{a b} \bar{F}_{\mu}=F_{\mu}^{\star}, \quad s_{a b} F_{\mu}=F_{\mu}^{\star}, \\
s_{a b} \bar{B}= & B^{\star}, \quad s_{a b} B=B^{\star}, \quad s_{a b} \bar{B}_{1}=B_{1}^{\star}, \\
s_{a b} B_{1}= & B_{1}^{\star}, \quad s_{a b} \bar{B}_{2}=B_{2}^{\star}, \quad s_{a b} B_{2}=B_{2}^{\star}, \\
s_{a b} \overline{\tilde{B}}_{\mu \nu}= & \tilde{B}_{\mu \nu}^{\star}, s_{a b} \tilde{B}_{\mu \nu}=\tilde{B}_{\mu \nu}^{\star} .
\end{aligned}
$$

However, the fields $B_{\mu \nu \eta}^{\star}, \tilde{c}_{\mu \nu}^{\star}, c_{\mu \nu}^{\star}, B_{\mu \nu}^{\star}, \beta_{\mu}^{\star}, \tilde{\beta}_{\mu}^{\star}, \tilde{F}_{\mu}^{\star}, f_{\mu}^{\star}, c_{2}^{\star}$, $c_{1}^{\star}, \phi_{\mu}^{\star}, \tilde{c}_{1}^{\star}, \tilde{c}_{2}^{\star}, \tilde{f}_{\mu}^{\star}, F_{\mu}^{\star}, B^{\star}, B_{1}^{\star}, B_{2}^{\star}$ and $\tilde{B}_{\mu \nu}^{\star}$, do not change under the extended anti-BRST transformation, which ensures the nilpotency of the transformation. The ghost fields associated with the shift symmetry change under the extended anti-BRST transformations as

$$
\begin{aligned}
s_{a b} L_{\mu \nu \eta} & =l_{\mu \nu \eta}, \quad s_{a b} M_{\mu \nu}=m_{\mu \nu}, \\
s_{a b} \tilde{M}_{\mu \nu} & =\bar{m}_{\mu \nu}, \quad s_{a b} N_{\mu \nu}=n_{\mu \nu}, \\
s_{a b} \tilde{N}_{\mu \nu} & =\bar{n}_{\mu \nu}, \quad s_{a b} O_{\mu}=o_{\mu}, \quad s_{a b} \tilde{O}_{\mu}=\bar{o}_{\mu}, \\
s_{a b} P_{\mu} & =p_{\mu}, \quad s_{a b} \tilde{P}_{\mu}=\bar{p}_{\mu}, \\
s_{a b} Q_{\mu} & =q_{\mu}, \quad s_{a b} \tilde{Q}_{\mu}=\bar{q}_{\mu}, \quad s_{a b} \Re=r, \\
s_{a b} \tilde{\mathfrak{R}} & =\bar{r}, \quad s_{a b} \mathfrak{S}=s, \\
s_{a b} \tilde{\mathfrak{S}} & =\bar{s}, \quad s_{a b} T_{\mu}=t_{\mu}, \quad s_{a b} \mathbb{U}=u, \\
s_{a b} \mathfrak{V} & =v, \quad s_{a b} \mathfrak{W}=w .
\end{aligned}
$$

The rest of the fields, whose transformation is written here, do not change under the extended anti-BRST transformation. To describe the superspace formulation of the Abelian 3-form gauge theory in VSR which has only extended anti-BRST invariance, we need one extra Grassmannian coordinate, say $\bar{\theta}$. It is now straightforward to define the superfields in this superspace which involve the extended anti-BRST transformations along the $\bar{\theta}$ coordinates, respectively. Therefore, the superspace description of Abelian 3-form gauge theory in VSR also holds.

\subsection{VSR-modified extended BRST and anti-BRST invariant superspace formulation}

In this section, we construct the superspace formulation for the Abelian 3-form gauge theory in VSR which is manifestly invariant under both the extended BRST transformations and the extended anti-BRST transformations. To describe such a superspace, we introduce two extra Grassmann coordinates, $\theta$ and $\bar{\theta}$ together with $x_{\mu}$. Now, we compute the components 
of the superfields by requiring the field strength to vanish along the directions of $\theta$ and $\bar{\theta}$. This leads to the superfields to have following form in this superspace generically:

$\mathfrak{T}(x, \theta, \bar{\theta})=\Phi(x)+\theta\left(s_{b} \Phi\right)+\bar{\theta}\left(s_{a b} \Phi\right)+\theta \bar{\theta}\left(s_{b} s_{a b} \Phi\right)$.

Here $\mathfrak{T}$ and $\Phi$ describe all the superfields and the fields generically. The explicit expression for the individual superfields can be found in Eq. (B5) of the Appendix B.

Exploiting the superfields (B5) we compute Eqs. (B6). Interestingly, we establish a relation between the gaugefixing Lagrangian density corresponding to shift symmetry, $\overline{\mathcal{L}}_{g f}^{B}(4.4)$, and the composite superfields as follows:

$$
\begin{aligned}
\overline{\mathcal{L}}_{g f}^{B}= & \frac{1}{2} \frac{\delta \bar{\delta}}{\delta \theta} \frac{\delta}{\delta \theta}\left[\overline{\mathcal{B}}_{\mu \nu \eta} \overline{\mathcal{B}}^{\mu \nu \eta}+2 \overline{\tilde{\mathcal{C}}}_{\mu \nu} \overline{\mathcal{C}}^{\mu \nu}+\overline{\tilde{\mathcal{B}}}_{\mu \nu} \overline{\tilde{\mathcal{B}}}^{\mu \nu}\right. \\
& +\overline{\mathcal{B}}_{\mu \nu} \overline{\mathcal{B}}^{\mu \nu}+\overline{\mathcal{B}}_{\mu} \overline{\mathcal{B}}^{\mu}+\overline{\tilde{\mathcal{B}}}_{\mu} \overline{\tilde{\mathcal{B}}}^{\mu} \\
& +2 \overline{\tilde{\mathcal{F}}}_{\mu} \overline{\mathcal{F}}^{\mu}+2 \overline{\tilde{\mathfrak{F}}}_{\mu} \overline{\mathcal{F}}^{\mu}+2 \overline{\tilde{\mathcal{C}}}_{2} \overline{\mathcal{C}}_{2}+2 \overline{\tilde{\mathcal{C}}}_{1} \overline{\mathcal{C}}_{1} \\
& \left.+\bar{\Phi}_{\mu} \bar{\Phi}^{\mu}+\overline{\mathcal{B} \mathcal{\mathcal { B }}}+\overline{\mathcal{B}}_{1} \overline{\mathcal{B}}_{1}+\overline{\mathcal{B}}_{2} \overline{\mathcal{B}}_{2}\right]
\end{aligned}
$$

This relation makes manifest that the gauge-fixed Lagrangian density $\overline{\mathcal{L}}_{g f}^{B}$ is invariant under both the extended BRST and extended anti-BRST transformations.

The super gauge-fixing fermion for the theory having both the extended BRST and extended anti-BRST invariance in superspace is given by

$\Gamma(x, \theta, \bar{\theta})=\Psi(x)+\theta s_{b} \Psi+\bar{\theta} s_{a b} \Psi+\theta \bar{\theta} s_{b} s_{a b} \Psi$.

In general, all four components of the super gauge-fixing fermion will be non-trivial, implying that if we choose as $\mathcal{L}_{g f}^{B}=s_{b} \Psi$, then it will not be invariant under generalized anti-BRST transformations. This follows from the fact that the last component of the super gauge-fixing fermion (4.35) is non-vanishing in general. However, if the gauge-fixed Lagrangian density in VSR is both extended BRST and antiBRST invariant, then the $\theta \bar{\theta}$ component of the super gaugefixing fermion would vanish, because when we use the equations of motion the bar fields vanish and the theory reduces to the original theory, where, by assumption, the gauge-fixed Lagrangian density in VSR is both extended BRST and antiBRST invariant. Therefore, for an arbitrary super gaugefixing fermion that leads to a BRST and anti-BRST invariant gauge-fixing Lagrangian density, one can choose

$\mathcal{L}_{g f}^{B}=\frac{\delta}{\delta \theta}[\delta(\bar{\theta}) \Gamma(x, \theta, \bar{\theta})]=s_{b} \Psi$.

Now, the effective Lagrangian density (4.19) possessing both the extended BRST and anti-BRST symmetries in superspace can be expressed by

$$
\begin{aligned}
\mathcal{L}_{\text {eff }}= & \mathcal{L}_{0}\left(B_{\mu \nu \rho}-\bar{B}_{\mu \nu \rho}\right)+\frac{1}{2} \frac{\delta}{\delta \theta} \frac{\delta}{\delta \theta}\left[\overline{\mathcal{B}}_{\mu \nu \eta} \overline{\mathcal{B}}^{\mu \nu \eta}\right. \\
& +2 \overline{\tilde{\mathcal{C}}}_{\mu \nu} \overline{\mathcal{C}}^{\mu \nu}+\overline{\tilde{\mathcal{B}}}_{\mu \nu} \overline{\tilde{\mathcal{B}}}^{\mu \nu}+\overline{\mathcal{B}}_{\mu \nu} \overline{\mathcal{B}}^{\mu \nu}+\overline{\mathcal{B}}_{\mu} \overline{\mathcal{B}}^{\mu} \\
& +\overline{\tilde{\mathcal{B}}}_{\mu} \overline{\tilde{\mathcal{B}}}^{\mu}+2 \overline{\tilde{\mathcal{F}}}_{\mu} \overline{\mathcal{F}}^{\mu}+2 \overline{\tilde{\tilde{F}}}_{\mu} \overline{\mathfrak{F}}^{\mu}+2 \overline{\tilde{\mathcal{C}}}_{2} \overline{\mathcal{C}}_{2}+2 \overline{\tilde{\mathcal{C}}}_{1} \overline{\mathcal{C}}_{1} \\
& \left.+\bar{\Phi}_{\mu} \bar{\Phi}^{\mu}+\overline{\mathcal{B}}^{\mathcal{B}}+\overline{\mathcal{B}}_{1} \overline{\mathcal{B}}_{1}+\overline{\mathcal{B}}_{2} \overline{\mathcal{B}}_{2}\right] \\
& +\frac{\delta}{\delta \theta}[\delta(\bar{\theta}) \Gamma(x, \theta, \bar{\theta})]
\end{aligned}
$$

Using the auxiliary fields' equations of motion the bar fields can be set zero. However, integration of the ghost fields of the shift symmetry leads to the explicit expressions for the antifields, which, when substituted into the VSR-modified Lagrangian density, yield the BV action. The superspace formulation of the BV action for the VSR-modified 3-form gauge theory has similar description as the Lorentz-invariant case.

\section{Concluding remarks}

In VSR, the spacetime translational symmetry is retained to preserve the energy-momentum and also the usual relativistic dispersion relation. Keeping the significance of VSR in mind, in this paper, we have discussed the VSR description of the non-Abelian 1-form, Abelian 2-form and Abelian 3 -form gauge theories. We have constructed the extended BRST and anti-BRST transformation (which include a shift symmetry) for these theories. To fix the shift symmetry, we need the antighost fields which coincide with the antifields of the BV formulation for each gauge theories in VSR. Furthermore, we have formulated the these VSR-modified theories in superspace. First, we have shown that the extended BRST invariant BV action of these theories in VSR can be written manifestly in a superspace with coordinates $\left(x_{\mu}, \theta\right)$ where $\theta$ is a fermionic coordinate. Similarly, extended antiBRST invariant BV action of these theories in VSR can be written manifestly in a superspace with coordinates $\left(x_{\mu}, \bar{\theta}\right)$ where $\bar{\theta}$ is another fermionic coordinate. Finally, a superspace description of the (manifestly covariant) BV action of these theories in VSR having both the extended BRST and extended anti-BRST invariance requires two Grassmann coordinates $\left(x_{\mu}, \theta, \bar{\theta}\right)$. In this context, we have noted that if the gauge-fixed Lagrangian density in VSR is both extended BRST and anti-BRST invariant, then the $\theta \bar{\theta}$ component of the super gauge-fixing fermion would vanish, because when we use the equations of motion the bar fields would vanish and the theory reduces to the original theory, where, by assumption, the gauge-fixed Lagrangian density in VSR is both extended BRST and anti-BRST invariant. The structure of the results we obtained here by studying the BV action of $p=1,2,3$-forms gauge theories with preferred direction is not very different from that of the Lorentz invariant 
case. Unlike the Lorentz-invariant case, the novel observation is that, in the VSR scenario, all the fields and superfields acquire mass, which modifies the masses of the original dispersion relations. It will be interesting to extend this superspace formulation to some regularization procedure at one-loop order, where we believe that the superfield associated with the one-loop order term of the action may have the VSR-modified anomalies and Wess-Zumino terms. Also, the extension of this superspace formulation for the more general cases in which VSR modified anomalies and Wess-Zumino terms depend on the antifields will be interesting to explore.

Acknowledgements We are thankful to Dr. Anton Ilderton for his careful reading of the manuscript and helpful comments.

Open Access This article is distributed under the terms of the Creative Commons Attribution 4.0 International License (http://creativecomm ons.org/licenses/by/4.0/), which permits unrestricted use, distribution, and reproduction in any medium, provided you give appropriate credit to the original author(s) and the source, provide a link to the Creative Commons license, and indicate if changes were made. Funded by SCOAP . $^{3}$

\section{Appendix A: Mathematical details of VSR-modified Abelian 2-form gauge theory in superspace}

The explicit component form of the superfields for VSRmodified Abelian 2-form gauge theory in superspace having both extended BRST and anti-BRST invariance are

$$
\begin{aligned}
& \mathcal{B}_{\mu \nu}(x, \theta, \bar{\theta})=B_{\mu \nu}(x)+\theta \psi_{\mu \nu}+\bar{\theta}\left(B_{\mu \nu}^{\star}+\partial_{\mu} \tilde{\rho}_{\nu}\right. \\
& -\partial_{\mu} \overline{\tilde{\rho}}_{\nu}-\partial_{\nu} \tilde{\rho}_{\mu}+\partial_{\nu} \overline{\tilde{\rho}}_{\mu}-\frac{1}{2} \frac{m^{2}}{n \cdot \partial} n_{\mu} \tilde{\rho}_{\nu} \\
& \left.+\frac{1}{2} \frac{m^{2}}{n \cdot \partial} n_{\mu} \overline{\tilde{\rho}}_{\nu}+\frac{1}{2} \frac{m^{2}}{n \cdot \partial} n_{\nu} \tilde{\rho}_{\nu}-\frac{1}{2} \frac{m^{2}}{n \cdot \partial} n_{\nu} \overline{\tilde{\rho}}_{\mu}\right) \\
& +\theta \bar{\theta}\left[L_{\mu \nu}+i\left(\partial_{\mu} \beta_{\nu}-\partial_{\nu} \beta_{\mu}\right.\right. \\
& -\partial_{\mu} \bar{\beta}_{\nu}+\partial_{\nu} \bar{\beta}_{\mu}-\frac{1}{2} \frac{m^{2}}{n \cdot \partial} n_{\mu} \beta_{\nu} \\
& \left.\left.+\frac{1}{2} \frac{m^{2}}{n \cdot \partial} n_{\nu} \beta_{\mu}+\frac{1}{2} \frac{m^{2}}{n \cdot \partial} n_{\mu} \bar{\beta}_{\nu}-\frac{1}{2} \frac{m^{2}}{n \cdot \partial} n_{\nu} \bar{\beta}_{\mu}\right)\right], \\
& \overline{\mathcal{B}}_{\mu \nu}(x, \theta, \bar{\theta})=\bar{B}_{\mu \nu}(x)+\theta\left(\psi_{\mu \nu}-\partial_{\mu} \rho_{\nu}\right. \\
& +\partial_{\mu} \bar{\rho}_{\nu}+\partial_{\nu} \rho_{\mu}-\partial_{\nu} \bar{\rho}_{\mu}+\frac{1}{2} \frac{m^{2}}{n \cdot \partial} n_{\mu} \rho_{\nu} \\
& \quad-\frac{1}{2} \frac{m^{2}}{n \cdot \partial} n_{\mu} \bar{\rho}_{\nu}-\frac{1}{2} \frac{m^{2}}{n \cdot \partial} n_{\nu} \rho_{\mu} \\
& \left.+\frac{1}{2} \frac{m^{2}}{n \cdot \partial} n_{\nu} \bar{\rho}_{\mu}\right)+\bar{\theta} B_{\mu \nu}^{\star}+\theta \bar{\theta} L_{\mu \nu}, \\
& \mathcal{M} \mu(x, \theta, \bar{\theta})=\rho_{\mu}(x)+\theta \epsilon_{\mu} \\
& +\bar{\theta}\left(\rho_{\mu}^{\star}-i \beta_{\mu}+i \bar{\beta}_{\mu}\right)+\theta \bar{\theta} M_{\mu},
\end{aligned}
$$

$$
\begin{aligned}
& \overline{\mathcal{M}}_{\mu}(x, \theta, \bar{\theta})=\bar{\rho}_{\mu}(x)+\theta\left(\epsilon_{\mu}-i \partial_{\mu} \sigma+i \partial_{\mu} \bar{\sigma}\right. \\
& \left.\quad+\frac{i}{2} \frac{m^{2}}{n \cdot \partial} n_{\mu} \sigma-\frac{i}{2} \frac{m^{2}}{n \cdot \partial} n_{\mu} \bar{\sigma}\right)+\bar{\theta} \rho_{\mu}^{\star}+\theta \bar{\theta} M_{\mu}, \\
& \mathcal{N}(x, \theta, \bar{\theta})=\sigma(x)+\theta \varepsilon+\bar{\theta}\left(\sigma^{\star}+\chi-\bar{\chi}\right)+\theta \bar{\theta} N, \\
& \overline{\mathcal{N}}(x, \theta, \bar{\theta})=\bar{\sigma}(x)+\theta \varepsilon+\bar{\theta} \sigma^{\star}+\theta \bar{\theta} N, \\
& \tilde{\mathcal{M}}_{\mu}(x, \theta, \bar{\theta})=\tilde{\rho}_{\mu}(x)+\theta \xi_{\mu}+\bar{\theta}\left(\tilde{\rho}_{\mu}^{\star}-i \partial_{\mu} \tilde{\sigma}\right. \\
& \left.\quad+\frac{i}{2} \frac{m^{2}}{n \cdot \partial} n_{\mu} \tilde{\sigma}+i \partial_{\mu} \overline{\tilde{\sigma}}-\frac{i}{2} \frac{m^{2}}{n \cdot \partial} n_{\mu} \overline{\tilde{\sigma}}\right) \\
& \quad+\theta \bar{\theta}\left(\bar{M} \bar{M}_{\mu}-i \partial_{\mu} \tilde{\chi}+\frac{i}{2} \frac{m^{2}}{n \cdot \partial} n_{\mu} \tilde{\chi}\right. \\
& \left.\quad+i \partial_{\mu} \overline{\tilde{\chi}}-\frac{i}{2} \frac{m^{2}}{n \cdot \partial} n_{\mu} \overline{\tilde{\chi}}\right), \\
& \overline{\tilde{\mathcal{M}}}{ }_{\mu}(x, \theta, \bar{\theta})=\overline{\tilde{\rho}}_{\mu}(x)+\theta\left(\xi_{\mu}-i \beta_{\mu}+i \bar{\beta}_{\mu}\right)+\bar{\theta} \tilde{\rho}_{\mu}^{\star}+\theta \bar{\theta} \bar{M}_{\mu}, \\
& \mathcal{S}_{\mu}(x, \theta, \bar{\theta})=\beta_{\mu}(x)+\theta \eta_{\mu}+\bar{\theta} \beta_{\mu}^{\star}+\theta \bar{\theta} S_{\mu}, \\
& \overline{\mathcal{S}}(x, \theta, \bar{\theta})=\bar{\beta} \bar{\beta}_{\mu}(x)+\theta \eta_{\mu}+\bar{\theta} \beta_{\mu}^{\star}+\theta \bar{\theta} S_{\mu}, \\
& \tilde{\mathcal{N}}(x, \theta, \bar{\theta})=\tilde{\sigma}(x)+\theta \psi+\bar{\theta} \tilde{\sigma}^{\star}+\theta \bar{\theta} \bar{N}, \\
& \overline{\tilde{\mathcal{N}}}(x, \theta, \bar{\theta})=\tilde{\tilde{\sigma}}(x)+\theta(\psi-\tilde{\chi}+\overline{\tilde{\psi}})+\bar{\theta} \tilde{\sigma}^{\star}+\theta \bar{\theta} \bar{N}, \\
& \mathcal{O}(x, \theta, \bar{\theta})=\chi(x)+\theta \Sigma+\bar{\theta} \chi^{\star}+\theta \bar{\theta} O, \\
& \overline{\mathcal{O}}(x, \theta, \bar{\theta})=\bar{\chi}(x)+\theta \Sigma+\bar{\theta} \chi^{\star}+\theta \bar{\theta} O, \\
& \tilde{\mathcal{O}}(x, \theta, \bar{\theta})=\tilde{\chi}(x)+\theta \eta+\bar{\theta} \tilde{\chi}^{\star}+\theta \bar{\theta} \bar{O}, \\
& \overline{\tilde{\mathcal{O}}}(x, \theta, \bar{\theta})=\overline{\tilde{\chi}}(x)+\theta \eta+\bar{\theta} \tilde{\chi}^{\star}+\theta \bar{\theta} \bar{O}, \\
& \mathcal{T}(x, \theta, \bar{\theta})=\varphi(x)+\theta \phi+\bar{\theta}\left(\varphi^{\star}-\tilde{\chi}+\overline{\tilde{\chi}}\right)+\theta \bar{\theta} T, \\
& \overline{\mathcal{T}}(x, \theta, \bar{\theta})=\bar{\varphi}(x)+\theta(\phi-\chi+\bar{\chi})+\bar{\theta} \varphi^{\star}+\theta \bar{\theta} T .
\end{aligned}
$$

\section{Appendix B: Mathematical details of VSR-modified Abelian 3-form gauge theory}

The explicit form of the BV action of VSR-modified Abelian 3 -form gauge theory is calculated as

$$
\begin{aligned}
\mathcal{L}_{\text {eff }}= & \frac{1}{24} H_{\mu \nu \eta \xi} H^{\mu \nu \eta \xi}+B_{\mu \nu \eta}^{\star}\left(\partial^{\mu} c^{\nu \eta}+\partial^{\nu} c^{\eta \mu}\right. \\
& +\partial^{\eta} c^{\mu \nu}-\frac{1}{2} \frac{m^{2}}{n \cdot \partial} n^{\mu} c^{\nu \eta}-\frac{1}{2} \frac{m^{2}}{n \cdot \partial} n^{\nu} c^{\eta \mu} \\
& \left.-\frac{1}{2} \frac{m^{2}}{n \cdot \partial} n^{\eta} c^{\mu \nu}\right)-\tilde{c}_{\mu \nu}^{\star}\left(\partial^{\mu} \beta^{\nu}-\partial^{\nu} \beta^{\mu}-\frac{1}{2} \frac{m^{2}}{n \cdot \partial} n^{\mu} \beta^{\nu}\right. \\
& \left.+\frac{1}{2} \frac{m^{2}}{n \cdot \partial} n^{\nu} \beta^{\mu}\right)-c_{\mu \nu}^{\star} B^{\mu \nu} \\
& +\tilde{B}_{\mu \nu}^{\star}\left(\partial^{\mu} f^{\nu}-\partial^{\nu} f^{\mu}-\frac{1}{2} \frac{m^{2}}{n \cdot \partial} n^{\mu} f^{\nu}\right. \\
& \left.+\frac{1}{2} \frac{m^{2}}{n \cdot \partial} n^{\nu} f^{\mu}\right)+\beta_{\mu}^{\star} \partial^{\mu} c_{2}+\tilde{\beta}_{\mu}^{\star} \tilde{F}^{\mu}+\tilde{F}_{\mu}^{\star} \partial^{\mu} B \\
& -f_{\mu}^{\star} \partial^{\mu} B_{1}-\beta_{\mu}^{\star} \frac{1}{2} \frac{m^{2}}{n \cdot \partial} n^{\mu} c_{2}-\tilde{F}_{\mu}^{\star} \frac{1}{2} \frac{m^{2}}{n \cdot \partial} n^{\mu} B \\
& +f_{\mu}^{\star} \frac{1}{2} \frac{m^{2}}{n \cdot \partial} n^{\mu} B_{1}-c_{2}^{\star} B_{2}+\tilde{c}_{1}^{\star} B
\end{aligned}
$$




$$
\begin{aligned}
& -c_{1}^{\star} B_{1}+\phi_{\mu}^{\star} f^{\mu}-\left(B^{\mu \nu \eta \star}+\frac{\delta \Psi}{\delta B_{\mu \nu \eta}}\right) L_{\mu \nu \eta} \\
& +\left(\tilde{c}^{\mu \nu \star}+\frac{\delta \Psi}{\delta c_{\mu \nu}}\right) M_{\mu \nu} \\
& +\left(c^{\mu \nu \star}+\frac{\delta \Psi}{\delta \tilde{c}_{\mu \nu}}\right) \tilde{M}_{\mu \nu}-\left(B^{\mu \nu \star}+\frac{\delta \Psi}{\delta B_{\mu \nu}}\right) N_{\mu \nu} \\
& -\left(\tilde{B}^{\mu \nu \star}+\frac{\delta \Psi}{\delta \tilde{B}_{\mu \nu}}\right) \tilde{N}_{\mu \nu} \\
& -\left(\beta^{\mu \star}+\frac{\delta \Psi}{\delta \beta_{\mu}}\right) O_{\mu}-\left(\tilde{\beta}^{\mu \nu \star}+\frac{\delta \Psi}{\delta \tilde{\beta}_{\mu}}\right) \tilde{O}_{\mu} \\
& +\left(\tilde{F}^{\mu \star}+\frac{\delta \Psi}{\delta F_{\mu}}\right) P_{\mu} \\
& +\left(F^{\mu \star}+\frac{\delta \Psi}{\delta \tilde{F}_{\mu}}\right) \tilde{P}_{\mu}+\left(\tilde{f}^{\mu \star}+\frac{\delta \Psi}{\delta f_{\mu}}\right) Q_{\mu} \\
& +\left(f^{\mu \star}+\frac{\delta \Psi}{\delta \tilde{f}_{\mu}}\right) \tilde{Q}_{\mu}+\left(\tilde{c}_{2}^{\star}+\frac{\delta \Psi}{\delta c_{2}}\right) \Re \\
& +\left(c_{2}^{\star}+\frac{\delta \Psi}{\delta \tilde{c}_{2}}\right) \tilde{\mathfrak{R}}+\left(\tilde{c}_{1}^{\star}+\frac{\delta \Psi}{\delta c_{1}}\right) \mathfrak{S} \\
& +\left(c_{1}^{\star}+\frac{\delta \Psi}{\delta \tilde{c}_{1}}\right) \tilde{\mathfrak{S}}-\left(\phi^{\mu \star}+\frac{\delta \Psi}{\delta \phi_{\mu}}\right) T_{\mu} \\
& -\left(B^{\star}+\frac{\delta \Psi}{\delta B}\right) \mathbb{U}-\left(B_{1}^{\star}+\frac{\delta \Psi}{\delta B_{1}}\right) \mathfrak{V} \\
& -\left(B_{2}^{\star}+\frac{\delta \Psi}{\delta B_{2}}\right) \mathfrak{W} \text {. }
\end{aligned}
$$

The explicit form of the VSR-modified extended BRST transformations for the Abelian 3-form gauge theory is

$$
\begin{aligned}
& s_{b} B_{\mu \nu \eta}=L_{\mu \nu \eta}, \\
& s_{b} \bar{B}_{\mu \nu \eta}=L_{\mu \nu \eta}-\left(\partial_{\mu} c_{\nu \eta}-\partial_{\mu} \bar{c}_{\nu \eta}+\partial_{\nu} c_{\eta \mu}-\partial_{\nu} \bar{c}_{\eta \mu}\right. \\
& +\partial_{\eta} c_{\mu \nu}-\partial_{\eta} \bar{c}_{\mu \nu}-\frac{1}{2} \frac{m^{2}}{n \cdot \partial} n_{\mu} c_{\nu \eta} \\
& +\frac{1}{2} \frac{m^{2}}{n \cdot \partial} n_{\mu} \bar{c}_{\nu \eta}-\frac{1}{2} \frac{m^{2}}{n \cdot \partial} n_{\nu} c_{\eta \mu}+\frac{1}{2} \frac{m^{2}}{n \cdot \partial} n_{\nu} \bar{c}_{\eta \mu} \\
& \left.-\frac{1}{2} \frac{m^{2}}{n \cdot \partial} n_{\eta} c_{\mu \nu}+\frac{1}{2} \frac{m^{2}}{n \cdot \partial} n_{\eta} \bar{c}_{\mu \nu}\right) \text {, } \\
& s_{b} c_{\mu \nu}=M_{\mu \nu}, s_{b} \tilde{c}_{\mu \nu}=\tilde{M}_{\mu \nu}, s_{b} \bar{c}_{\mu \nu}=M_{\mu \nu} \\
& -\left(\partial_{\mu} \beta_{v}-\partial_{\mu} \bar{\beta}_{v}-\partial_{\nu} \beta_{\mu}+\partial_{\nu} \bar{\beta}_{\mu}\right. \\
& -\frac{1}{2} \frac{m^{2}}{n \cdot \partial} n_{\mu} \beta_{v}+\frac{1}{2} \frac{m^{2}}{n \cdot \partial} n_{\mu} \bar{\beta}_{v} \\
& \left.+\frac{1}{2} \frac{m^{2}}{n \cdot \partial} n_{\nu} \beta_{\mu}-\frac{1}{2} \frac{m^{2}}{n \cdot \partial} n_{\nu} \bar{\beta}_{\mu}\right) \text {, } \\
& s_{b} \overline{\tilde{c}}_{\mu \nu}=\tilde{M}_{\mu \nu}-B_{\mu \nu}+\bar{B}_{\mu \nu}, \quad s_{b} B_{\mu \nu}=N_{\mu \nu}, \\
& s_{b} \bar{B}_{\mu \nu}=N_{\mu \nu}, \quad s_{b} \tilde{B}_{\mu \nu}=\tilde{N}_{\mu \nu} \\
& s_{b} \overline{\tilde{B}}_{\mu \nu}=\tilde{N}_{\mu \nu}-\left(\partial_{\mu} f_{\nu}-\partial_{\mu} \bar{f}_{\nu}-\partial_{\nu} f_{\mu}+\partial_{\nu} \bar{f}_{\mu}\right.
\end{aligned}
$$

$$
\begin{aligned}
& -\frac{1}{2} \frac{m^{2}}{n \cdot \partial} n_{\mu} f_{v}+\frac{1}{2} \frac{m^{2}}{n \cdot \partial} n_{\mu} \bar{f}_{v} \\
& \left.+\frac{1}{2} \frac{m^{2}}{n \cdot \partial} n_{\nu} f_{\mu}-\frac{1}{2} \frac{m^{2}}{n \cdot \partial} n_{\nu} \bar{f}_{\mu}\right), \\
& s_{b} \beta_{\mu}=O_{\mu}, s_{b} \bar{\beta}_{\mu}=O_{\mu}-\partial_{\mu} c_{2}+\partial_{\mu} \bar{c}_{2} \\
& +\frac{1}{2} \frac{m^{2}}{n \cdot \partial} n_{\mu} c_{2}-\frac{1}{2} \frac{m^{2}}{n \cdot \partial} n_{\mu} \bar{c}_{2}, \\
& s_{b} \tilde{\beta}_{\mu}=\tilde{O}_{\mu}, \quad s_{b} \overline{\tilde{\beta}}_{\mu}=\tilde{O}_{\mu}-\tilde{F}_{\mu}+\overline{\tilde{F}}_{\mu}, s_{b} F_{\mu}=P_{\mu}, \\
& s_{b} \bar{F}_{\mu}=P_{\mu}+\partial_{\mu} B-\partial_{\mu} \bar{B}-\frac{1}{2} \frac{m^{2}}{n \cdot \partial} n_{\mu} B+\frac{1}{2} \frac{m^{2}}{n \cdot \partial} n_{\mu} \bar{B}, \\
& s_{b} \tilde{F}_{\mu}=\tilde{P}_{\mu}, \quad s_{b} \overline{\tilde{F}}_{\mu}=\tilde{P}_{\mu}, \quad s_{b} f_{\mu}=Q_{\mu}, \\
& s_{b} \bar{f}_{\mu}=Q_{\mu}, s_{b} \tilde{f}_{\mu}=\tilde{Q}_{\mu}, \\
& s_{b} \overline{\tilde{f}}_{\mu}=\tilde{Q}_{\mu}-\partial_{\mu} B_{1}+\partial_{\mu} \bar{B}_{1} \\
& -\frac{1}{2} \frac{m^{2}}{n \cdot \partial} n_{\mu} B_{1}+\frac{1}{2} \frac{m^{2}}{n \cdot \partial} n_{\mu} \bar{B}_{1}, \\
& s_{b} c_{2}=\Re, \quad s_{b} \bar{c}_{2}=\Re, s_{b} \tilde{c}_{2}=\tilde{\Re}, \\
& s_{b} \overline{\tilde{c}}_{2}=\tilde{\Re}-B_{2}+\bar{B}_{2} \text {, } \\
& s_{b} c_{1}=\mathfrak{S}, \quad s_{b} \bar{c}_{1}=\mathfrak{S}+B-\bar{B}, \quad s_{b} \tilde{c}_{1}=\tilde{\mathfrak{S}}, \\
& s_{b} \phi_{\mu}=T_{\mu}, \quad s_{b} \bar{\phi}_{\mu}=T_{\mu}-f_{\mu}+\bar{f}_{\mu}, \\
& s_{b} \overline{\tilde{c}}_{1}=\tilde{\mathfrak{S}}-B_{1}+\bar{B}_{1}, s_{b} B=\mathbb{U}, s_{b} B_{1}=\mathfrak{V}, \quad s_{b} \bar{B}_{1}=\mathfrak{V}, \\
& s_{b} \bar{B}=\mathbb{U}, s_{b} B_{2}=\mathfrak{W}, \quad s_{b} \bar{B}_{2}=\mathfrak{W}, \quad s_{b} \Omega=0,
\end{aligned}
$$

where $\Omega \equiv\left[L_{\mu \nu \eta}, M_{\mu \nu}, \tilde{M}_{\mu \nu}, N_{\mu \nu}, \tilde{N}_{\mu \nu}, O_{\mu}, \tilde{O}_{\mu}, P_{\mu}\right.$, $\left.\tilde{P}_{\mu}, Q_{\mu}, \tilde{Q}_{\mu}, \mathfrak{R}, \tilde{\mathfrak{R}}, \mathfrak{S}, \tilde{\mathfrak{S}}, T_{\mu}, \mathbb{U}, \mathfrak{V}, \mathfrak{W}\right]$ are the ghosts corresponding to the shift symmetry.

The VSR-modified BRST transformations of the antifields are

$$
\begin{aligned}
& s_{b} B_{\mu \nu \eta}^{\star}=l_{\mu \nu \eta}, \quad s_{b} c_{\mu \nu}^{\star}=m_{\mu \nu}, \\
& s_{b} \tilde{c}_{\mu \nu}^{\star}=\bar{m}_{\mu \nu}, \quad s_{b} B_{\mu \nu}^{\star}=n_{\mu \nu}, \\
& s_{b} \beta_{\mu}^{\star}=o_{\mu}, \quad s_{b} \tilde{\beta}_{\mu}^{\star}=\bar{o}_{\mu}, \quad s_{b} F_{\mu}^{\star}=p_{\mu}, \\
& s_{b}{\tilde{F_{\mu}}}^{\star}=\bar{p}_{\mu}, \quad s_{b} f_{\mu}^{\star}=q_{\mu}, \\
& s_{b}{\tilde{f_{\mu}}}^{\star}=\bar{q}_{\mu}, \quad s_{b} c_{2}^{\star}=r, \quad s_{b}{\tilde{c_{2}}}^{\star}=\bar{r}, \\
& s_{b} c_{1}^{\star}=s, \quad s_{b}{\tilde{c_{1}}}^{\star}=\bar{s}, \\
& s_{b} \phi_{\mu}^{\star}=t_{\mu}, \quad s_{b} B^{\star}=u, \quad s_{b} B_{1}^{\star}=v, \\
& s_{b} B_{2}^{\star}=w, \quad s_{b} \tilde{B}_{\mu \nu}^{\star}=\bar{n}_{\mu \nu}, \quad s_{b} \Lambda=0 .
\end{aligned}
$$

where $\Lambda \equiv l_{\mu \nu \eta}, m_{\mu \nu}, \bar{m}_{\mu \nu}, n_{\mu \nu}, \bar{n}_{\mu \nu}, o_{\mu}, \bar{o}_{\mu}, p_{\mu}, \bar{p}_{\mu}, q_{\mu}$, $\bar{q}_{\mu}, r, \bar{r}, s, \bar{s}, t_{\mu}, u, v, w$ are the auxiliary fields.

The superfields and anti-superfields in component form for the VSR modified extended BRST invariant 3-form theory are

$$
\begin{aligned}
\mathcal{B}_{\mu \nu \eta}(x, \theta)= & B_{\mu \nu \eta}(x)+\theta L_{\mu \nu \eta}, \mathcal{C}_{\mu \nu}(x, \theta)=c_{\mu \nu}(x)+\theta M_{\mu \nu}, \\
\overline{\mathcal{B}}_{\mu \nu \eta}(x, \theta)= & \bar{B}_{\mu \nu \eta}(x)+\theta\left[L_{\mu \nu \eta}-\left(\partial_{\mu} c_{\nu \eta}-\partial_{\mu} \bar{c}_{\nu \eta}\right.\right. \\
& +\partial_{\nu} c_{\eta \mu}-\partial_{\nu} \bar{c}_{\eta \mu}+\partial_{\eta} c_{\mu \nu}-\partial_{\eta} \bar{c}_{\mu \nu} \\
& -\frac{1}{2} \frac{m^{2}}{n \cdot \partial} n_{\mu} c_{\nu \eta}+\frac{1}{2} \frac{m^{2}}{n \cdot \partial} n_{\mu} \bar{c}_{\nu \eta}-\frac{1}{2} \frac{m^{2}}{n \cdot \partial} n_{\nu} c_{\eta \mu}
\end{aligned}
$$




$$
\begin{aligned}
& +\frac{1}{2} \frac{m^{2}}{n \cdot \partial} n_{\nu} \bar{c}_{\eta \mu}-\frac{1}{2} \frac{m^{2}}{n \cdot \partial} n_{\eta} c_{\mu \nu} \\
& \left.\left.+\frac{1}{2} \frac{m^{2}}{n \cdot \partial} n_{\eta} \bar{c}_{\mu \nu}\right)\right] \\
& \overline{\mathcal{C}}_{\mu \nu}(x, \theta)=\bar{c}_{\mu \nu}(x)+\theta\left[M_{\mu \nu}-\left(\partial_{\mu} \beta_{v}-\partial_{\mu} \bar{\beta}_{\nu}\right.\right. \\
& -\partial_{\nu} \beta_{\mu}+\partial_{\nu} \bar{\beta}_{\mu}-\frac{1}{2} \frac{m^{2}}{n \cdot \partial} n_{\mu} \beta_{\nu} \\
& \left.\left.+\frac{1}{2} \frac{m^{2}}{n \cdot \partial} n_{\mu} \bar{\beta}_{\nu}+\frac{1}{2} \frac{m^{2}}{n \cdot \partial} n_{\nu} \beta_{\mu}-\frac{1}{2} \frac{m^{2}}{n \cdot \partial} n_{\nu} \bar{\beta}_{\mu}\right)\right] \text {, } \\
& \tilde{\mathcal{C}}_{\mu \nu}(x, \theta)=\tilde{c}_{\mu \nu}(x)+\theta \tilde{M}_{\mu \nu}, \overline{\tilde{\mathcal{C}}}_{\mu \nu}(x, \theta) \\
& =\overline{\tilde{c}}_{\mu \nu}(x)+\theta\left(\tilde{M}_{\mu \nu}-B_{\mu \nu}+\bar{B}_{\mu \nu}\right), \\
& \mathcal{B}_{\mu \nu}(x, \theta)=B_{\mu \nu}(x)+\theta N_{\mu \nu}, \quad \overline{\mathcal{B}}_{\mu \nu}(x, \theta)=\bar{B}_{\mu \nu}(x)+\theta N_{\mu \nu}, \\
& \tilde{\mathcal{B}}_{\mu \nu}(x, \theta)=\tilde{B}_{\mu \nu}(x)+\theta \tilde{N}_{\mu \nu}, \mathcal{B}_{\mu}(x, \theta)=\beta_{\mu}(x)+\theta O_{\mu}, \\
& \overline{\tilde{\mathcal{B}}}_{\mu \nu}(x, \theta)=\overline{\tilde{B}}_{\mu \nu}(x)+\theta\left[\tilde{N}_{\mu \nu}-\left(\partial_{\mu} f_{\nu}-\partial_{\mu} \bar{f}_{\nu}-\partial_{\nu} f_{\mu}+\partial_{\nu} \bar{f}_{\mu}\right.\right. \\
& -\frac{1}{2} \frac{m^{2}}{n \cdot \partial} n_{\mu} f_{v}+\frac{1}{2} \frac{m^{2}}{n \cdot \partial} n_{\mu} \bar{f}_{v} \\
& \left.\left.+\frac{1}{2} \frac{m^{2}}{n \cdot \partial} n_{\nu} f_{\mu}-\frac{1}{2} \frac{m^{2}}{n \cdot \partial} n_{\nu} \bar{f}_{\mu}\right)\right] \text {, } \\
& \overline{\mathcal{B}}_{\mu}(x, \theta)=\bar{\beta}_{\mu}(x)+\theta\left(O_{\mu}-\partial_{\mu} c_{2}+\partial_{\mu} \bar{c}_{2}\right. \\
& \left.+\frac{1}{2} \frac{m^{2}}{n \cdot \partial} n_{\mu} c_{2}-\frac{1}{2} \frac{m^{2}}{n \cdot \partial} n_{\mu} \bar{c}_{2}\right), \\
& \tilde{\mathcal{B}}_{\mu}(x, \theta)=\tilde{\beta}_{\mu}(x)+\theta \tilde{O}_{\mu}, \overline{\tilde{\mathcal{B}}}_{\mu}(x, \theta)=\overline{\tilde{\beta}}_{\mu}(x) \\
& +\theta\left(\tilde{O}_{\mu}-\tilde{F}_{\mu}+\overline{\tilde{F}}_{\mu}\right) \text {, } \\
& \overline{\mathcal{F}}_{\mu}(x, \theta)=\bar{F}_{\mu}(x)+\theta\left(P_{\mu}+\partial_{\mu} B-\partial_{\mu} \bar{B}\right. \\
& \left.-\frac{1}{2} \frac{m^{2}}{n \cdot \partial} n_{\mu} B+\frac{1}{2} \frac{m^{2}}{n \cdot \partial} n_{\mu} B\right), \\
& \tilde{\mathcal{F}}_{\mu}(x, \theta)=\tilde{F}_{\mu}(x)+\theta \tilde{P}_{\mu}, \overline{\tilde{\mathcal{F}}}_{\mu}(x, \theta)=\overline{\tilde{F}}_{\mu}(x) \\
& +\theta \tilde{P}_{\mu}, \quad \mathcal{F}_{\mu}(x, \theta)=F_{\mu}(x)+\theta P_{\mu}, \\
& \mathfrak{F}_{\mu}(x, \theta)=f_{\mu}(x)+\theta Q_{\mu}, \quad \overline{\mathfrak{F}}_{\mu}(x, \theta)=\bar{f}_{\mu}(x) \\
& +\theta Q_{\mu}, \quad \tilde{\mathfrak{F}}_{\mu}(x, \theta)=\tilde{f}_{\mu}(x)+\theta \tilde{Q}_{\mu}, \\
& \overline{\tilde{\mathfrak{F}}}_{\mu}(x, \theta)=\overline{\tilde{f}}_{\mu}(x)+\theta\left(\tilde{Q}_{\mu}-\partial_{\mu} B_{1}+\partial_{\mu} \bar{B}_{1}\right. \\
& \left.+\frac{1}{2} \frac{m^{2}}{n \cdot \partial} n_{\mu} B_{1}-\frac{1}{2} \frac{m^{2}}{n \cdot \partial} n_{\mu} \bar{B}_{1}\right), \\
& \mathcal{C}_{2}(x, \theta)=c_{2}(x)+\theta \Re, \quad \overline{\mathcal{C}}_{2}(x, \theta)=\bar{c}_{2}(x)+\theta \Re, \\
& \tilde{\mathcal{C}}_{2}(x, \theta)=\tilde{c}_{2}(x)+\theta \tilde{\Re}, \\
& \overline{\tilde{\mathcal{C}}}_{2}(x, \theta)=\overline{\tilde{c}}_{2}(x)+\theta\left(\tilde{\mathfrak{R}}-B_{2}+\bar{B}_{2}\right), \\
& \mathcal{C}_{1}(x, \theta)=c_{1}(x)+\theta \mathfrak{S}, \\
& \tilde{\mathcal{C}}_{1}(x, \theta)=\tilde{c}_{1}(x)+\theta \tilde{\mathfrak{S}}, \overline{\tilde{\mathcal{C}}}_{1}(x, \theta) \\
& =\overline{\tilde{c}}_{1}(x)+\theta\left(\tilde{\mathfrak{S}}-B_{1}+\bar{B}_{1}\right), \\
& \Phi_{\mu}(x, \theta)=\phi_{\mu}(x)+\theta T_{\mu}, \bar{\Phi}_{\mu}(x, \theta) \\
& =\bar{\phi}_{\mu}(x)+\theta\left(T_{\mu}-f_{\mu}+\bar{f}_{\mu}\right) \text {, } \\
& =\bar{B}(x)+\theta \mathbb{U}, \quad \mathcal{B}_{1}(x, \theta)=B_{1}(x)+\theta \mathfrak{V}, \\
& \mathcal{B}_{\mu \nu \eta}(x, \theta, \bar{\theta})=B_{\mu \nu \eta}(x)+\theta L_{\mu \nu \eta}+\bar{\theta}\left(B_{\mu \nu \eta}^{\star}+\partial_{\mu} \tilde{c}_{\nu \eta}\right. \\
& -\partial_{\mu} \overline{\tilde{c}}_{\nu \eta}+\partial_{\nu} \tilde{c}_{\eta \mu}-\partial_{\nu} \overline{\tilde{c}}_{\eta \mu}+\partial_{\eta} \tilde{c}_{\mu \nu} \\
& -\partial_{\eta} \overline{\tilde{c}}_{\mu \nu}-\frac{1}{2} \frac{m^{2}}{n \cdot \partial} n_{\mu} \tilde{c}_{\nu \eta}+\frac{1}{2} \frac{m^{2}}{n \cdot \partial} n_{\mu} \overline{\tilde{c}}_{\nu \eta} \\
& -\frac{1}{2} \frac{m^{2}}{n \cdot \partial} n_{\nu} \tilde{c}_{\eta \mu}+\frac{1}{2} \frac{m^{2}}{n \cdot \partial} n_{\nu} \overline{\tilde{c}}_{\eta \mu} \\
& \left.-\frac{1}{2} \frac{m^{2}}{n \cdot \partial} n_{\eta} \tilde{c}_{\mu \nu}+\frac{1}{2} \frac{m^{2}}{n \cdot \partial} n_{\eta} \overline{\tilde{c}}_{\mu \nu}\right)+\theta \bar{\theta}\left(l_{\mu \nu \eta}\right. \\
& +\partial_{\mu} B_{\nu \eta}-\partial_{\mu} \bar{B}_{\nu \eta}+\partial_{\nu} B_{\eta \mu} \\
& -\partial_{\nu} \bar{B}_{\eta \mu}+\partial_{\eta} B_{\mu \nu}-\partial_{\eta} \bar{B}_{\mu \nu} \\
& -\frac{1}{2} \frac{m^{2}}{n \cdot \partial} n_{\mu} B_{v \eta}+\frac{1}{2} \frac{m^{2}}{n \cdot \partial} n_{\mu} \bar{B}_{v \eta} \\
& -\frac{1}{2} \frac{m^{2}}{n \cdot \partial} n_{\nu} B_{\eta \mu}+\frac{1}{2} \frac{m^{2}}{n \cdot \partial} n_{\nu} \bar{B}_{\eta \mu} \\
& \left.-\frac{1}{2} \frac{m^{2}}{n \cdot \partial} n_{\eta} B_{\mu \nu}+\frac{1}{2} \frac{m^{2}}{n \cdot \partial} n_{\eta} \bar{B}_{\mu \nu}\right), \\
& \mathcal{C}_{\mu \nu}(x, \theta, \bar{\theta})=c_{\mu \nu}(x)+\theta M_{\mu \nu}+\bar{\theta}\left(c_{\mu \nu}^{\star}+\tilde{B}_{\mu \nu}-\overline{\tilde{B}}_{\mu \nu}\right) \\
& +\theta \bar{\theta}\left(m_{\mu \nu}+\partial_{\mu} f_{v}-\partial_{\mu} \bar{f}_{\nu}-\partial_{\nu} f_{\mu}\right. \\
& +\partial_{\nu} \bar{f}_{\mu}-\frac{1}{2} \frac{m^{2}}{n \cdot \partial} n_{\mu} f_{\nu}+\frac{1}{2} \frac{m^{2}}{n \cdot \partial} n_{\mu} \bar{f}_{\nu} \\
& \left.+\frac{1}{2} \frac{m^{2}}{n \cdot \partial} n_{\nu} f_{\mu}-\frac{1}{2} \frac{m^{2}}{n \cdot \partial} n_{v} \bar{f}_{\mu}\right)
\end{aligned}
$$




$$
\begin{aligned}
& \overline{\mathcal{B}}_{\mu \nu \eta}(x, \theta, \bar{\theta})=\bar{B}_{\mu \nu \eta}(x)+\theta\left(L_{\mu \nu \eta}-\left(\partial_{\mu} c_{\nu \eta}-\partial_{\mu} \bar{c}_{\nu \eta}\right.\right. \\
& \left.\left.+\frac{1}{2} \frac{m^{2}}{n \cdot \partial} n_{\nu} f_{\mu}-\frac{1}{2} \frac{m^{2}}{n \cdot \partial} n_{\nu} \bar{f}_{\mu}\right)\right)+\bar{\theta} \tilde{B}_{\mu \nu}^{\star}+\theta \bar{\theta} \bar{n}_{\mu \nu}, \\
& +\partial_{\nu} c_{\eta \mu}-\partial_{\nu} \bar{c}_{\eta \mu}+\partial_{\eta} c_{\mu \nu}-\partial_{\eta} \bar{c}_{\mu \nu} \\
& -\frac{1}{2} \frac{m^{2}}{n \cdot \partial} n_{\mu} c_{\nu \eta}+\frac{1}{2} \frac{m^{2}}{n \cdot \partial} n_{\mu} \bar{c}_{\nu \eta} \\
& -\frac{1}{2} \frac{m^{2}}{n \cdot \partial} n_{\nu} c_{\eta \mu}+\frac{1}{2} \frac{m^{2}}{n \cdot \partial} n_{\nu} \bar{c}_{\eta \mu}-\frac{1}{2} \frac{m^{2}}{n \cdot \partial} n_{\eta} c_{\mu \nu} \\
& \left.\left.+\frac{1}{2} \frac{m^{2}}{n \cdot \partial} n_{\eta} \bar{c}_{\mu \nu}\right)\right)+\bar{\theta} B_{\mu \nu \eta}^{\star}+\theta \bar{\theta} l_{\mu \nu \eta}, \\
& \overline{\mathcal{C}}_{\mu \nu}(x, \theta, \bar{\theta})=\bar{c}_{\mu \nu}(x)+\theta\left(M_{\mu \nu}-\left(\partial_{\mu} \beta_{\nu}-\partial_{\mu} \bar{\beta}_{\nu}-\partial_{\nu} \beta_{\mu}\right.\right. \\
& +\partial_{\nu} \bar{\beta}_{\mu}-\frac{1}{2} \frac{m^{2}}{n \cdot \partial} n_{\mu} \beta_{v}+\frac{1}{2} \frac{m^{2}}{n \cdot \partial} n_{\mu} \bar{\beta}_{v} \\
& \left.\left.+\frac{1}{2} \frac{m^{2}}{n \cdot \partial} n_{\nu} \beta_{\mu}-\frac{1}{2} \frac{m^{2}}{n \cdot \partial} n_{\nu} \bar{\beta}_{\mu}\right)\right)+\bar{\theta} c_{\mu \nu}^{\star}+\theta \bar{\theta} m_{\mu \nu}, \\
& \tilde{\mathcal{C}}_{\mu \nu}(x, \theta, \bar{\theta})=\tilde{c}_{\mu \nu}(x)+\theta \tilde{M}_{\mu \nu}+\bar{\theta}\left(\tilde{c}_{\mu \nu}^{\star}+\partial_{\mu} \tilde{\beta}_{\nu}\right. \\
& -\partial_{\mu} \overline{\tilde{\beta}}_{v}-\partial_{\nu} \tilde{\beta}_{\mu}+\partial_{\nu} \overline{\tilde{\beta}}_{\mu}-\frac{1}{2} \frac{m^{2}}{n \cdot \partial} n_{\mu} \tilde{\beta}_{v} \\
& \left.+\frac{1}{2} \frac{m^{2}}{n \cdot \partial} n_{\mu} \overline{\tilde{\beta}}_{\nu}+\frac{1}{2} \frac{m^{2}}{n \cdot \partial} n_{\nu} \tilde{\beta}_{\mu}-\frac{1}{2} \frac{m^{2}}{n \cdot \partial} n_{\nu} \overline{\tilde{\beta}}_{\mu}\right) \\
& +\theta \bar{\theta}\left(\bar{m}_{\mu v}+\partial_{\mu} \tilde{F}_{v}-\partial_{\mu} \overline{\tilde{F}}_{v}\right. \\
& -\partial_{\nu} \tilde{F}_{\mu}+\partial_{\nu} \overline{\tilde{F}}_{\mu}-\frac{1}{2} \frac{m^{2}}{n \cdot \partial} n_{\mu} \tilde{F}_{v}+\frac{1}{2} \frac{m^{2}}{n \cdot \partial} n_{\mu} \overline{\tilde{F}}_{\nu} \\
& \left.+\frac{1}{2} \frac{m^{2}}{n \cdot \partial} n_{v} \tilde{F}_{\mu}-\frac{1}{2} \frac{m^{2}}{n \cdot \partial} n_{\nu} \overline{\tilde{F}}_{\mu}\right) \\
& \overline{\tilde{\mathcal{C}}}_{\mu \nu}(x, \theta, \bar{\theta})=\overline{\tilde{c}}_{\mu \nu}(x)+\theta\left(\tilde{M}_{\mu \nu}-B_{\mu \nu}\right. \\
& \left.+\bar{B}_{\mu \nu}\right)+\bar{\theta} \tilde{c}_{\mu \nu}^{\star}+\theta \bar{\theta} \bar{m}_{\mu \nu}, \\
& \mathcal{B}_{\mu \nu}(x, \theta, \bar{\theta})=B_{\mu \nu}(x)+\theta N_{\mu \nu}+\bar{\theta}\left(B_{\mu \nu}^{\star}+\partial_{\mu} \tilde{f}_{\nu}\right. \\
& -\partial_{\mu} \overline{\tilde{f}}_{\nu}-\partial_{\nu} \tilde{f}_{\mu}+\partial_{\nu} \overline{\tilde{f}}_{\mu}-\frac{1}{2} \frac{m^{2}}{n \cdot \partial} n_{\mu} \tilde{f}_{\nu} \\
& +\frac{1}{2} \frac{m^{2}}{n \cdot \partial} n_{\mu} \overline{\tilde{f}}_{\nu}+\frac{1}{2} \frac{m^{2}}{n \cdot \partial} n_{\nu} \tilde{f}_{\mu} \\
& \left.-\frac{1}{2} \frac{m^{2}}{n \cdot \partial} n_{\nu} \overline{\tilde{f}}_{\mu}\right)+\theta \bar{\theta} n_{\mu \nu}, \\
& \overline{\mathcal{B}}_{\mu \nu}(x, \theta, \bar{\theta})=\bar{B}_{\mu \nu}(x)+\theta N_{\mu \nu}+\bar{\theta} B_{\mu \nu}^{\star}+\theta \bar{\theta} n_{\mu \nu}, \\
& \tilde{\mathcal{B}}_{\mu \nu}(x, \theta, \bar{\theta})=\tilde{B}_{\mu \nu}(x)+\theta \tilde{N}_{\mu \nu}+\bar{\theta} \tilde{B}_{\mu \nu}^{\star}+\theta \bar{\theta} \bar{n}_{\mu \nu}, \\
& \mathcal{B}_{\mu}(x, \theta, \bar{\theta})=\beta_{\mu}(x)+\theta O_{\mu}+\bar{\theta}\left(\beta_{\mu}^{\star}+F_{\mu}-\bar{F}_{\mu}\right) \\
& +\theta \bar{\theta}\left(o_{\mu}-\partial_{\mu} B_{2}+\partial_{\mu} \bar{B}_{2}+\frac{1}{2} \frac{m^{2}}{n \cdot \partial} n_{\mu} B_{2}\right. \\
& \left.-\frac{1}{2} \frac{m^{2}}{n \cdot \partial} n_{\mu} \bar{B}_{2}\right) \\
& \overline{\tilde{\mathcal{B}}}_{\mu \nu}(x, \theta, \bar{\theta})=\overline{\tilde{B}}_{\mu \nu}(x)+\theta\left(\tilde{N}_{\mu \nu}-\left(\partial_{\mu} f_{\nu}-\partial_{\mu} \bar{f}_{\nu}\right.\right. \\
& -\partial_{\nu} f_{\mu}+\partial_{\nu} \bar{f}_{\mu}-\frac{1}{2} \frac{m^{2}}{n \cdot \partial} n_{\mu} f_{\nu}+\frac{1}{2} \frac{m^{2}}{n \cdot \partial} n_{\mu} \bar{f}_{\nu} \\
& \begin{array}{c}
\overline{\mathcal{B}}_{\mu}(x, \theta, \bar{\theta})=\bar{\beta}_{\mu}(x)+\theta\left(O_{\mu}-\partial_{\mu} c_{2}+\partial_{\mu} \bar{c}_{2}\right. \\
\left.+\frac{1}{2} \frac{m^{2}}{n \cdot \partial} n_{\mu} c_{2}-\frac{1}{2} \frac{m^{2}}{n \cdot \partial} n_{\mu} \bar{c}_{2}\right)+\bar{\theta} \beta_{\mu}^{\star}+\theta \bar{\theta} o_{\mu},
\end{array} \\
& \tilde{\mathcal{B}}_{\mu}(x, \theta, \bar{\theta})=\tilde{\beta}_{\mu}(x)+\theta \tilde{O}_{\mu}+\bar{\theta}\left(\tilde{\beta}_{\mu}^{\star}+\partial_{\mu} \tilde{c}_{2}-\partial_{\mu} \overline{\tilde{c}}_{2}\right. \\
& \left.-\frac{1}{2} \frac{m^{2}}{n \cdot \partial} n_{\mu} \tilde{c}_{2}+\frac{1}{2} \frac{m^{2}}{n \cdot \partial} n_{\mu} \overline{\tilde{c}}_{2}\right) \\
& +\theta \bar{\theta}\left(\bar{o}_{\mu}+\partial_{\mu} B_{2}-\partial_{\mu} \bar{B}_{2}-\frac{1}{2} \frac{m^{2}}{n \cdot \partial} n_{\mu} B_{2}+\frac{1}{2} \frac{m^{2}}{n \cdot \partial} n_{\mu} \bar{B}_{2}\right), \\
& \overline{\tilde{\mathcal{B}}}_{\mu}(x, \theta, \bar{\theta})=\overline{\tilde{\beta}}_{\mu}(x)+\theta\left(\tilde{O}_{\mu}-\tilde{F}_{\mu}+\overline{\tilde{F}}_{\mu}\right)+\bar{\theta} \tilde{\beta}_{\mu}^{\star}+\theta \bar{\theta} \bar{o}_{\mu}, \\
& \mathcal{F}_{\mu}(x, \theta, \bar{\theta})=F_{\mu}(x)+\theta P_{\mu}+\bar{\theta} F_{\mu}^{\star}+\theta \bar{\theta} p_{\mu}, \\
& \overline{\mathcal{F}}_{\mu}(x, \theta, \bar{\theta})=\bar{F}_{\mu}(x)+\theta\left(P_{\mu}+\partial_{\mu} B-\partial_{\mu} \bar{B}\right. \\
& \left.-\frac{1}{2} \frac{m^{2}}{n \cdot \partial} n_{\mu} B+\frac{1}{2} \frac{m^{2}}{n \cdot \partial} n_{\mu} \bar{B}\right)+\bar{\theta} F_{\mu}^{\star}+\theta \bar{\theta} p_{\mu}, \\
& \tilde{\mathcal{F}}_{\mu}(x, \theta, \bar{\theta})=\tilde{F}_{\mu}(x)+\theta \tilde{P}_{\mu}+\bar{\theta}\left(\tilde{F}_{\mu}^{\star}-\partial_{\mu} B_{2}+\partial_{\mu} \bar{B}_{2}\right. \\
& \left.+\frac{1}{2} \frac{m^{2}}{n \cdot \partial} n_{\mu} B_{2}-\frac{1}{2} \frac{m^{2}}{n \cdot \partial} n_{\mu} \bar{B}_{2}\right)+\theta \bar{\theta} \bar{p}_{\mu}, \\
& \overline{\tilde{\mathcal{F}}}_{\mu}(x, \theta, \bar{\theta})=\overline{\tilde{F}}_{\mu}(x)+\theta \tilde{P}_{\mu}+\bar{\theta} \tilde{F}_{\mu}^{\star}+\theta \bar{\theta} \bar{p}_{\mu}, \\
& \mathfrak{F}_{\mu}(x, \theta, \bar{\theta})=f_{\mu}(x)+\theta Q_{\mu}+\bar{\theta}\left(f_{\mu}^{\star}-\partial_{\mu} B_{1}+\partial_{\mu} \bar{B}_{1}\right. \\
& \left.+\frac{1}{2} \frac{m^{2}}{n \cdot \partial} n_{\mu} B_{1}-\frac{1}{2} \frac{m^{2}}{n \cdot \partial} n_{\mu} \bar{B}_{1}\right)+\theta \bar{\theta} q_{\mu}, \\
& \overline{\mathfrak{F}}_{\mu}(x, \theta, \bar{\theta})=\bar{f}_{\mu}(x)+\theta Q_{\mu}+\bar{\theta} f_{\mu}^{\star}+\theta \bar{\theta} q_{\mu}, \\
& \tilde{\mathfrak{F}}_{\mu}(x, \theta, \bar{\theta})=\tilde{f}_{\mu}(x)+\theta \tilde{Q}_{\mu}+\bar{\theta} \tilde{f}_{\mu}^{\star}+\theta \bar{\theta} \bar{q}_{\mu}, \\
& \overline{\tilde{\mathfrak{F}}}_{\mu}(x, \theta, \bar{\theta})=\overline{\tilde{f}}_{\mu}(x)+\theta\left(\tilde{Q}_{\mu}-\partial_{\mu} B_{1}+\partial_{\mu} \bar{B}_{1}\right. \\
& \left.+\frac{1}{2} \frac{m^{2}}{n \cdot \partial} n_{\mu} B_{1}-\frac{1}{2} \frac{m^{2}}{n \cdot \partial} n_{\mu} \bar{B}_{1}\right)+\bar{\theta} \tilde{f}_{\mu}^{\star}+\theta \bar{\theta} \bar{q}_{\mu}, \\
& \mathcal{C}_{2}(x, \theta, \bar{\theta})=c_{2}(x)+\theta \Re+\bar{\theta}\left(c_{2}^{\star}+B-\bar{B}\right)+\theta \bar{\theta} r, \\
& \overline{\mathcal{C}}_{2}(x, \theta, \bar{\theta})=\bar{c}_{2}(x)+\theta \Re+\bar{\theta} c_{2}^{\star}+\theta \bar{\theta} r, \\
& \tilde{\mathcal{C}}_{2}(x, \theta, \bar{\theta})=\tilde{c}_{2}(x)+\theta \tilde{\Re}+\bar{\theta} \tilde{c}_{2}^{\star}+\theta \bar{\theta} \bar{r}, \\
& \overline{\tilde{\mathcal{C}}}_{2}(x, \theta, \bar{\theta})=\overline{\tilde{c}}_{2}(x)+\theta\left(\tilde{\Re}-B_{2}+\bar{B}_{2}\right)+\bar{\theta} \tilde{c}_{2}^{\star}+\theta \bar{\theta} \bar{r}, \\
& \mathcal{C}_{1}(x, \theta, \bar{\theta})=c_{1}(x)+\theta \mathfrak{S}+\bar{\theta}\left(c_{1}^{\star}-B_{1}+\bar{B}_{1}\right)+\theta \bar{\theta} s, \\
& \overline{\mathcal{C}}_{1}(x, \theta, \bar{\theta})=\bar{c}_{1}(x)+\theta(\mathfrak{S}+B-\bar{B})+\bar{\theta} c_{1}^{\star}+\theta \bar{\theta} s, \\
& \tilde{\mathcal{C}}_{1}(x, \theta, \bar{\theta})=\tilde{c}_{1}(x)+\theta \tilde{\mathfrak{S}}+\bar{\theta}\left(\tilde{c}_{1}^{\star}-B_{2}+\bar{B}_{2}\right)+\theta \bar{\theta} \bar{s}, \\
& \overline{\tilde{\mathcal{C}}}_{1}(x, \theta, \bar{\theta})=\overline{\tilde{c}}_{1}(x)+\theta\left(\tilde{\mathfrak{S}}-B_{1}+\bar{B}_{1}\right)+\bar{\theta} \tilde{c}_{1}^{\star}+\theta \bar{\theta} \bar{s}, \\
& \Phi_{\mu}(x, \theta, \bar{\theta})=\phi_{\mu}(x)+\theta T_{\mu}+\bar{\theta}\left(\phi_{\mu}^{\star}+\tilde{f}_{\mu}-\overline{\tilde{f}}_{\mu}\right) \\
& +\theta \bar{\theta}\left(t_{\mu}+\partial_{\mu} B_{1}-\partial_{\mu} \bar{B}_{1}-\frac{1}{2} \frac{m^{2}}{n \cdot \partial} n_{\mu} B_{1}\right. \\
& \left.+\frac{1}{2} \frac{m^{2}}{n \cdot \partial} n_{\mu} \bar{B}_{1}\right)
\end{aligned}
$$




$$
\begin{aligned}
& \bar{\Phi}_{\mu}(x, \theta, \bar{\theta})=\bar{\phi}_{\mu}(x)+\theta\left(T_{\mu}-f_{\mu}+\bar{f}_{\mu}\right)+\bar{\theta} \phi_{\mu}^{\star}+\theta \bar{\theta} t_{\mu}, \\
& \mathcal{B}(x, \theta, \bar{\theta})=B(x)+\theta \mathbb{U}+\bar{\theta} B^{\star}+\theta \bar{\theta} u \\
& \overline{\mathcal{B}}(x, \theta, \bar{\theta})=\bar{B}(x)+\theta \mathbb{U}+\bar{\theta} B^{\star}+\theta \bar{\theta} u, \\
& \mathcal{B}_{1}(x, \theta, \bar{\theta})=B_{1}(x)+\theta \mathfrak{V}+\bar{\theta} B_{1}^{\star}+\theta \bar{\theta} v, \\
& \overline{\mathcal{B}}_{1}(x, \theta, \bar{\theta})=\bar{B}_{1}(x)+\theta \mathfrak{V}+\bar{\theta} B_{1}^{\star}+\theta \bar{\theta} v, \\
& \mathcal{B}_{2}(x, \theta, \bar{\theta})=B_{2}(x)+\theta \mathfrak{W}+\bar{\theta} B_{2}^{\star}+\theta \bar{\theta} w, \\
& \overline{\mathcal{B}}_{2}(x, \theta, \bar{\theta})=\bar{B}_{2}(x)+\theta \mathfrak{W}+\bar{\theta} B_{2}^{\star}+\theta \bar{\theta} w .
\end{aligned}
$$

Form the above relations, we calculate

$$
\begin{aligned}
& \frac{1}{2} \frac{\delta}{\delta \bar{\theta}} \frac{\delta}{\delta \theta} \overline{\mathcal{B}}_{\mu \nu \eta} \overline{\mathcal{B}}^{\mu \nu \eta}=l_{\mu \nu \eta} \bar{B}^{\mu \nu \eta}-B_{\mu \nu \eta}^{\star} \\
& \times\left(L^{\mu \nu \eta}-\partial^{\mu} c^{\nu \eta}+\partial^{\mu} \bar{c}^{\nu \eta}\right. \\
& -\partial^{\nu} c^{\eta \mu}+\partial^{\nu} \bar{c}^{\eta \mu}-\partial^{\eta} c^{\mu \nu}+\partial^{\eta} \bar{c}^{\mu \nu} \\
& +\frac{1}{2} \frac{m^{2}}{n \cdot \partial} n^{\mu} c^{\nu \eta}-\frac{1}{2} \frac{m^{2}}{n \cdot \partial} n^{\mu} \bar{c}^{\nu \eta} \\
& +\frac{1}{2} \frac{m^{2}}{n \cdot \partial} n^{\nu} c^{\eta \mu}+\frac{1}{2} \frac{m^{2}}{n \cdot \partial} n^{\nu} \bar{c}^{\eta \mu}+\frac{1}{2} \frac{m^{2}}{n \cdot \partial} n^{\eta} c^{\mu \nu} \\
& \left.-\frac{1}{2} \frac{m^{2}}{n \cdot \partial} n^{\eta} \bar{c}^{\mu \nu}\right) \text {, } \\
& \frac{\delta}{\delta \bar{\theta}} \frac{\delta}{\delta \theta} \overline{\tilde{\mathcal{C}}}_{\mu \nu} \overline{\mathcal{C}}^{\mu \nu}=\bar{m}_{\mu \nu} \bar{c}^{\mu \nu}+m_{\mu \nu} \overline{\tilde{c}}^{\mu \nu}+\tilde{c}_{\mu \nu}^{\star}\left(M^{\mu \nu}-\partial^{\mu} \beta^{\nu}\right. \\
& +\partial^{\mu} \bar{\beta}^{\nu}+\partial^{v} \beta^{\mu}-\partial^{v} \bar{\beta}^{\mu}+\frac{1}{2} \frac{m^{2}}{n \cdot \partial} n^{\mu} \beta^{v} \\
& -\frac{1}{2} \frac{m^{2}}{n \cdot \partial} n^{\mu} \bar{\beta}^{\nu}-\frac{1}{2} \frac{m^{2}}{n \cdot \partial} n^{\nu} \beta^{\mu} \\
& \left.+\frac{1}{2} \frac{m^{2}}{n \cdot \partial} n^{v} \bar{\beta}^{\mu}\right)+c_{\mu \nu}^{\star}\left(\tilde{M}^{\mu \nu}-B^{\mu \nu}+\bar{B}^{\mu \nu}\right), \\
& \frac{1}{2} \frac{\delta}{\delta \bar{\theta}} \frac{\delta}{\delta \theta} \overline{\tilde{\mathcal{B}}}_{\mu \nu} \overline{\tilde{\mathcal{B}}}^{\mu \nu}=n_{\mu \nu} \bar{B}^{\mu \nu}-B_{\mu \nu}^{\star} N^{\mu \nu}, \\
& \frac{1}{2} \frac{\delta}{\delta \bar{\theta}} \frac{\delta}{\delta \theta} \overline{\mathcal{B}}_{\mu \nu} \overline{\mathcal{B}}^{\mu \nu}=\bar{n}_{\mu \nu} \overline{\tilde{B}}^{\mu \nu}-\tilde{B}_{\mu \nu}^{\star}\left(\tilde{N}^{\mu \nu}-\partial^{\mu} f^{\nu}\right. \\
& +\partial^{\mu} \bar{f}^{v}+\partial^{v} f^{\mu}-\partial^{v} \bar{f}^{\mu}+\frac{1}{2} \frac{m^{2}}{n \cdot \partial} n^{\mu} f^{v} \\
& \left.-\frac{1}{2} \frac{m^{2}}{n \cdot \partial} n^{\mu} \bar{f}^{\nu}-\frac{1}{2} \frac{m^{2}}{n \cdot \partial} n^{\nu} f^{\mu}+\frac{1}{2} \frac{m^{2}}{n \cdot \partial} n^{\nu} \bar{f}^{\mu}\right), \\
& \frac{1}{2} \frac{\delta}{\delta \bar{\theta}} \frac{\delta}{\delta \theta} \overline{\mathcal{B}}_{\mu} \overline{\mathcal{B}}^{\mu}=o_{\mu} \bar{\beta}^{\mu}-\beta_{\mu}^{\star}\left(O^{\mu}-\partial^{\mu} c_{2}\right. \\
& \left.+\partial^{\mu} \bar{c}_{2}-\frac{1}{2} \frac{m^{2}}{n \cdot \partial} n^{\mu} c_{2}-\frac{1}{2} \frac{m^{2}}{n \cdot \partial} n^{\mu} \bar{c}_{2}\right), \\
& \frac{1}{2} \frac{\delta}{\delta \bar{\theta}} \frac{\delta}{\delta \theta} \overline{\tilde{\mathcal{B}}}_{\mu} \overline{\tilde{\mathcal{B}}}^{\mu}=\bar{o}_{\mu} \overline{\tilde{\beta}}^{\mu}-\tilde{\beta}_{\mu}^{\star}\left(\tilde{O}^{\mu}-\tilde{F}^{\mu}+\overline{\tilde{F}}^{\mu}\right), \\
& \frac{\delta}{\delta \bar{\theta}} \frac{\delta}{\delta \theta} \overline{\tilde{\mathcal{F}}}_{\mu} \overline{\mathcal{F}}^{\mu}=\bar{p}_{\mu} \bar{F}^{\mu}+p_{\mu} \overline{\tilde{F}}^{\mu}+\tilde{F}_{\mu}^{\star}\left(P^{\mu}+\partial^{\mu} B\right. \\
& \left.-\partial^{\mu} \bar{B}-\frac{1}{2} \frac{m^{2}}{n \cdot \partial} n^{\mu} B+\frac{1}{2} \frac{m^{2}}{n \cdot \partial} n^{\mu} \bar{B}\right)+F_{\mu}^{\star} \tilde{P}^{\mu}, \\
& \frac{\delta}{\delta \bar{\theta}} \frac{\delta}{\delta \theta} \overline{\tilde{\mathfrak{F}}}_{\mu} \overline{\mathfrak{F}}^{\mu}=\bar{q}_{\mu} \bar{f}^{\mu}+q_{\mu} \overline{\tilde{f}}^{\mu}+\tilde{f}_{\mu}^{\star} Q^{\mu}+f_{\mu}^{\star}\left(\tilde{Q}^{\mu}-\partial^{\mu} B_{1}\right. \\
& \left.+\partial^{\mu} \bar{B}_{1}+\frac{1}{2} \frac{m^{2}}{n \cdot \partial} n^{\mu} B_{1}-\frac{1}{2} \frac{m^{2}}{n \cdot \partial} n^{\mu} \bar{B}_{1}\right),
\end{aligned}
$$

$$
\begin{aligned}
& \frac{\delta}{\delta \bar{\theta}} \frac{\delta}{\delta \theta} \overline{\tilde{C}}_{2} \overline{\mathcal{C}}_{2}=\bar{r}_{\bar{c}_{2}}+r \overline{\tilde{c}}_{2}+\tilde{c}_{2}^{\star} \mathfrak{R}+c_{2}^{\star}\left(\tilde{\mathfrak{R}}-B_{2}+\bar{B}_{2}\right), \\
& \frac{\delta}{\delta \bar{\theta}} \frac{\delta}{\delta \theta} \overline{\tilde{\mathcal{C}}}_{1} \overline{\mathcal{C}}_{1}=\bar{s} \bar{c}_{1}+\tilde{c}_{1}^{\star}(\mathfrak{S}+B-\bar{B})+s \overline{\tilde{c}}_{1}+c_{1}^{\star}\left(\tilde{\mathfrak{S}}-B_{1}+\bar{B}_{1}\right), \\
& \frac{1}{2} \frac{\delta}{\delta \bar{\theta}} \frac{\delta}{\delta \theta} \bar{\Phi}_{\mu} \bar{\Phi}^{\mu}=t_{\mu} \bar{\phi}^{\mu}-\phi_{\mu}^{\star}\left(T^{\mu}-f^{\mu}+\bar{f}^{\mu}\right), \\
& \frac{1}{2} \frac{\delta}{\delta \bar{\theta}} \frac{\delta}{\delta \theta} \overline{\mathcal{B}} \overline{\mathcal{B}}=u \bar{B}-B^{\star} \mathbb{U}, \\
& \frac{1}{2} \frac{\delta}{\delta \theta} \frac{\delta}{\delta \theta} \overline{\mathcal{B}}_{1} \overline{\mathcal{B}}_{1}=v \bar{B}_{1}-B_{1}^{\star} \mathfrak{V}, \\
& \frac{1}{2} \frac{\delta}{\delta \bar{\theta}} \frac{\delta}{\delta \theta} \overline{\mathcal{B}}_{2} \overline{\mathcal{B}}_{2}=w \bar{B}_{2}-B_{2}^{\star} \mathfrak{W} .
\end{aligned}
$$

\section{References}

1. D. Colladay, V.A. Kostelecky, CPT violation and the standard model. Phys. Rev. D 55, 6760 (1997). arXiv:hep-ph/9703464

2. A.G. Cohen, S.L. Glashow, Very special relativity. Phys. Rev. Lett. 97, 021601 (2006). arXiv:hep-ph/0601236

3. A.G. Cohen, S.L. Glashow, A Lorentz-violating origin of neutrino mass?. arXiv:hep-ph/0605036

4. J. Fan, W.D. Goldberger, W. Skiba, Spin dependent masses and $\operatorname{Sim}(2)$ symmetry. Phys. Lett. B 649, 186 (2007). arXiv:hep-ph/0611049

5. A. Dunn, T. Mehen, Implications of $S U(2)_{L} \times U(1)$ symmetry for $\operatorname{SIM}(2)$ invariant neutrino masses, JLAB-THY-06-557. arXiv:hep-ph/0610202

6. E. Alvarez, R. Vidal, Very special (de Sitter) relativity. Phys. Rev. D 77, 127702 (2008). arXiv:0803.1949 [hep-th]

7. S. Upadhyay, P.K. Panigrahi, Quantum gauge freedom in very special relativity. Nucl. Phys. B 915, 168 (2017). arXiv:1608.03947 [hep-th]

8. S. Upadhyay, Reducible gauge theories in very special relativity. Eur. Phys. J. C 75, 593 (2015). arXiv: 1511.01063 [hep-th]

9. D.V. Ahluwalia, S.P. Horvath, Very special relativity as relativity of dark matter: the Elko connection. JHEP 1011, 078 (2010). arXiv:1008.0436 [hep-ph]

10. W. Mueck, Very special relativity in curved space-times. Phys. Lett. B 670, 95 (2008). arXiv:0806.0737 [hep-th]

11. M.M. Sheikh-Jabbari, A. Tureanu, A realization of the CohenGlashow very special relativity. Phys. Rev. Lett. 101, 261601 (2008). arXiv:0806.3699 [hep-th]

12. S. Das, S. Ghosh, S. Mignemi, Noncommutative spacetime in very special relativity. Phys. Lett. A 375, 3237 (2011). arXiv: 1004.5356 [hep-th]

13. A.G. Cohen, D.Z. Freedman, $\operatorname{Sim}(2)$ and SUSY. JHEP 07, 039 (2007). arXiv:hep-th/0605172

14. J. Vohanka, Gauge theory and $\operatorname{SIM}(2)$ superspace. Phys. Rev. D 85, 105009 (2012). arXiv:1112.1797 [hep-th]

15. G.W. Gibbons, J. Gomis, C.N. Pope, General very special relativity is Finsler geometry. Phys. Rev. D 76, 081701 (2007). arXiv:0707.2174 [hep-th]

16. A. Ilderton, Very special relativity as a background field theory. Phys. Rev. D 94, 045019 (2016). arXiv:1605.04967 [hep-th]

17. J. Alfaro, V.O. Rivelles, Very special relativity and Lorentz violating theories. Phys. Lett. B 734, 239 (2014). arXiv:1306.1941 [hep-th]

18. S. Cheon, C. Lee, S. Lee, SIM(2)-invariant modifications of electrodynamic theory. Phys. Lett. B 679, 73 (2009). arXiv:0904.2065 [hep-th]

19. J. Alfaro, V.O. Rivelles, Non-Abelian fields in very special relativity. Phys. Rev. D 88, 085023 (2013). arXiv:1305.1577 [hep-th] 
20. J. Wess, B. Zumino, Supergauge invariant extension of quantum electrodynamics. Nucl. Phys. B 78, 1 (1974)

21. J. Wess, Supersymmetry-Supergravity, Topics in Quantum Field Theory and Gauge Theories, Salamanca, 1977 (Springer, Bedim, 1978)

22. E. Cremmer, B. Julia, J. Scherk, Supergravity in theory in 11 dimensions. Phys. Lett. B 76, 409 (1978)

23. E. Cremmer, S. Ferrara, Formulation of 11-dimensional supergravity in superspace. Phys. Lett. B 91, 61 (1980)

24. L. Brink, P. Howe, Eleven-dimensional supergravity on the mass shell in superspace. Phys. Lett. B 91, 384 (1980)

25. M.B. Green, J.H. Schwarz, E. Witten, Superstring Theory (Cambridge University Press, Cambridge, 1987)

26. J. Polchinski, String Theory (Cambridge University Press, Cambridge, 1998)

27. M. Kalb, P. Ramond, Classical direct interstring action. Phys. Rev. D 9, 2273 (1974)

28. F. Lund, T. Regge, Unified approach to strings and vortices with soliton solutions. Phys. Rev. D 14, 1524 (1976)

29. M. Sato, S. Yahikozawa, "Topological" formulation of effective vortex strings. Nucl. Phys. B 436, 100 (1995). arXiv:hep-th/9406208

30. A. Sugamoto, Dual transformation in Abelian gauge theories. Phys. Rev. D 19, 1820 (1979)

31. R.L. Davis, E.P.S. Shellard, Antisymmetric tensors and spontaneous symmetry breaking. Phys. Lett. B 214, 219 (1988)

32. A. Salam, E. Sezgin, Supergravities in Diverse Dimensions (World Scientific, North-Hplland, 1989)

33. M. Henneaux, C. Teitelboim, Quantization of Gauge Systems (Princeton University Press, Princeton, 1992)

34. S. Weinberg, The Quantum Theory of Fields, Vol-II: Modern Applications (UK University Press, Cambridge, 1996)

35. I.A. Batalin, G.A. Vilkovisky, Gauge algebra and quantization. Phys. Lett. B 102, 27 (1981)

36. I.A. Batalin, G.A. Vilkovisky, Quantization of gauge theories with linearly dependent generators. Phys. Rev. D 28, 2567 (1983) [Erratum ibid D 30, 508 (1984)]

37. I.A. Batalin, G.A. Vilkovisky, Feynman rules for reducible gauge theories. Phys. Lett. B 120, 166 (1983)
38. S. Upadhyay, B.P. Mandal, Relating Gribov-Zwanziger theory and Yang-Mills theory in Batalin-Vilkovisky formalism. AIP Conf. Proc. 1444, 213 (2012). arXiv:1112.0422 [hep-th]

39. S. Upadhyay, B.P. Mandal, Generalized BRST transformation in Abelian rank-2 antisymmetric tensor field theory. Mod. Phys. Lett. A 25, 3347 (2010). arXiv:1004.0330 [hep-th]

40. J. Alfaro, P.H. Damgaard, Origin of antifields in the BatalinVilkovisky Lagrangian formalism. Nucl. Phys. B 404, 751 (1993). arXiv: hep-th/9301103

41. F. De Jonghe, Schwinger-Dyson BRST symmetry and the Batalin-Vilkovisky Lagrangian quantisation of gauge theories with open or reducible gauge algebras. J. Math. Phys. 35, 2734 (1994). arXiv: hep-th/9304025

42. W. Troost, P. van Nieuwenhuizen, A. Van Proeyen, Anomalies and the Batalin-Vilkovisky Lagrangian formalism. Nucl. Phys. B 333, $727(1990)$

43. J. Alfaro, P.H. Damgaard, Schwinger-Dyson equations as supersymmetric ward identities. Phys. Lett. B 222, 425 (1989)

44. J. Alfaro, P.H. Damgaard, J.I. Latorre, D. Montano, On the BRST invariance of field deformations. Phys. Lett. B 233, 153 (1989)

45. J. Alfaro, P.H. Damgaard, Origin of antifields in the BatalinVilkovisky Lagrangian formalism. Nucl. Phys. B 404, 751 (1993). arXiv:hep-th/9301103

46. N.R.F. Braga, A. Das, A superspace formulation of the BV action. Nucl. Phys. B 442, 655 (1995)

47. S. Upadhyay, B.P. Mandal, BV formulation of higher form gauge theories in a superspace. Eur. Phys. J. C 72, 2059 (2012)

48. M. Faizal, M. Khan, A superspace formulation of the BV action for higher derivative theories. Eur. Phys. J. C 71, 1603 (2011). arXiv:1103.0221 [hep-th]

49. C.-Y. Lee, Quantum field theory with a preferred direction: the very special relativity framework. Phys. Rev. D 93, 045011 (2016). arXiv:1512.09175 [hep-th]

50. S. Ferrara, O. Piguet, M. Schweda, Some supersymmetric aspects of the supertransformation of Becchi, Rouet and Stora. Nucl. Phys. B 119, 493 (1977)

51. K. Fujikawa, On a superfield theoretical treatment of the HiggsKibble mechanism. Prog. Theor. Phys. 59, 2045 (1978) 